\title{
TFEB/Mitf links impaired nuclear import to autophagolysosomal dysfunction in C9-ALS
}

Kathleen M. Cunningham ${ }^{1}$, Ke Zhang ${ }^{2}$, Kai Ruan², Kirstin Maulding ${ }^{1}$, Mumine Senturk ${ }^{4}$, Jonathan Grima ${ }^{3,5}$, Hyun Sung ${ }^{2}$, Zhongyuan Zuo ${ }^{6}$, Helen Song ${ }^{2}$, Jeffrey D. Rothstein ${ }^{1,2,3,5}$, Hugo J. Bellen ${ }^{4,6,7,8,9}$, Thomas E. Lloyd 1,2,5*

1. Cellular and Molecular Medicine Program, School of Medicine, Johns Hopkins University, Baltimore, MD 21205, USA

2. Department of Neurology, School of Medicine, Johns Hopkins University, Baltimore, MD 21205, USA

3. Brain Science Institute, School of Medicine, Johns Hopkins University, Baltimore, MD 21205, USA

4. Program in Developmental Biology, Baylor College of Medicine (BCM), Houston, TX, 77030, USA

5. Solomon H. Snyder Department of Neuroscience, School of Medicine, Johns Hopkins University, Baltimore, MD 21205, USA

6. Department of Molecular and Human Genetics, BCM, Houston TX, 77030, USA

7. Department of Neuroscience, BCM, Houston TX, 77030, USA

8. Jan and Dan Duncan Neurological Research Institute, Texas Children's Hospital, Houston TX, 77030, USA

9. Howard Hughes Medical Institute, BCM, Houston, TX, 77030, USA

* Correspondence to:

Thomas E. Lloyd, MD, PhD

Associate Professor of Neurology and Neuroscience

855 N. Wolfe St, Rangos 290

Baltimore, MD USA 21205

+1-410-502-6851

\section{tlloyd4@jhmi.edu}




\section{Abstract}

Disrupted nucleocytoplasmic transport (NCT) has been implicated in neurodegenerative disease pathogenesis; however, the mechanisms by which impaired NCT causes

5 neurodegeneration remain unclear. In a Drosophila screen, we identified Ref(2)p/p62, a key regulator of autophagy, as a potent suppressor of neurodegeneration caused by the GGGGCC hexanucleotide repeat expansion (G4C2 HRE) in C9orf72 that causes amyotrophic lateral sclerosis (ALS) and frontotemporal dementia (FTD). We found that p62 is increased and forms ubiquitinated aggregates due to decreased autophagic cargo degradation.

10 Immunofluorescence and electron microscopy of Drosophila tissues demonstrate an accumulation of lysosome-like organelles that precedes neurodegeneration. These phenotypes are partially caused by cytoplasmic mislocalization of Mitf/TFEB, a key transcriptional regulator of autophagolysosomal function. Additionally, TFEB is mislocalized and downregulated in human cells expressing GGGGCC repeats and in C9-ALS patient motor

15 cortex. Our data suggest that the C9orf72-HRE impairs Mitf/TFEB nuclear import, thereby disrupting autophagy and exacerbating proteostasis defects in C9-ALS/FTD. 


\section{Introduction}

A GGGGCC (G4C2) hexanucleotide repeat expansion (HRE) in chromosome 9 open reading frame 72 (C9orf72) is the most common genetic cause of amyotrophic lateral sclerosis (ALS) and frontotemporal dementia (FTD), accounting for up to $40 \%$ of cases of familial ALS

5 (DeJesus-Hernandez et al., 2011; Renton et al., 2011). ALS and/or FTD caused by mutations in C9orf72 (C9-ALS/FTD) is inherited in an autosomal dominant manner, suggesting that the HRE causes disease through gain-of-function or haploinsufficiency (DeJesus-Hernandez et al., 2011; Ling, Polymenidou, \& Cleveland, 2013). Loss of C9orf72 function has been linked to disruption of autophagy and lysosome function, though neurodegeneration is not observed in C9orf72 knockout mice (Liu et al., 2016; Y. Shi et al., 2018; Webster et al., 2016), suggesting that C9-ALS/FTD is likely primarily caused by toxicity of the HRE. Furthermore, expression of G4C2 repeats causes neurotoxicity in Drosophila and cell culture models of C9-ALS (Goodman et al., 2019; Kramer et al., 2016; Tran et al., 2015). This toxicity has been proposed to occur through either G4C2 repeat RNA-mediated sequestration of RNA-binding

15 proteins or translation of the G4C2 repeats into dipeptide repeat proteins (DPRs) through noncanonical repeat-associated non-AUG translation (Donnelly et al., 2013; Goodman et al., 2019; Mori et al., 2013; Tran et al., 2015).

We previously conducted a Drosophila screen of genes that bound with moderate-tohigh affinity to G4C2 RNA and identified modulation of the nucleocytoplasmic transport (NCT) pathway as a potent modifier of G4C2 toxicity in both fly and iPS neuron models of C9-ALS (K. Zhang et al., 2015), and this finding has also been made by other groups (Freibaum et al., 2015; Jovicic et al., 2015). The mechanism by which the G4C2 HRE disrupts NCT remain unclear, but potential mechanisms include G4C2 RNA binding to the master NCT regulator RanGAP (K. Zhang et al., 2015), DPRs binding to the nuclear pore complex (Boeynaems et 25 al., 2016; K. Y. Shi et al., 2017; Y. J. Zhang et al., 2016), stress granules sequestering NCT factors (K. Zhang et al., 2018), or through cytoplasmic TDP-43-dependent dysregulation of 
karyopherin- $\alpha$ (Chou et al., 2018; Gasset-Rosa et al., 2019; Solomon et al., 2018). Recently, a role for NCT disruption in Huntington's disease and Alzheimer's disease has been proposed, indicating that NCT disruption may be a common mechanism in many neurodegenerative diseases (Eftekharzadeh et al., 2018; Gasset-Rosa et al., 2017; Grima et al., 2017). However,

5 the pathways affected by NCT disruption that cause neurodegeneration have not yet been elucidated.

In a Drosophila screen for modifiers of G4C2-mediated neurodegeneration (K. Zhang et al., 2015), we identified Ref(2)p, the Drosophila homolog of p62/SQSTM1 (Sequestosome 1). p62/SQSTM1 is a rare genetic cause of ALS/FTD (Cirulli et al., 2015; Le Ber et al., 2013;

10 Teyssou et al., 2013) and functions in macroautophagy (hereafter termed autophagy) along with other genes implicated in ALS/FTD (Evans \& Holzbaur, 2019; Lin, Mao, \& Bellen, 2017; Ramesh \& Pandey, 2017) such as tank-binding kinase 1 (TBK1), optineurin (OPTN1), ubiquilin 2 and 4 (UBQN2 and 4), valosin-containing protein (VCP), CHMP2B, VapB, and the C9ORF72 protein itself (O'Rourke et al., 2015; Sellier et al., 2016; Sullivan et al., 2016; Ugolino et al.,

15 2016; Webster et al., 2016; Yang et al., 2016). During autophagy, organelles and protein aggregates are degraded via polyubiquitination and targeting to a newly forming autophagosome, followed by degradation upon fusion with the lysosome (Lin et al., 2017).

Although autophagy and nucleocytoplasmic transport have both been implicated in neurodegeneration, it is unclear whether or how these two pathways interact in disease 20 pathogenesis (Gao, Almeida, \& Lopez-Gonzalez, 2017; Thomas, Alegre-Abarrategui, \& WadeMartins, 2013). Here, we show that expression of $30 \mathrm{G} 4 \mathrm{C} 2$ repeats is sufficient to disrupt autophagy in Drosophila, leading to an accumulation of p62 and ubiquitinated protein aggregates. We find that autophagolysosomal defects are caused by loss of nuclear localization of the transcription factor Mitf (the Drosophila homolog of TFEB), which regulates

25 transcription of genes involved in autophagolysosome biogenesis (Bouche et al., 2016; Palmieri et al., 2011; Sardiello et al., 2009; T. Zhang et al., 2015). Furthermore, suppressing 
this NCT defect is sufficient to rescue Mitf nuclear localization, restoring autophagy and

lysosome function and rescuing neurodegeneration. These findings suggest a pathogenic cascade in C9-ALS/FTD whereby NCT disruption causes a failure of autophagosome biogenesis and lysosome dysfunction that ultimately leads to neuronal death.

\section{Results}

\section{Ref(2)p/p62 knockdown suppresses G4C2-mediated neurodegeneration}

Expression of $30 \mathrm{G} 4 \mathrm{C} 2$ repeats (30R) in the eye using GMR-Gal4 results in progressive photoreceptor degeneration and visible ommatidial disruption by day 15 (Figure 1A)(Xu et al., $\operatorname{ref}(2) p^{R N A i}$ was among the strongest of 32 suppressors of G4C2-mediated eye degeneration (K. Zhang et al., 2015) (Figure 1A). ref(2)p is the Drosophila homolog of p62/SQSTM1, and this modifier is of particular interest because SQSTM1 mutations that cause loss of selective autophagy cause ALS/FTD (Cirulli et al., 2015; Goode et al., 2016; Le Ber et al., 2013), and

15 p62 aggregates are pathological features of both familial and sporadic ALS (Al-Sarraj et al., 2011; Cooper-Knock et al., 2012). Knockdown of ref(2)p suppresses eye degeneration, whereas overexpression of $\operatorname{ref}(2) p$ strongly enhances this phenotype (Figure 1A-B, Figure S1B). Similarly, coexpression of ref(2)p RNAi partially rescues the pupal lethality seen with 30R expression in motor neurons (Figure 1C). To determine whether ref(2)p knockdown is

20 able to suppress age-dependent neurodegeneration, we used an inducible, pan-neuronal driver (elavGS-Gal4) in which 30R-expression leads to a marked reduction in climbing ability after 7 days (Figure 1D). This climbing defect is suppressed with coexpression of ref(2)p RNAi suggesting that $r e f(2) p$ contributes to $\mathrm{G} 4 \mathrm{C} 2$-mediated neurotoxicity in the adult brain. ref(2)p RNAi coexpression reduces ref(2)p levels by about $80 \%$ of control and does not alter G4C2

25 RNA levels (Figure S1C), suggesting that ref(2)p acts downstream of G4C2 transcription. 
bioRxiv preprint doi: https://doi.org/10.1101/2020.06.26.173021; this version posted June 26, 2020. The copyright holder for this preprint (which was not certified by peer review) is the author/funder, who has granted bioRxiv a license to display the preprint in perpetuity. It is made available under aCC-BY 4.0 International license.

toxicity in Drosophila (Kwon et al., 2014; Mizielinska \& Isaacs, 2014), we next tested whether ref(2)p knockdown rescues poly-Glycine-Arginine(GR) repeat-mediated toxicity (Mizielinska \& Isaacs, 2014). As shown in Figure S1B, ref(2)p RNAi partially rescues the severe eye degeneration phenotype caused by poly(GR)36 expression. Together, these data indicate that 5 ref(2)p, the Drosophila orthologue of p62/SQSTM1, modulates G4C2-mediated neurodegeneration.

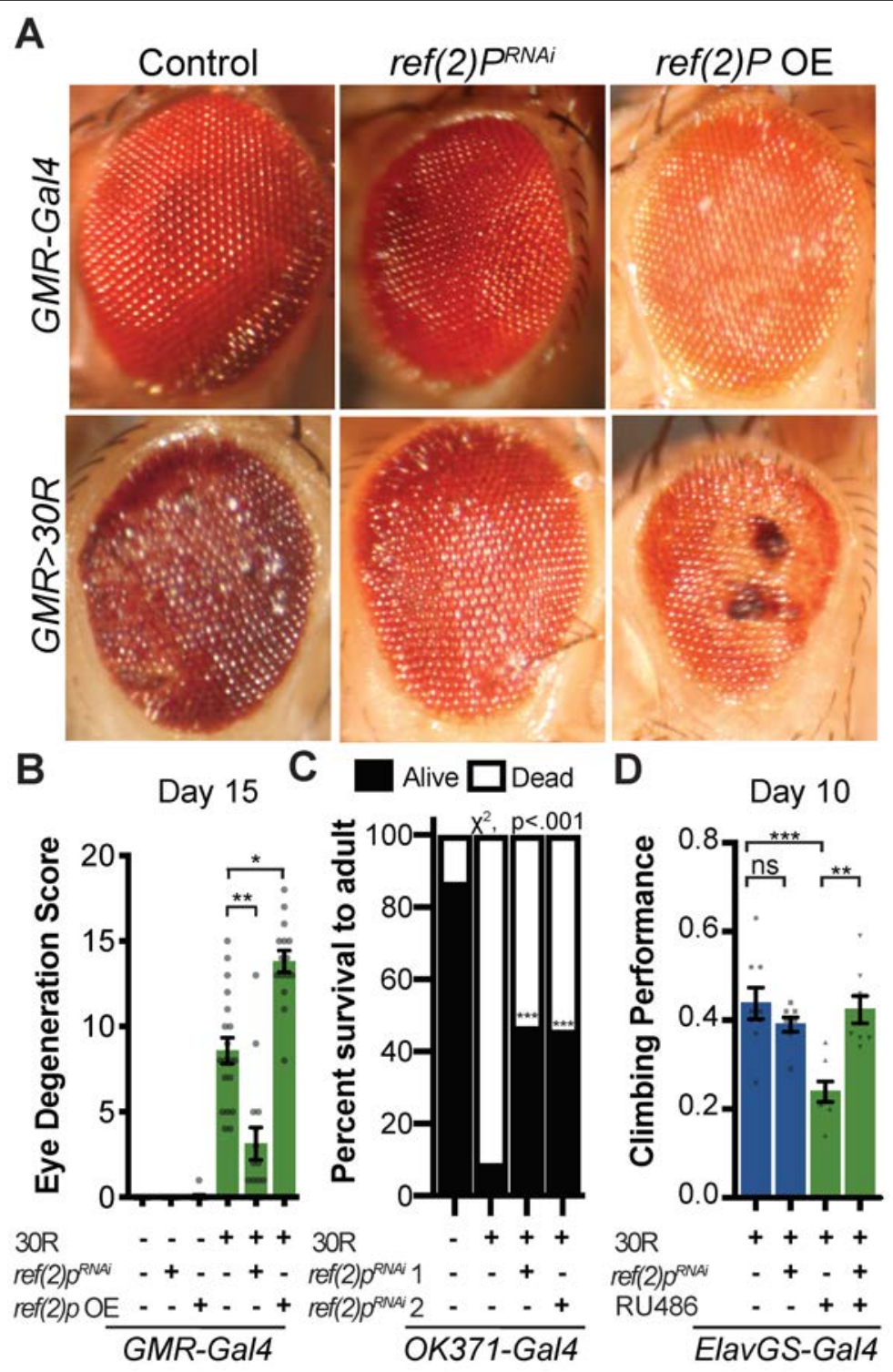

Figure 1: Autophagy receptor Ref(2)p / p62 genetically suppresses 30R-mediated degeneration. (A) 15-day old Drosophila eyes expressing GMR-Gal4 alone or GMR>30R (bottom row) with ref(2)p $p^{R N A i}$ or overexpression (OE) of HA-tagged ref(2)p. (B) Quantification of external eye degeneration, Kruskal-Wallis test, followed by Dunn's multiple comparisons, $n=15$. (C) Percent of pupal eclosion in flies expressing 30R under the control of the motor neuron OK371-Gal4 driver with OK371-Gal4 alone control, RNAi background control, or Ref(2)p RNAi $n \geq 100$ pupae, chi-squared test. (D) Adult flies expressing 30R under the control of the inducible, pan-neuronal elavGSGal4 induced with RU486 or vehicle and co-expressing control or ref(2) $p^{R N A i}$. $n=9,8,8,8$ groups of 10 flies. Oneway ANOVA with Bonferroni's Multiple Comparison's test. Data are mean \pm SEM. ${ }^{*}: p<.01{ }^{* *} p<.001{ }^{* * *} p<.0001$ 


\section{G4C2 repeat expression impairs autophagic flux}

p62/SQSTM1-positive inclusions are a common pathologic feature seen in brains of C9ALS/FTD patients where they colocalize with ubiquitin and DPRs (Al-Sarraj et al., 2011). We next investigated the localization of $\operatorname{Ref}(2) p$ protein (hereafter referred to as $p 62$ ) in motor

5 neurons. Expression of 30R leads to the formation of many large p62::GFP puncta in cell bodies compared to controls (Figure 2A-B, Figure S2A) that strongly colocalize with polyubiquitinated proteins (Figure 2B). Western blot analysis demonstrates that p62 protein is strongly upregulated in flies ubiquitously expressing 30R (Figure 2C, Figure S2C). Similarly, immunofluorescence staining with a p62 antibody shows endogenous p62 accumulations colocalizing with polyubiquitinated proteins in the ventral nerve cord, eye disc, and salivary gland of flies ubiquitously overexpressing 30R (Figure S2D). These data show that G4C2 repeat expression in fly models recapitulates the p62 protein accumulation with insoluble ubiquitinated protein aggregates seen in C9-ALS/FTD patient tissue and iPS neurons (Almeida et al., 2013; Mackenzie, Frick, \& Neumann, 2014). Increased p62 levels can be due to either increased transcription and/or translation or insufficient protein degradation (Korolchuk, Menzies, \& Rubinsztein, 2010). Using qRT-PCR, we show ref(2)p transcript levels are unchanged in G4C2 repeat-expressing larvae (Figure S1D), suggesting that G4C2 repeats cause p62 upregulation via inhibiting p62 degradation. Since p62 is degraded by autophagy and, indeed, disrupted autophagic flux is known to cause

20 p62 upregulation, we analyzed autophagy in G4C2-repeat-expressing flies. We first coexpressed the autophagosome marker mCherry::Atg8 (the fly orthologue of mammalian LC3) with $30 \mathrm{R}$ in fly motor neurons and found a marked reduction in mCherry::Atg8 vesicles when compared to wild-type controls (Figure 2D-E). p62 accumulation and loss of Atg8 puncta were recapitulated in multiple Drosophila models of C9-ALS/FTD (Figure S2E-H). Reduction of

25 Atg8-positive vesicles coupled with the accumulation of p62 and ubiquitin suggest that autophagic flux is impaired in G4C2-expressing animals. 

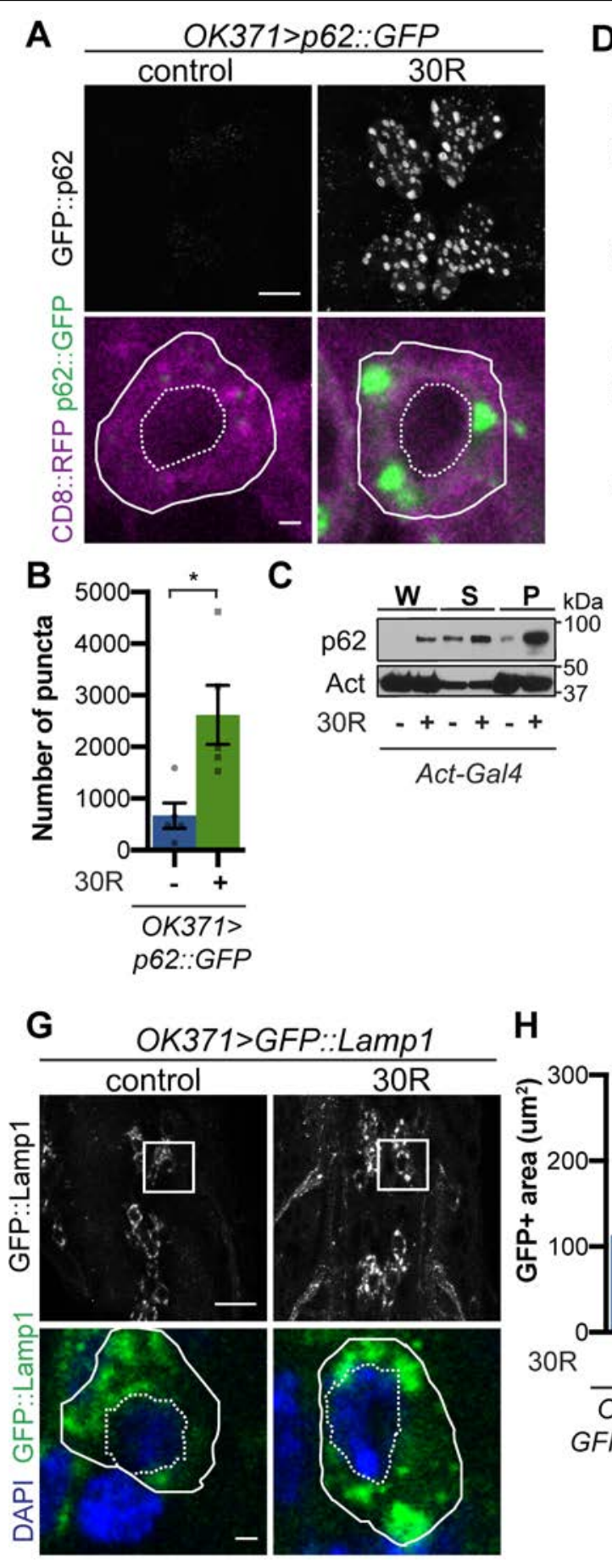

H
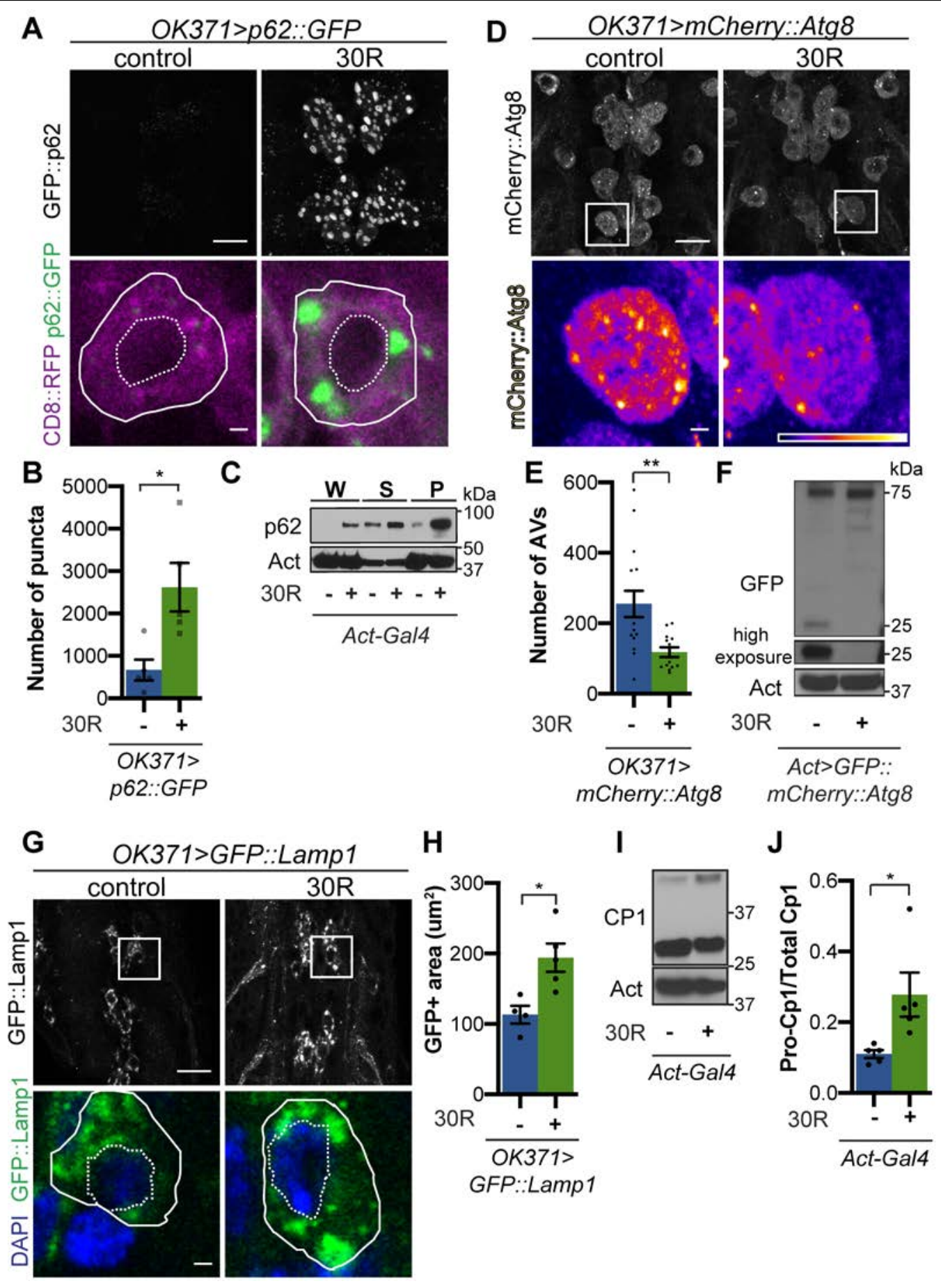

Figure 2: G4C2 repeat expression impairs autophagic flux. (A) Drosophila motor neurons expressing p62::GFP with or without 30R showing motor neuron cell bodies or a representative single cell body (below). (B)

Quantification of p62::GFP puncta in motor neuron cell bodies by number ( $n=5$ larvae, Mann-Whitney test), (C) Western blot of anti-p62 showing the whole (W), supernatant $(\mathrm{S})$ and pellet $(\mathrm{P})$ fractions of larvae ubiquitously expressing 30R under the control of Act-Gal4. (D) Larval motor neurons expressing mCherry::Atg8 with or without 30R showing cell bodies with representative single cell highlighting Atg8-positive puncta (below). (E) Quantification of Atg8-positive autophagic vesicles (AVs) in the ventral nerve cord of OK371-Gal4/+ $(n=16)$ or OK371>30R $(n=13$ larvae ,Student's t-test). (F) Anti-GFP Western of whole larvae ubiquitously expressing GFP::mCherry::Atg8 with or without 30R under the control of Act-Gal4 showing full length GFP::mCherry::Atg8 and cleaved GFP (25 kDa band). G) Larval motor neurons expressing GFP::Lamp (N-terminal (luminal) GFP) with or without 30R in cell bodies. (H) Quantification of GFP::Lamp positive area in (G), $n=5$ larvae, Student's t-test . (I) Western of whole larvae with or without 30R blotted for the lysosomal protease CP1, showing pro- (inactive, top) and cleaved (active, lower) bands. (J) Quantification of the ratio of pro- to cleaved band in $(\mathrm{I})(n=3){ }^{*}: p<0.05,{ }^{* *} p<.001$. Data are mean \pm SEM. 
bioRxiv preprint doi: https://doi.org/10.1101/2020.06.26.173021; this version posted June 26, 2020. The copyright holder for this preprint (which was not certified by peer review) is the author/funder, who has granted bioRxiv a license to display the preprint in perpetuity. It is made available under aCC-BY 4.0 International license.

\section{G4C2 repeat expression causes lysosome defects}

To investigate the autophagic pathway defects in G4C2 repeat-expressing neurons, we performed Transmission Electron Microscopy (TEM) on Drosophila eyes. As GMR-Gal4 is expressed throughout the development of photoreceptors (PRs), we chose to perform

5 electroretinograms (ERGs) of fly eyes selectively expressing $30 \mathrm{R}$ in photoreceptor neurons using $r h 1-G a l 4$, which turns on during adulthood. rh1>30R PRs show only a mild reduction of ON transient amplitude at 28 days, but complete loss of ON and OFF transients and decrease in ERG amplitude by 56 days (Figure S3A-D), indicating a loss in synaptic transmission and impaired phototransduction pathway respectively. These changes correspond to marked loss

10 of photoreceptors and synaptic terminals by 54 days which are not observed at 28 days

(Figure 3A-B; Figure S3E).

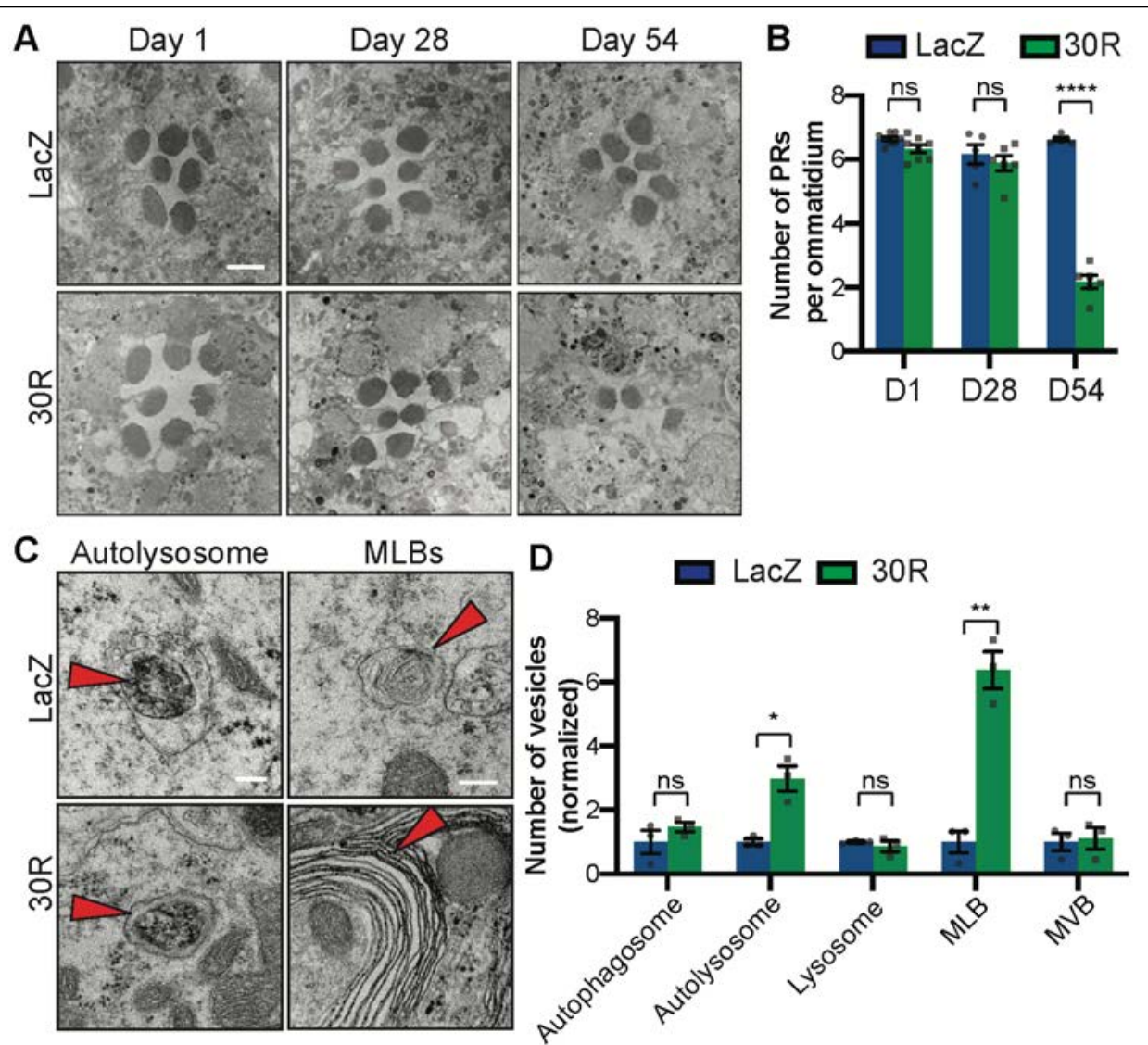

Figure 3. Autophago/lysosomal defects precede neurodegeneration in photoreceptor neurons. (A) TEM of rhabdomeres (cell bodies) in rhodopsin1-Gal4 driving LacZ (control) or 30R at Day 1, Day 28, and Day 54 after eclosion. B) Quantification of number of healthy (not split) photoreceptors per ommatidium in (A), $n=8,8,6,6,6,6$ flies, Student's t-test. (C) TEM images at 28 days of Drosophila eyes (rhabdomeres) showing representative autolysosomes and multilamellar bodies (MLBs), with or without 30R repeats expressed by rhodopsin1-Gal4. D) Quantification to the right of different vesicle types in TEM at day 28 (Student's t-test, $n=3$ adults). Data reported are mean \pm SEM. n.s., not significant ${ }^{*}, p<0.05,{ }^{* *}, p<0.01,{ }^{* * * *} p<.0001$. 
We therefore examined autophagic structures by TEM at 28 days, prior to cell loss.

Strikingly, we observe a marked increase in the size and number of multilamellar bodies

(MLBs) (Figure 3C-D). MLBs are commonly observed in lysosomal storage diseases and result from a deficiency of lysosomal hydrolases and accumulations of lysosomal lipids and

5 membranes (Hariri et al., 2000; Weaver, Na, \& Stahlman, 2002). Though we did not detect an alteration in the number of autophagosomes, lysosomes, or multivesicular bodies, we did see a significant increase in the number of autolysosomes (Figure 3C-D). These data suggest that autophagolysosomal function is disrupted in G4C2-expressing photoreceptor neurons at early stages of degeneration.

To further study lysosomal morphology and function, we expressed Lysosome Associated Membrane Protein (LAMP) with luminally-tagged GFP in our control and G4C2expressing flies. Since GFP is largely quenched by the acidity of lysosomes in control animals (Pulipparacharuvil et al., 2005), the accumulation of GFP::Lamp-positive vesicles in 30Rexpressing motor neurons suggests a defect in lysosomal acidity or targeting of GFP::Lamp to

15 mature lysosomes (Figure 2G-H). Furthermore, we observe a marked increase in size and number of late endosomes and lysosomes using genomically tagged Rab7::YFP throughout 30R-expressing motor neurons (Figure S4A) without alterations in early endosomes labeled with Rab5:YFP (data not shown). Together, these data demonstrate a marked expansion of the late endosome/lysosome compartment in G4C2-expressing neurons. Though accumulation of p62 and ubiquitinated proteins could be caused by a failure of autophagic vesicles to fuse with the degradative endolysosomal compartment, we did not detect a decrease in Atg8+, Rab7+ amphisomes in G4C2-expressing motor neuron cell bodies (Figure S4B-F). To assess autophagolysosomal function after fusion, we performed a "GFP liberation assay" on larvae expressing GFP::mCherry::Atg8 (Klionsky et al., 2016; Mauvezin, 25 Ayala, Braden, Kim, \& Neufeld, 2014). GFP is degraded more slowly than the rest of the Atg8 protein, leaving a population of free GFP in functioning lysosomes. Free lysosomal GFP is not 
observed in G4C2-expressing larvae, suggesting an impairment in GFP::mCherry::Atg8 degradation by the lysosome (Figure $2 \mathrm{~F}$ ). To directly probe lysosome enzymatic activity, we performed Western analysis of Drosophila cathepsin CP1. Whereas pro-CP1 is normally cleaved to its mature form by acid hydrolases in lysosomes (Kinser \& Dolph, 2012), larvae

5 ubiquitously expressing $30 \mathrm{R}$ show an increase in the ratio of pro-CP1 to CP1, indicating a decrease in pro-CP1 cleavage efficiency (Fig 2I-J). Together, these data suggest that lysosomes are expanded and dysfunctional in G4C2 repeat-expressing animals.

Given the impairment in autophagic flux, we hypothesized that genetic or pharmacologic manipulations that accelerate autophagy may suppress neurodegenerative phenotypes, whereas those that further impede autophagy would enhance the phenotypes. Indeed, in a candidate-based screen, activation of early steps in the autophagic pathway (e.g. by Atg1 overexpression) suppresses eye degeneration and blocking autophagosome/lysosome fusion (e.g. by Snap29 knockdown) enhances eye degeneration (Table S1). Similarly, pharmacologic activation of autophagy via inhibition of mTOR with rapamycin or mTor-independent activation

15 via trehalose rescues neurodegenerative phenotypes and p62 accumulation (Figure S4G-J). Together, these data show that promoting autophagy or lysosomal fusion are potent suppressors of G4C2-mediated neurodegeneration.

\section{Nucleocytoplasmic transport impairment disrupts autophagic flux} Isaacs, 2018; Evans \& Holzbaur, 2019; Gao et al., 2017; Lin et al., 2017; Ling et al., 2013; Ramesh \& Pandey, 2017). However, the sequence of events in the pathogenic cascade remains unknown. Cytoplasmic protein aggregates or RNA stress granule formation is 
We therefore tested whether defects in autophagy are upstream, downstream, or in parallel with defects in nucleocytoplasmic transport.

We first tested whether knockdown of $\operatorname{ref}(2) p$ rescues the mislocalization of the NCT reporter shuttle-GFP (S-GFP) containing both a nuclear localization sequence (NLS) and

5 nuclear export sequence (NES). G4C2 repeat expression causes mislocalization of S-GFP to the cytoplasm (K. Zhang et al., 2015), but knockdown of ref(2)p does not restore nuclear localization ( Figure S5A). Similarly, stimulation of autophagy with rapamycin or trehalose fails to rescue S-GFP mislocalization in G4C2 expressing salivary glands (Figure S5B). Restoring autophagy does not rescue NCT defects although it can rescue neurodegeneration, suggesting that autophagy defects are either independent of or downstream of NCT defects. Indeed, RanGAP knockdown is sufficient to increase the number and size of p62::GFP puncta, similar to the effects of overexpressing the G4C2 repeats (Figure S5C), suggesting that NCT disruption is sufficient to disrupt autophagic flux in Drosophila motor neurons.

\section{Mitf is mislocalized and inactivated in Drosophila models of C9-ALS/FTD}

Because we observed a reduction in autophagosomes and expansion of lysosomerelated organelles, we hypothesized that transcription factors regulating autophagolysosomal function may be mislocalized to the cytoplasm due to disrupted nuclear import. The TFE family of transcription factors (TFEB, TFE3, MITF, and TFEC) regulates multiple steps of autophagy

20 from autophagosome biogenesis through lysosome acidification via a network of genes called the Coordinated Lysosome Expression And Regulation (CLEAR) network (Settembre et al., 2011). These transcription factors are regulated by phosphorylation of canonical nuclear export (NES) and nuclear import (NLS) sequences (Li et al., 2018). In Drosophila, this conserved transcription factor family is represented by a single homolog called Mitf (Bouche et al., 2016;

25 T. Zhang et al., 2015). 

available under aCC-BY 4.0 International license.

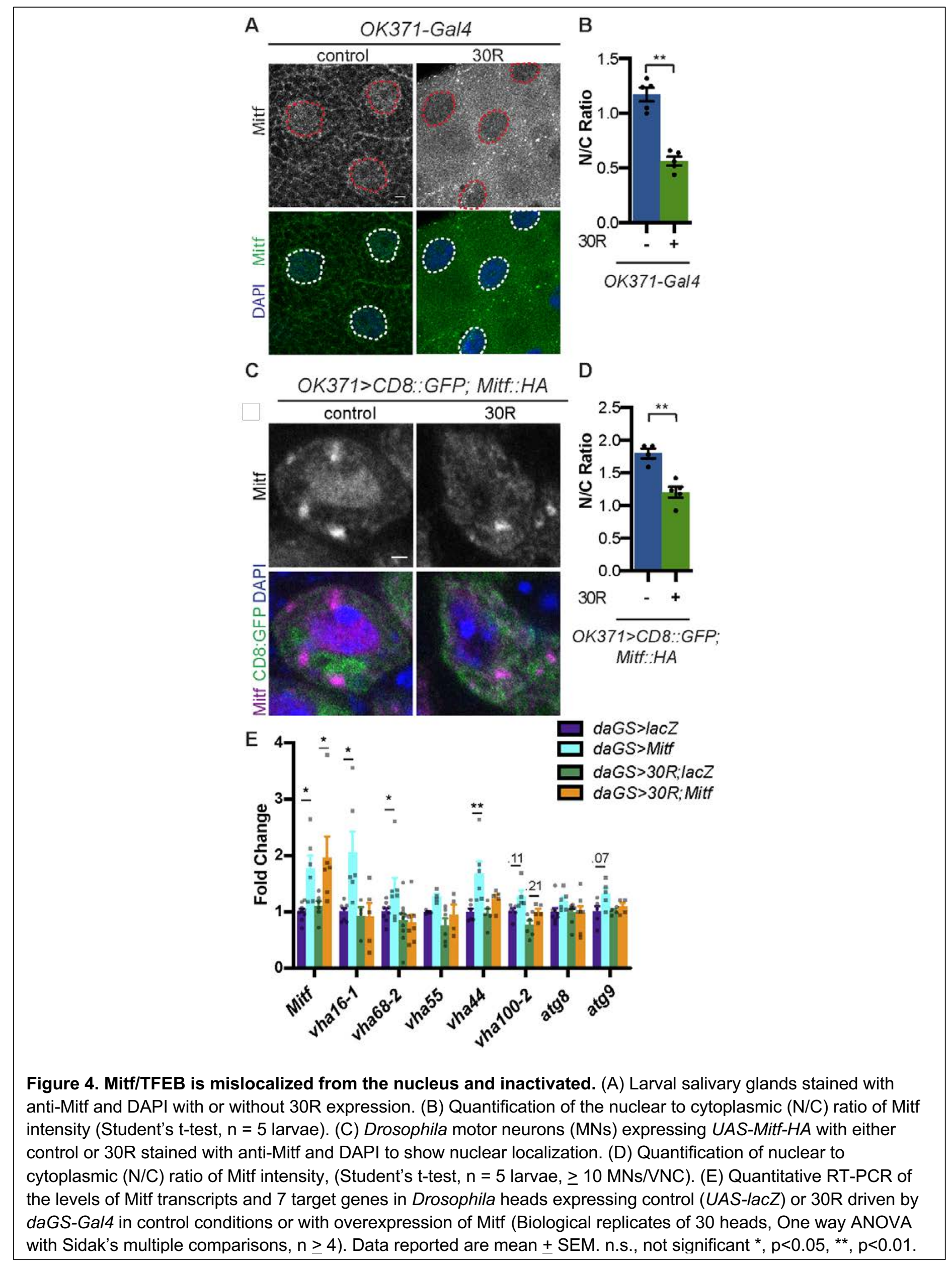


Mitf knockdown in the nervous system causes lysosomal defects similar to those we observe in G4C2-expressing flies (Bouche et al., 2016; Hallsson et al., 2004; Sardiello et al., 2009; Song et al., 2013). Additionally, TFEB levels are reduced in SOD1 cell culture and mouse ALS models (Chen et al., 2015) as well as in ALS and Alzheimer's patient brain tissue

5 (Wang, Wang, Xu, \& Lakshmana, 2016). Therefore, we hypothesized that impaired Mitf nuclear import might underlie the autophagolysosomal phenotypes in fly models of C9-ALS. Indeed, both salivary gland cells and motor neurons expressing $30 \mathrm{R}$ have a marked reduction in nuclear to cytoplasmic ratio of Mitf (Figure 4A-D). To assess whether this loss of nuclear Mitf leads to a decrease in CLEAR gene expression in adult heads we expressed 30R using a ubiquitous inducible driver, daughterless-GeneSwitch (daGS). In control flies, a mild ( 1.75 fold) overexpression of Mitf mRNA resulted in a significant upregulation of 3 of the 7 Mitf targets tested (the v-ATPase subunits Vha16-1, Vha68-2, and Vha44) and upregulation of 4 others (Figure 4E), although not statistically significant. Importantly, coexpression of 30R with daGS>Mitf; led to a similar $\sim 2 x$ increase in Mitf transcripts but did not induce Mitf target genes

15 (Figure 4E). This lack of Mitf target induction in 30R flies suggests that decreased nuclear import of Mitf suppresses the ability of 30R-expressing flies to upregulate CLEAR genes in order to maintain autophagic flux.

We next examined whether rescue of nucleocytoplasmic transport defects in 30Rexpressing animals can rescue Mitf nuclear localization and autophagolysosomal defects.

20 Mitf/TFEB nuclear export has recently been demonstrated to be regulated by exportin-1 (Li et al., 2018; Silvestrini et al., 2018). Knockdown of exportin-1 (Drosophila emb) rescues G4C2mediated cytoplasmic Mitf mislocalization in the salivary gland (Figure 5A-B) and GFP::Lamp accumulation in motor neurons (Figure 5C-D). Importantly, emb knockdown increases the total number of autophagosomes in G4C2-expressing motor neuron cell bodies by 3-fold (Figure 25 5E-F), suggesting that nuclear retention of Mitf rescues autophagolysosomal defects. 
bioRxiv preprint doi: https://doi.org/10.1101/2020.06.26.173021; this version posted June 26, 2020. The copyright holder for this preprint (which was not certified by peer review) is the author/funder, who has granted bioRxiv a license to display the preprint in perpetuity. It is made available under aCC-BY 4.0 International license.

Together, these data demonstrate that autophagolysosomal dysfunction in 30R-expressing animals occurs downstream of nucleocytoplasmic transport disruption.

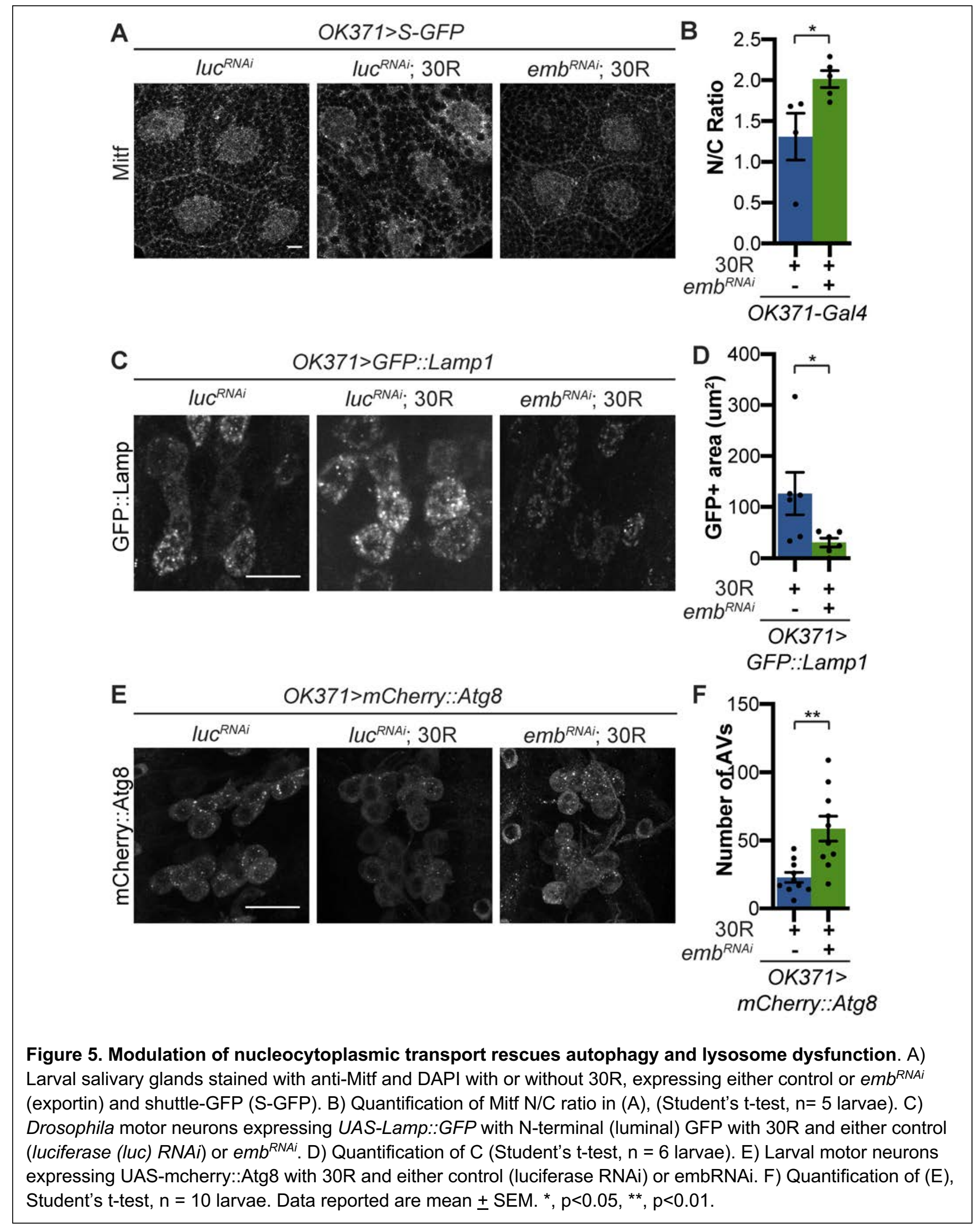




\section{Mitf rescues $\mathrm{G} 4 \mathrm{C} 2$ repeat-mediated degeneration}

Since Mitf mislocalization contributes to autophagolysosome defects in a fly C9-ALS model, we hypothesized that increasing total levels of Mitf might compensate for impaired nuclear import. While high level Mitf overexpression is toxic in Drosophila (Hallsson et al.,

5 2004), a genomic duplication of the Mitf gene (Mitf $D p$ ) is sufficient to partially rescue 30Rmediated eye degeneration, while Mitf knockdown strongly enhances eye degeneration (Figure 6A-B). Furthermore, pupal lethality caused by 30R expression in motor neurons and climbing impairment in elavGS>30R flies are also partially rescued by Mitf Dp (Figure 6C-D). Therefore, increasing Mitf levels in multiple neuronal subtypes in Drosophila suppresses G4C2-mediated neurotoxicity, consistent with our hypothesis that loss of nuclear Mitf is a key contributor to G4C2-mediated neurodegeneration.

If the impaired lysosomal function we observe in our Drosophila model is contributing to neurodegeneration downstream of NCT defects, we would predict that genetic upregulation of key regulators of lysosome function may suppress degenerative phenotypes. Indeed,

15 overexpression of rab7, the small GTPase required for fusion of autophagosomes with lysosomes, or TRPML, a lysosomal calcium channel, suppress eye degeneration (Figure S6AB). Furthermore, overexpression of key lysosomal v-ATPase subunits whose expression is regulated by Mitf also suppresses neurodegeneration in the Drosophila eye, while RNAimediated knockdown enhances degeneration ( Figure S6A-B). Interestingly, loss of the ALS-

20 associated gene ubqn in Drosophila was also rescued by increase in key lysosomal vATPases or by nanoparticle mediated lysosome acidification (Senturk et al., 2019). Overexpression of these Mitf-regulated genes also showed partial rescue of pupal lethality in animals expressing 30R in motor neurons (Figure S6C). These findings suggest a model whereby downregulation of Mitf targets leads to lysosomal disruption in G4C2 repeatexpressing flies. 


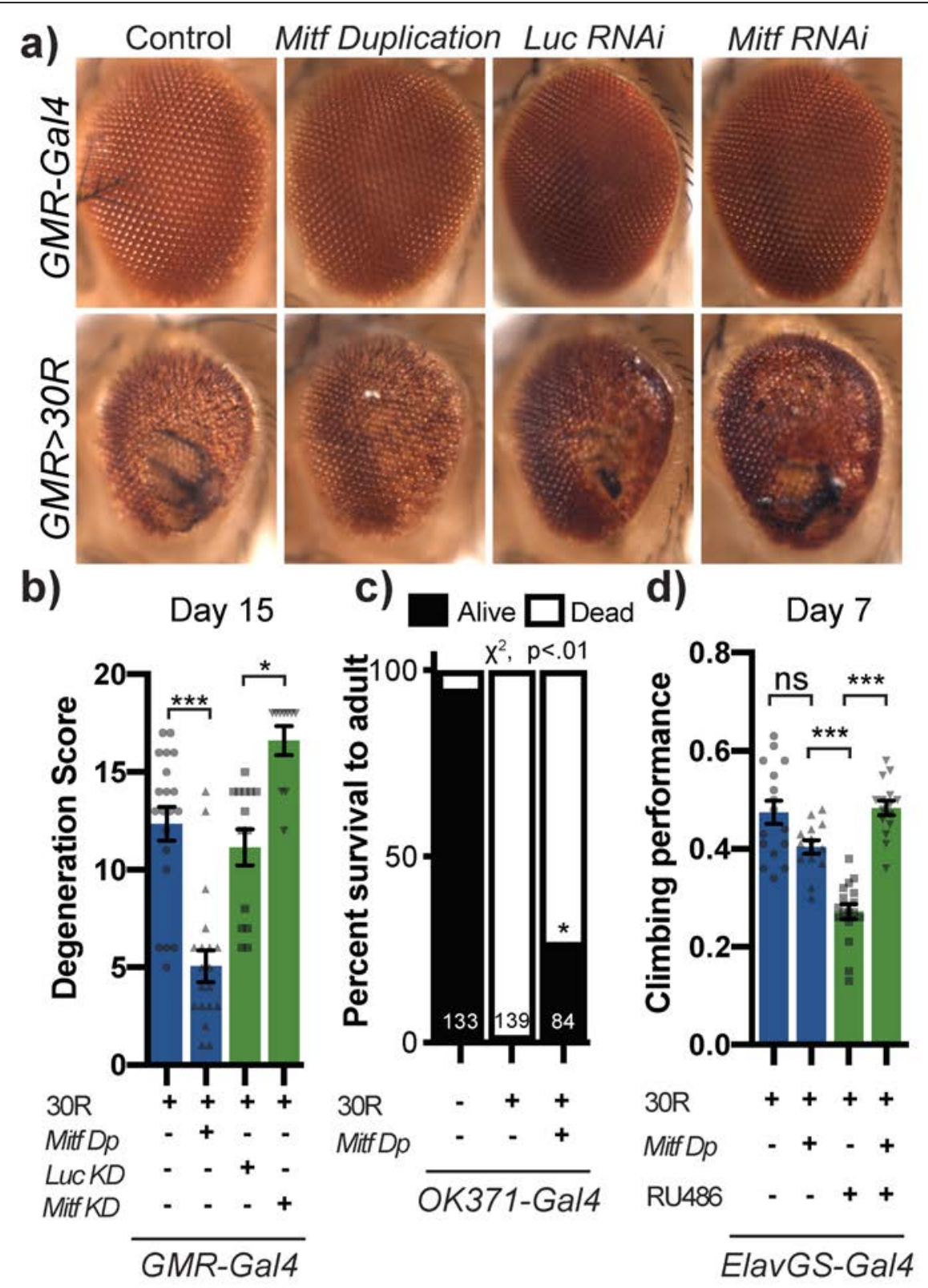

Figure 6. Transcription factor Mitf/TFEB suppresses neurodegeneration caused by G4C2 expansion. (A) 15-day old fly eyes expressing 30R under the control of GMR-Gal4, crossed to either control, genomic Mitf Duplication (Mitf Dp), Luciferase (Luc) RNAi as control, or MitfRNAi. (B) Quantification of external eye degeneration shown in (A), Kruskal-Wallis test followed by Dunn's multiple comparisons, $n=10-20$. (C) Percent of pupal eclosion in flies expressing 30R under control of OK371-Gal4 driver with Gal4 alone control, background control, or Mitf Dp, Chi-square test, $n=139,84$ respectively. (D) Adults expressing 30R under control of the inducible, pan-neuronal elavGS-Gal4 driver have decreased climbing ability at 7 days age. Co-expressing Mitf $D p$ with the 30R rather than control UAS-lacZ rescues climbing ability. Data are mean \pm SEM. n.s., not significant ${ }^{*}, p<0.05,{ }^{* * *} p<0.001$

\section{Nuclear TFEB is reduced in human cells and motor cortex with GGGGCC repeat}

\section{expansions}

In humans, TFEB is the homolog of $d$ Mitf that is best characterized for its role in 
Martini-Stoica, Xu, Ballabio, \& Zheng, 2016). Interestingly, nuclear TFEB was selectively depleted in the motor cortex of a sample of 5 ALS patients compared to 5 controls (Wang et al., 2016). To test the relevance of our findings in Drosophila models to human disease, we next examined whether G4C2 repeat expression impairs nuclear import of TFEB in HeLa cells

5 stably expressing TFEB-GFP (Roczniak-Ferguson et al., 2012) using a 47-repeat (47R) G4C2 construct that expresses tagged DPRs (See methods). TFEB-GFP is predominantly localized to the cytoplasm in both control cells and cells expressing a 47 G4C2 repeat construct (Figure 7A-B). However, induction of autophagy by 3 hours starvation leads to nuclear translocation of TFEB in control cells but not in cells expressing 47R (Figure 7A-B). We then tested the effect

10 of expression of DPRs produced by alternate codons (i.e. in the absence of G4C2 repeats): poly-(GA)50, poly-(GR)50, and poly-(PR)50 (Figure S7A-B). Only polyGA causes a mild impairment in TFEB nuclear translocation, whereas polyGR and polyPR stimulate TFEB-GFP nuclear translocation even under normal culture conditions. These data suggest that despite the protein stress response initiated by DPRs driving nuclear translocation of TFEB under

15 homeostatic conditions, human cells expressing the G4C2 repeat are unable to efficiently import TFEB into the nucleus.

To further investigate the relevance of loss of TFEB nuclear import to C9-ALS patients, we obtained human motor cortex samples from 4 non-neurological controls and 4 C9-ALS patients (Table S2). These samples were fractionated into cytoplasmic and nuclear-enriched

20 fractions and assayed for TFEB using Western analysis. TFEB is reduced by an average of $76 \%$ in the nuclear fraction and by about $50 \%$ in the cytoplasm in C9-ALS compared to controls (Figure 7C-D, Figure S7C). These data suggest that TFEB protein is downregulated in C9-ALS/FTD motor cortex, but the greatest depletion occurs in the nucleus. Therefore, we propose a model whereby disruption of protein nuclear import by the C9orf72-HRE results in a

25 failure of Mitf/TFEB to translocate to the nucleus to regulate the autophagic response to protein stress. This impairment in autophagic flux results in the accumulation of p62-positive 
bioRxiv preprint doi: https://doi.org/10.1101/2020.06.26.173021; this version posted June 26, 2020. The copyright holder for this preprint (which was not certified by peer review) is the author/funder, who has granted bioRxiv a license to display the preprint in perpetuity. It is made available under aCC-BY 4.0 International license.

ubiquitinated aggregates that contribute to a feed-forward cycle of proteostasis disruption and

chronic cell stress signaling, eventually leading to neuron cell death.

A

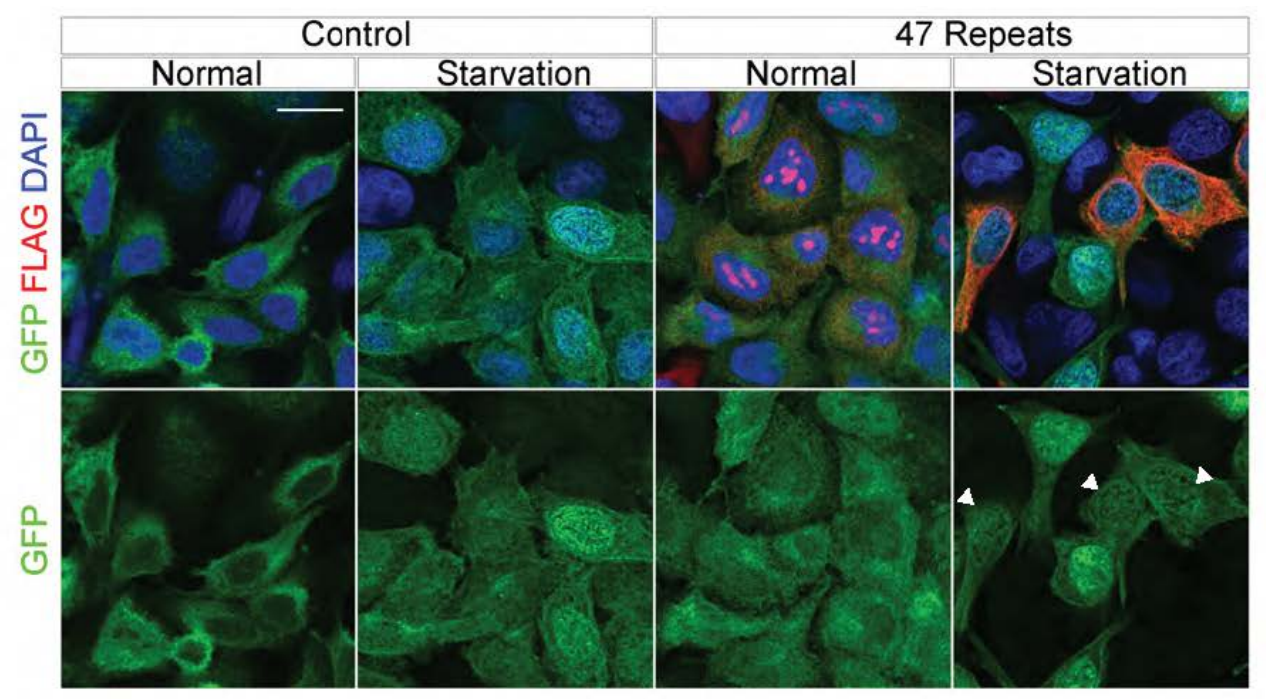

B
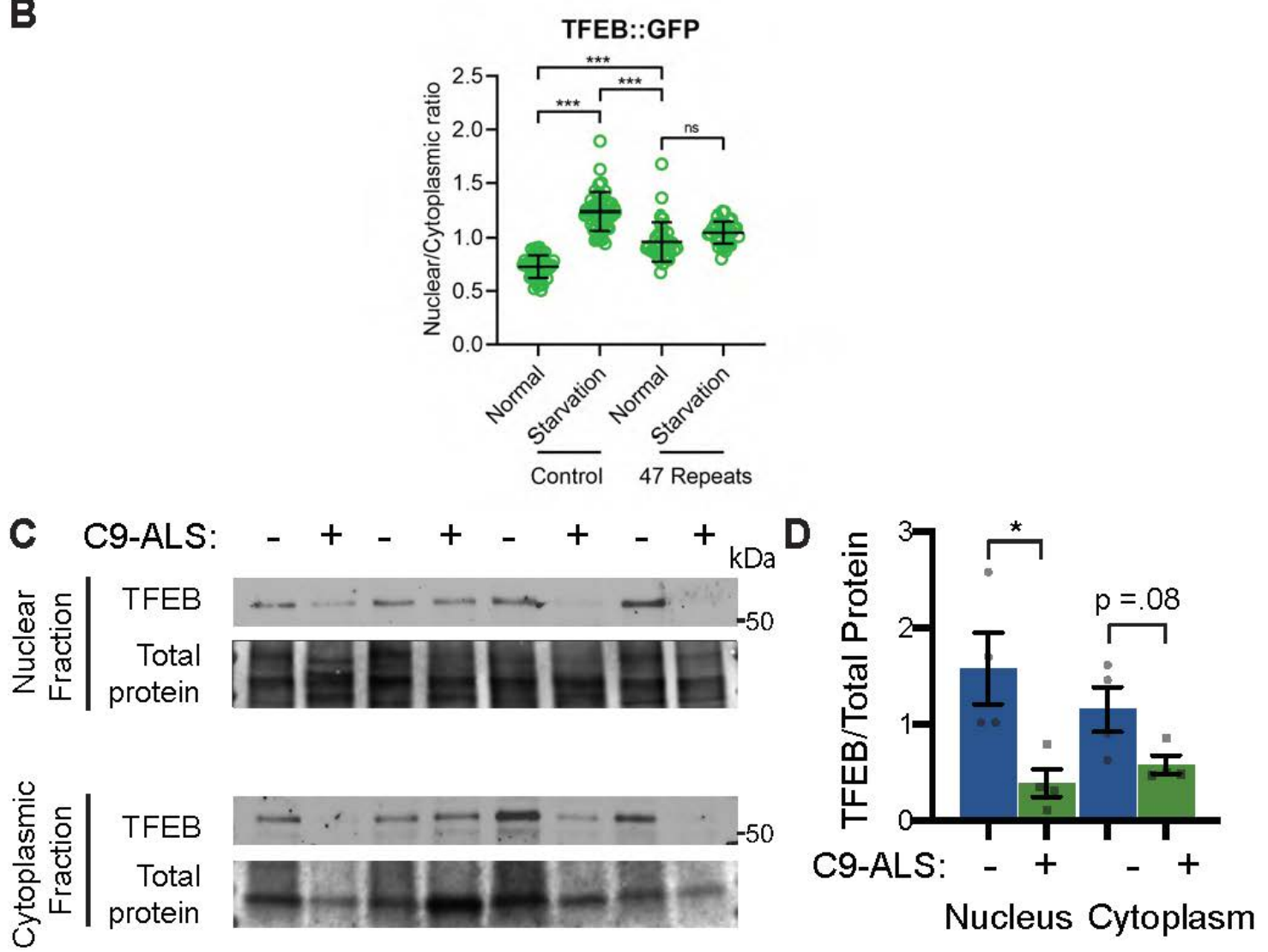

Figure 7. Nuclear TFEB is reduced in human cells expressing GGGGCC and C9-ALS human motor cortex. (A) HeLa cells stably expressing TFEB::GFP transfected with OR (Control) or a 47R construct in normal (DMEM) or starved (3 hour EBSS) conditions. White arrows indicate transfected cells in the 47R starved group. (B) Quantification of cells from (A) showing the nucleocytoplasmic ratio of TFEB for each group $(n=n=47,47,35,38$ cells, Kruskal-Wallis test with Dunn's multiple comparisons). (C) Western blot for TFEB of human motor cortex samples fractionated into cytoplasmic and nuclear samples from non-neurological controls and C9-ALS patients. (B) Quantification of TFEB levels against total protein loading (Faststain) in non-neurological controls and C9-ALS patients $\left(N=4\right.$, Student's t-test). Data reported are mean \pm SEM. n.s., not significant ${ }^{*}, p<0.05,{ }^{* * *} p<0.001$. 


\section{Discussion}

Our work has revealed that the ALS-associated G4C2 hexanucleotide repeat is sufficient to disrupt multiple aspects of autophagy. In Drosophila, G4C2 repeats cause loss of autophagosomes and disrupt lysosomal structure and function. This accumulation of

5 autolysosomes and lysosome-related organelles called multilamellar bodies (MLBs) has been observed in lysosomal storage disorders and has been reported in spinal cord tissue from sporadic ALS patients (Bharadwaj, Cunningham, Zhang, \& Lloyd, 2016; Parkinson-Lawrence et al., 2010; Sasaki, 2011). Regulation of protein and lipid homeostasis by the lysosome may be particularly important in neurons since they are post-mitotic and have high energy demands

10 (Fraldi, Klein, Medina, \& Settembre, 2016). Loss of function of C9ORF72 also disrupts autophagy and lysosomal function in multiple cell types (Farg et al., 2014; Ji, Ugolino, Brady, Hamacher-Brady, \& Wang, 2017; O'Rourke et al., 2015; Sellier et al., 2016; Y. Shi et al., 2018; Sullivan et al., 2016; Ugolino et al., 2016; Webster et al., 2016; Yang et al., 2016; Zhu et al., 2020), suggesting a mechanism whereby G4C2 repeats may have synergistically detrimental

15 effects with haploinsufficient C9ORF72 in C9-ALS/FTD patients. Additionally, multiple forms of familial ALS are caused by genes that regulate autophagy and lysosome function (Evans \& Holzbaur, 2019; Lin et al., 2017; Ramesh \& Pandey, 2017), and upregulation of lysosome function has been proposed to be beneficial in multiple preclinical models of ALS (Donde et al., 2020; Mao et al., 2019; Senturk et al., 2019; Y. Shi et al., 2018). Thus, our findings suggest

20 that like other forms of ALS, neurotoxicity of G4C2 repeats in C9 ALS-FTD is at least partially caused by disrupted autophagolysosomal function.

The finding that ref(2)p knockdown prevents or delays G4C2-mediated neurodegeneration is surprising, as p62/SQSTM1 is thought to link toxic ubiquitinated aggregates to LC3 to remove aggregates via selective autophagy (Cipolat Mis, Brajkovic,

25 Frattini, Di Fonzo, \& Corti, 2016; Levine \& Kroemer, 2008; Saitoh et al., 2015). 
bioRxiv preprint doi: https://doi.org/10.1101/2020.06.26.173021; this version posted June 26, 2020. The copyright holder for this preprint (which was not certified by peer review) is the author/funder, who has granted bioRxiv a license to display the preprint in perpetuity. It is made available under aCC-BY 4.0 International license.

However, other studies have also suggested that p62 may contribute to (rather than

ameliorate) toxicity of ubiquitinated protein aggregates. For example, $\operatorname{Atg} 7^{-/-}$mice display

severe defects in autophagy and accumulation of p62-positive protein aggregates in the liver and brain, and knockout of p62 in these mice prevents the formation of ubiquitinated

5 aggregates and rescues liver dysfunction via suppression of chronic oxidative stress signaling (Komatsu et al., 2007). Additionally, ATM-mediated DNA double stranded break repair is impaired in cultured neurons expressing the C9orf72-HRE, and this phenotype is rescued by p62 knockdown (Walker et al., 2017). These findings suggest that accumulation of p62-positive aggregates may contribute to DNA damage previously described in C9-ALS. Further, p62 is

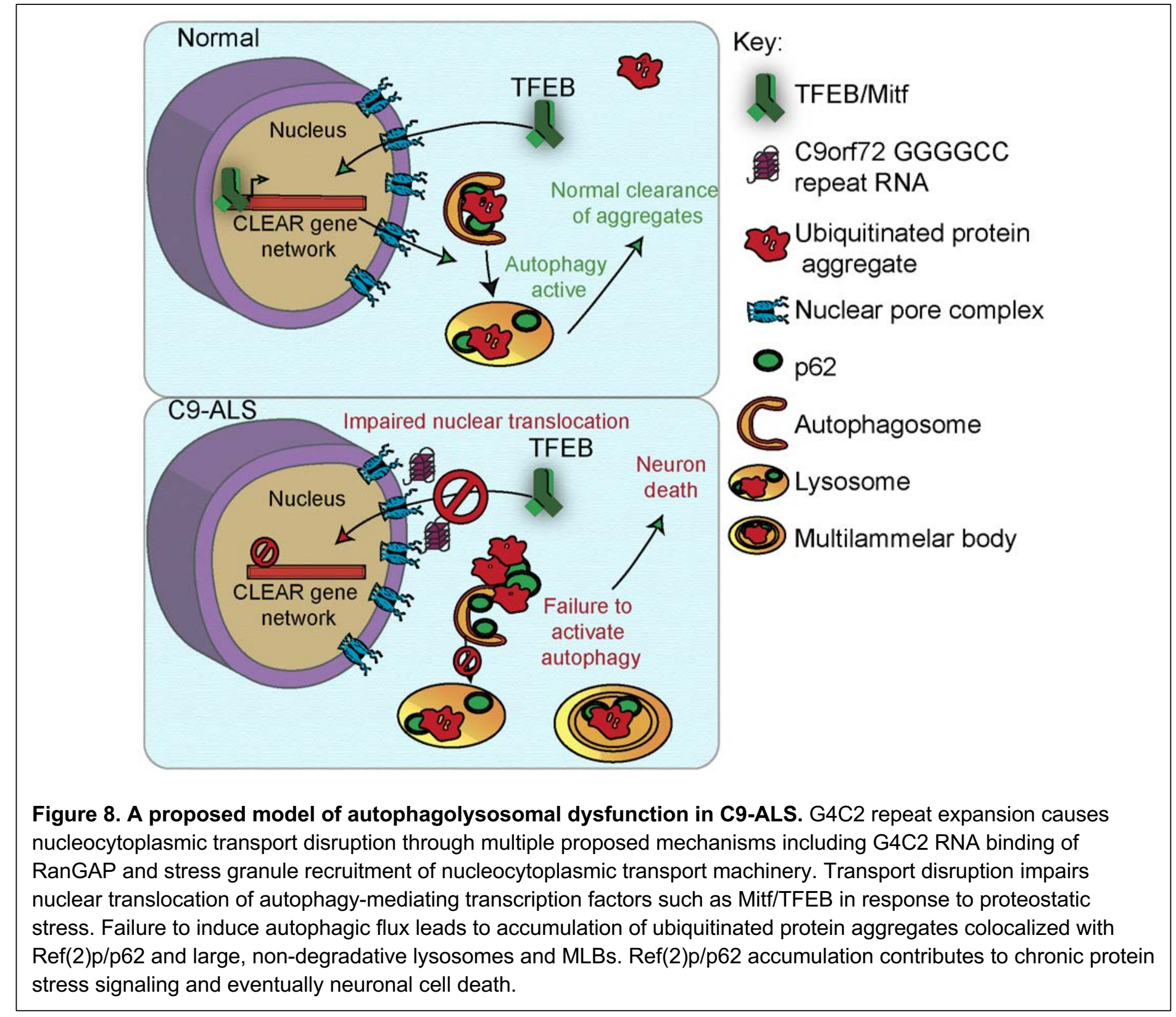


found to co-localize with DPRs in C9-ALS patients (Al-Sarraj et al., 2011; Mackenzie et al.,

2014; Mori et al., 2013) and may promote protein aggregation. We hypothesize that p62-

positive aggregate formation in C9-patients contributes to neurotoxicity by activating

downstream signaling pathways that are alleviated by autophagy-mediated aggregate

5 clearance.

While many groups have reported nucleocytoplasmic transport dysfunction in ALS, it has remained unclear how NCT disruption causes ALS. Stress granules can recruit nuclear pore proteins to the cytoplasm and cause nucleocytoplasmic transport defects, suggesting that the disruptions in phase separation of RNA binding proteins may lie upstream of that hyperactivity of nonsense-mediated decay (NMD) may lie downstream of nucleocytoplasmic transport, indicating that multiple proteostasis pathways may be disrupted (Ortega et al., 2020). Additionally, selective autophagy has been proposed to be required for nuclear pore turnover (Lee et al., 2020), implying that autophagy defects may contribute to the

15 cytoplasmic nuclear pore pathology found in C9-ALS patients and animal models. Our data show that in Drosophila, HeLa cells, and C9-ALS postmortem brain tissue, nucleocytoplasmic transport defects lead to an inability to activate TFEB translocation to the nucleus, causing widespread autophagy defects and accumulation of protein aggregates (Figure 8). These findings place nucleocytoplasmic transport defects in ALS upstream of proteostasis defects. Importantly, TFEB has been previously proposed as a therapeutic target in ALS and other neurodegenerative disease (Cortes \& La Spada, 2019). Upregulation of TFEB signaling helps clear multiple types of proteotoxic aggregates found in Alzheimer's disease, Parkinson's disease, Huntington's disease, ALS and FTD (Decressac et al., 2013; Parr et al., 2012; Polito et al., 2014; Torra et al., 2018; Vodicka et al., 2016). Our study suggests that modulation of both nucleocytoplasmic transport and autophagy may act synergistically in ALS and FTD. 


\section{Materials and Methods}

\section{Drosophila genetics}

5 A complete list of fly lines used in this study is described in Supplemental Table 3. Drosophila were raised on standard cornmeal-molasses food at $25^{\circ} \mathrm{C}$. For eye degeneration, GMR-GAL4, UAS-30R/CyO, twi-GAL4, UAS-GFP were crossed to UAS-modifier lines or background controls and GMR-GAL4, UAS-30R/UAS-modifier or GMR-GAL4, UAS-30R/+ were selected (where UAS-modifier can be on any chromosome) from the offspring and aged at $25^{\circ} \mathrm{C}$ for 15 days. Eye degeneration is quantified using a previously described method ${ }^{89}$. Briefly, points were added if there was complete loss of interommatidial bristles, necrotic patches, retinal collapse, loss of ommatidial structure, and/or depigmentation of the eye. Eye images were obtained using a Nikon SMZ 1500 Microscope and Infinity 3 Luminera Camera with Image Pro Insight 9.1 software.

For pupal survival assay, either 3 males from OK371-Gal4 or OK371-Gal4; UAS30R/TM6G80(Tb) were crossed to 5-6 female flies containing UAS-modifier lines or background controls. Parental adult crosses were transferred to fresh vials every 2-3 days. After 15 days, non-tubby pupated flies that were (either OK371-Ga/4/UAS-modifier, OK371/+; UAS-30R, or OK371-Gal4/UAS-modifier; UAS-30R were scored as either eclosed (empty pupal case) or non-eclosed (typically a fully developed pharate adult fly unable to eclose from pupal case due to paralysis).

For the climbing assay, UAS-30R; elavGS-Gal4 were crossed to experimental or genetic background controls. Adults were transferred 3-5 days after eclosion to vials containing 200 M RU486 food or ethanol vehicle alone and transferred to new vials every 2-3 days. After

25 aging 7-10 days, groups of 10 flies were placed into empty food vials and were tapped to the bottom and then locomotor function assessed by their negative geotaxis (flies reflexively crawl 
against gravity) response as measured by ability to climb $8 \mathrm{~cm}$ in 10 seconds. Each cohort of 10 flies was tested 10 times to obtain an average. $\mathrm{N}$ represents individual cohorts of 10 flies.

\section{Drosophila drug feeding}

5 Cornmeal-molasses-yeast fly food was melted and then cooled for 5 minutes before being mixed with concentrations of mifepristone (RU486, Sigma), rapamycin (Selleckchem), or trehalose (Sigma) and cooled to room temperature. Ethanol or DMSO was used as a vehicle control. Parent flies were crossed on normal food, and then they were transferred to food containing drug every 2-3 days such that their offspring would develop in food containing drug or adult offspring were transferred to drug food once eclosed as noted. Wandering third instars were selected for immunostaining or western blot analysis. Adult flies were aged on the drug-containing food for 15 days before analyzing their eye morphology or assessed for climbing ability on the day noted.

\section{Quantitative RT-PCR}

For each genotype, mRNA was collected from 5 flies using the TRIzol reagent (Thermo Fischer Scientific) following the manufacturer's protocol. Reverse transcription was performed using SuperScript III First-Strand synthesis kit (Thermo Fischer Scientific) following the manufacturer's protocol. Quantitative PCR was performed using SYBR Green PCR system

20 (Applied Biosystem) on a 7900HT fast Real-Time PCR system (Applied Biosystem). Primers are listed in Supplemental Table 4. The primers for G4C2 repeats were designed to amplify a 3' region immediately after the repeats in the UAS construct.

\section{Immunofluorescence staining and imaging}

25 For Drosophila ventral nerve cords, wandering $3^{\text {rd }}$ instar larvae were dissected in $\mathrm{HL}^{90}$ using a standard larval fillet dissection then fixed in $4 \%$ paraformaldehyde (or, for UAS-Atg8-Cherry 
experiments Bouin's fixative (Sigma)) for 20 minutes, followed by wash and penetration with

PBS $0.1 \%$ Triton X-100 (PBX) for 3x 20-minute washes. The tissues were blocked for 1 hour at room temperature in PBS with 5\% normal goat serum (NGS) and $0.1 \%$ PBX, then stained with the following primary antibodies at 4C overnight (16h): rabbit anti-dsRed (Clontech) 1:1,000;

5 mouse anti-poly ubiquitin FK1 (Enzo Life Sciences) 1:200; rabbit anti-Ref2p (a gift from Gabor Juhasz, Eotvos Lorand University, Budapest, Hungary]; guinea pig anti-Mitf [a gift from Francesca Pignoni, SUNY Upstate, New York, USA) 1:500; rat anti-HA (Sigma) 1:200. Tissues were washed 3 times for 20 minutes each with 0.1\% PBX. Secondary antibodies (Goat antibodies conjugated to Alexa Fluor 568, 488, 633) diluted in 0.1\% PBX 5\% NGS and

10 incubated for $2 \mathrm{~h}$ and then washed 3 times for 20 minutes each with $0.1 \%$ PBX. During one wash, DAPI was added to the prep at a final concentration of $1 \mathrm{ug} / \mathrm{mL}$. Larvae were mounted in Fluoromount-G (Invitrogen).

Drosophila salivary glands were dissected using a standard protocol and stained as above excepting for stronger solubilization with $0.3 \%$ PBX. Fixed cells or tissues were

15 analyzed under an LSM780 or LSM800 confocal microscope (Carl Zeiss) with their accompanying software using Plan Apochromat $63 \times$, NA 1.4 DIC or Plan Apochromat 40×, 1.3 Oil DIC objectives (Carl Zeiss) at room temperature. Images were captured by an AxioCam HRc camera (Carl Zeiss) and were processed using ImageJ/Fiji (National Institutes of Health). To quantify fluorescent intensities, after opening the images in ImageJ/Fiji, certain areas/bands

20 were circled and the intensities were measured. Puncta were counted using the Analyze Particles function in Image $\mathrm{J}$ using the same thresholding across experiments. Images are representative and experiments were repeated two to five times.

\section{Western Blotting}

25 Tissues or cells were homogenized and/or lysed in RIPA buffer (50 mM Tris- $\mathrm{HCl}$ pH 7.4, $150 \mathrm{mM} \mathrm{NaCl}, 0.1 \%$ SDS, $0.5 \%$ sodium deoxycholate, and $1 \%$ Triton $\mathrm{X}-100$ ) supplemented 
with protease inhibitor cocktail (Complete, Roche) using microcentrifuge pestles, and then were incubated in RIPA buffer on ice for 20 minutes. Samples were spun down at $100 \mathrm{~g}$ for 5 minutes to remove carcass and unbroken cells. For protein quantification, solution was diluted and measured by BCA assay (Thermo Fischer Scientific).

For nucleocytoplasmic fractionation of autopsy tissue, fractionation was performed with the NE-PER Nuclear and Cytoplasmic Extraction Kit \#78833 (Thermo Fischer Scientific) according to the manufacturer's protocol. For detection of proteins in the whole fraction, Drosophila larvae were solubilized in 8M urea. For the soluble and pelleted fraction, larvae were first solubilized in RIPA buffer as described above. The samples were spun down at $15000 \mathrm{rpm}$ for 20 minutes and the soluble supernatant was set aside. Freshly prepared 8M urea buffer (Sigma) was added to the pellet and dissolved through vortexing. Samples were spun again at 15000rpm for 20 minutes and urea-soluble pellet fraction was collected. A small amount of sample buffer dye was added and urea-buffered protein samples were run immediately on SDS-PAGE without heating. For immunoblot, 10-50ug of

15 total protein sample was mixed with 4x Laemmli buffer (Bio-Rad) and heated at $98{ }^{\circ} \mathrm{C}$ for 10 min. The protein samples were run on 4-15\% SDS Mini-PROTEAN TGX Precast Gels (BioRad) and transferred to nitrocellulose membrane. TBST (50 mM Tris- $\mathrm{HCl} \mathrm{pH} 7.4,1 \%$ Triton X-100) with 5\% non-fat milk (Bio-Rad) was used for blocking. Primary antibodies were used as below: rabbit anti-Ref(2)p [a gift from Gabor Juhasz, Eotvos Lorand University, Budapest, 20 Hungary] 1:1000; chicken anti-GFP (Abcam) 1:1000; guinea pig anti-CP1 [a gift from Patrick Dolph, Dartmouth College, NH, USA]; mouse anti-poly ubiquitin FK1 (Enzo Life Sciences) 1:1000; mouse anti- beta actin (clone C4) (EMD Millipore); rabbit anti-TFEB (Bethyl Laboratories) 1:2000, rabbit anti- histone H3 (Abcam) 1:1000.

\section{Electroretinogram (ERG) Assay}


For ERG recordings, Rh1-GAL4/UAS-LacZ and Rh1-GAL4/UAS-(G4C2) 30 flies were aged at

$25^{\circ} \mathrm{C}$ in $12 \mathrm{~h}$ light/12h dark cycle. ERG recordings were performed as described ${ }^{66}$. In brief, adult flies were immobilized on a glass slide by glue. A reference electrode was inserted in the thorax and a recording electrode was placed on the eye surface. Flies were maintained in the

5 darkness for at least 2 minutes prior to 1-second flashes of white light pulses (LED source with daylight filter), during which retinal responses were recorded and analyzed using WinWCP (University of Strathclyde, Glasgow, Scotland) software. At least 5 flies were examined for each genotype and timepoint.

\section{Transmission Electron Microscopy (TEM)}

10 rh1-GAL4/UAS-lacZ and $r h 1-G A L 4 / U A S-(G 4 C 2)_{30}$ flies were aged at $25^{\circ} \mathrm{C}$ in $12 \mathrm{~h}$ light $/ 12 \mathrm{~h}$ dark cycle. Retinae of adult flies were processed for TEM imaging as previously described (Chouhan et al., 2016). 3 flies were examined for each genotype and timepoint.

\section{Plasmids Source and Construction}

15 pSF-CAG-Amp (0G504) were purchased from Oxford Genetics. We generated a mammalian expressing plasmid pSF-(G4C2)47-VFH (V5-Flag-His), which can express 47 HRE repeats with 3 different tags to monitor expression of DPRs (polyGA, polyGP, and polyGR). pEGFP. (GA, GR, or PR)50 was obtained from Davide Trotti (Wen et. al 2014) and the GFP cDNA sequence was replaced with mCherry by digesting with BamHI and Xhol.

\section{TFEB-GFP Hela cell culture and transfection}

Hela cell line with stable expressing TFEB::GFP was a gift from Dr. Shawn Ferguson at Yale University. Hela cells were grown in DMEM media (Invitrogen) supplemented with 10\% fetal bovine serum (Hyclone Laboratories Inc.) as previous described ${ }^{93}$. Transfection was performed 
$\mu \mathrm{g}$ of cDNA was diluted into $100 \mu \mathrm{l}$ of Opti-MEM I Medium (Invitrogen) and mixed gently.

Lipofectamine 2000 mixture was prepared by diluting 2-4 $\mu$ l of Lipofectamine 2000 in $100 \mu l$ of Opti-MEM I Medium. The ratio of DNA to Lipofectamine 2000 used for transfection was 1:2 as indicated in the manual. The DNA-Lipofectamine 2000 mixture was mixed gently and

5 incubated for $20 \mathrm{~min}$ at room temperature. Cells were directly added to the $200 \mu$ l of DNALipofectamine 2000 mixture. After 48 h, transfected Hela cells were treated with EBSS medium for $3 \mathrm{~h}$ for starvation.

\section{Immunofluorescence analysis of Hela cells}

10 Hela cells were fixed with 4\% PFA at room temperature for 15 min, washed three times with PBS, permeabilized for 10 min with 1\% PBTX, washed another three times with PBS, and blocked for $1 \mathrm{hr}$ at room temperature with $10 \%$ normal goat serum (Sigma) diluted in $0.1 \%$ PBTX. Cells were then incubated overnight at $4^{\circ} \mathrm{C}$ with primary antibody mouse anti-Flag antibody (1:1000, Sigma). After three washes in PBS (5 min each), cells were incubated for 1

$15 \mathrm{hr}$ at room temperature with secondary antibodies (goat anti-Alexa Fluor 568, 1:250; Invitrogen) diluted in the blocking solution. Cells were washed three times in PBS and mounted with Prolong Gold anti-fade reagent with DAPI (Cell Signaling).

\section{Antibodies}

20 For immunofluorescence experiments, the following antibodies were used: Rabbit anti-dsRed (Clontech \#632496) 1:1,000; mouse anti-poly ubiquitin FK1 (Enzo Life Sciences \#BMLPW8805) 1:200; rabbit anti-Ref2p ${ }^{93}$ (a gift from Gabor Juhasz, Eotvos Lorand University, Budapest, Hungary) 1:1000; guinea pig anti-Mitf ${ }^{39}$ (a gift from Francesca Pignoni, SUNY Upstate, New York, USA) 1:500; rat anti-HA (Sigma \#11867423001) 1:200; mouse anti-Flag

25 antibody (Sigma \#F3165) 1:1000. For Western experiments: rabbit anti-Ref(2)p (from Gabor Juhasz), 1:1000; chicken anti-GFP (Abcam ab13970) 1:1000; guinea pig anti-CP156 (a gift 
from Patrick Dolph, Dartmouth College, NH, USA); mouse anti-poly ubiquitin FK1 (Enzo Life

Sciences \#BML-PW8805) 1:1000; mouse anti- beta actin (clone C4) (EMD Millipore,

MAB1501); rabbit anti-TFEB (Bethyl A303-673A) 1:2000; rabbit anti-histone H3 (Abcam)

1:1000.

\section{Collection of human autopsied tissue.}

Human autopsied tissue used for these data are described in detail in Supplementary Table 2.

The use of human tissue and associated decedents' demographic information was approved by the Johns Hopkins University Institutional Review Board and ethics committee (HIPAA

Form 5 exemption, Application 11-02-10-01RD) and from the Ravitz Laboratory (UCSD) through the Target ALS Consortium.

\section{Statistics}

All quantitative data were derived from independent experiments. Each $\mathrm{n}$ value representing

15 biological replicates is indicated in the figure legends. Statistical tests were performed in Prism version 8.3.1 or Microsoft Excel 16.34 and were performed as marked in the figure legends. All statistical tests were two-sided. Results were deemed significant when the P value $\alpha=0.05$, $(p)^{*}=p<0.05,{ }^{* *}=p<0.01,{ }^{* * *}=p<0.001$, n.s. $=$ not significant. No statistical methods were used to predetermine sample size. The investigators were not blinded during experiments.

\section{Reagent Table}

\begin{tabular}{|c|c|c|}
\hline REAGENT or RESOURCE & SOURCE & IDENTIFIER \\
\hline \multicolumn{3}{|l|}{ Antibodies } \\
\hline Rabbit polyclonal anti-dsRed & Clontech & $\begin{array}{l}\text { Cat\#632496; } \\
\text { RRID:AB 10013483 }\end{array}$ \\
\hline Mouse monoclonal anti- poly-ubiquitin & Enzo Life Sciences & $\begin{array}{l}\text { Cat\# BML-PW8805, } \\
\text { RRID:AB_10541434 }\end{array}$ \\
\hline Rabbit polyclonal anti-Ref(2)p & Gabor Juhasz & $\mathrm{N} / \mathrm{A}$ \\
\hline Guinea pig anti-Mitf & $\begin{array}{l}\text { Francesca Pignoni (Zhang et al. } \\
2015 b)\end{array}$ & $\mathrm{N} / \mathrm{A}$ \\
\hline
\end{tabular}


bioRxiv preprint doi: https://doi.org/10.1101/2020.06.26.173021; this version posted June 26, 2020. The copyright holder for this preprint (which was not certified by peer review) is the author/funder, who has granted bioRxiv a license to display the preprint in perpetuity. It is made available under aCC-BY 4.0 International license.

\begin{tabular}{|c|c|c|}
\hline Rat anti-HA & Roche & $\begin{array}{l}\text { Cat\# 11867423001, } \\
\text { RRID:AB_390918 }\end{array}$ \\
\hline Chicken anti-GFP & Abcam & $\begin{array}{l}\text { Cat\# ab13970, } \\
\text { RRID:AB_300798 }\end{array}$ \\
\hline Guinea pig anti-CP1 & Patrick Dolph & $\mathrm{N} / \mathrm{A}$ \\
\hline Mouse anti- beta actin (clone C4) & EMD Millipore & $\begin{array}{l}\text { Cat\# MAB1501, } \\
\text { RRID:AB_2223041 }\end{array}$ \\
\hline Rabbit polyclonal anti- TFEB & Bethyl Biosciences & $\begin{array}{l}\text { Cat\# A303-673A, } \\
\text { RRID:AB_11204751 }\end{array}$ \\
\hline Rabiit polyclonal anti-Histone $\mathrm{H} 3$ & Cell Signaling & $\begin{array}{l}\text { Cat\# 9715, } \\
\text { RRID:AB_331563 }\end{array}$ \\
\hline Mouse monoclonal anti-FLAG & Sigma Aldrich & $\begin{array}{l}\text { Cat\# F3165, } \\
\text { RRID:AB_259529 }\end{array}$ \\
\hline \multicolumn{3}{|l|}{ Bacterial and Virus Strains } \\
\hline $\begin{array}{l}\text { One Shot }{ }^{\mathrm{TM}} \text { TOP10 Chemically Competent } E \text {. } \\
\text { coli }\end{array}$ & Thermo Fischer Scientific & Cat\# C404006 \\
\hline \multicolumn{3}{|l|}{ Biological Samples } \\
\hline $\begin{array}{l}\text { Control (non-neurological) and ALS Motor } \\
\text { Cortex Tissue }\end{array}$ & $\begin{array}{l}\text { Ravitz laboratory (UCSD) } \\
\text { through Target ALS Consortium; } \\
\text { Brain Resource Center at JHMI }\end{array}$ & $\mathrm{N} / \mathrm{A}$ \\
\hline \multicolumn{3}{|c|}{ Chemicals, Peptides, and Recombinant Proteins } \\
\hline Mifepristone (RU486) & Millipore Sigma & Cat \#M8046 \\
\hline Rapamycin & Selleckchem & Cat \#S1039 \\
\hline $\mathrm{D}-(+)$-Trehalose dihydrate & Millipore Sigma & Cat \#T0167 \\
\hline TRIzol & Thermo Fischer Scientific & Cat \# 15596018 \\
\hline Faststain & G-Biosciences & Cat \#786-34 \\
\hline SYBR Select Master Mix & Thermo Fischer Scientific & Cat \#4472908 \\
\hline Protease Inhibitor Cocktail & Roche & Cat\#11873580001 \\
\hline \multicolumn{3}{|l|}{ Critical Commercial Assays } \\
\hline \multirow{2}{*}{$\begin{array}{l}\text { SuperScript }{ }^{\mathrm{TM}} \text { III First-Strand Synthesis } \\
\text { System } \\
\text { NE-PER Nuclear and Cytoplasmic Extraction } \\
\text { Kit }\end{array}$} & Thermo Fischer Scientific & Cat \#18080051 \\
\hline & Thermo Fischer Scientific & Cat \#78833 \\
\hline BCA Assay & Thermo Fischer Scientific & Cat \# 23227 \\
\hline 4-15\% Mini-PROTEAN TGX Precast Gel & Bio-Rad & Cat \#4561083 \\
\hline Blotting Grade Blocker (nonfat dry milk) & Bio-Rad & Cat \#1706404 \\
\hline Lipofectamine 2000 & Thermo Fischer Scientific & Cat \#11668019 \\
\hline \multicolumn{3}{|l|}{ Experimental Models: Cell Lines } \\
\hline Human: HeLa stably expressing TFEB::GFP & $\begin{array}{l}\text { Shawn Ferguson (Roczniak- } \\
\text { Ferguson et al. 2012) }\end{array}$ & $\mathrm{N} / \mathrm{A}$ \\
\hline \multicolumn{3}{|c|}{ Experimental Models: Organisms/Strains (see also Table S3) } \\
\hline GMR-Gal4 & Bloomington Drosophila Stock Center & $\begin{array}{l}\text { BDSC:1104; } \\
\text { FlyBase: } \\
\text { FBti0002994 }\end{array}$ \\
\hline 30R: w; UAS- $\left(G_{4} C_{2}\right)_{30}$ & Jeng Jin (Xu et al. 2013) & $\begin{array}{l}\text { FlyBase: } \\
\text { FBal0294759 }\end{array}$ \\
\hline TRiP background control & Bloomington Drosophila Stock Center & BDSC: 36303 \\
\hline UAS-Ref(2)p $p^{R N A i} 1$ & Bloomington Drosophila Stock Center & BDSC: 36111 \\
\hline UAS-Ref(2)p-HA & -M. Martins (de Castro et al. 2013) & $\begin{array}{l}\text { Flybase: } \\
\text { FBtp0089618 }\end{array}$ \\
\hline vGlut-OK371-Gal4 & Bloomington Drosophila Stock Center & $\begin{array}{l}\text { Flybase: } \\
\text { FBal0194519 }\end{array}$ \\
\hline$U A S-R e f(2) p^{R N A i} 2$ & Bloomington Drosophila Stock Center & BDSC: 33978 \\
\hline
\end{tabular}


bioRxiv preprint doi: https://doi.org/10.1101/2020.06.26.173021; this version posted June 26, 2020. The copyright holder for this preprint (which was not certified by peer review) is the author/funder, who has granted bioRxiv a license to display the preprint in perpetuity. It is made available under aCC-BY 4.0 International license.

\begin{tabular}{|c|c|c|}
\hline elavGS-Gal4 & $\begin{array}{l}\text { Adrian Isaacs laboratory } \\
\text { (Mizielinska et al. 2014) }\end{array}$ & $\begin{array}{l}\text { Flybase: } \\
\text { FBtp0015149 }\end{array}$ \\
\hline $\operatorname{Ref}(2) p^{\text {od3 }}$ & Eric Baehrecke (Shravage et al. 2013) & $\begin{array}{l}\text { Flybase: } \\
\text { FBal0032568 }\end{array}$ \\
\hline$U A S-G R_{36}$ & Adrian Isaacs (Mizielinska et al 2014) & $\mathrm{N} / \mathrm{A}$ \\
\hline Act-Gal4 & Bloomington Drosophila Stock Center & $\begin{array}{l}\text { Flybase: } \\
\text { FBti0183703 } \\
\end{array}$ \\
\hline UAS-Ref(2)p-GFP & & $\begin{array}{l}\text { Flybase: } \\
\text { FBtp0041098 }\end{array}$ \\
\hline UAS-mCherry-Atg8 & Bloomington Drosophila Stock Center & BDSC: 37749 \\
\hline UAS-GFP-Lamp & $\begin{array}{l}\text { Helmut Kramer (Pulipparacharuvil } \\
\text { et al. 2005) }\end{array}$ & $\begin{array}{l}\text { Flybase: } \\
\text { FBtp0041063 }\end{array}$ \\
\hline UAS-3R; UAS-( $\left(\mathrm{G}_{4} \mathrm{C}_{2}\right)_{3}$ & Adrian Isaacs (Mizielinska et al. 2014) & $\begin{array}{l}\text { Flybase: } \\
\text { FBal0304407 }\end{array}$ \\
\hline UAS-36R; UAS- $\left(\mathrm{G}_{4} \mathrm{C}_{2}\right)_{36}$ & Adrian Isaacs (Mizielinska et al. 2014) & $\begin{array}{l}\text { Flybase: } \\
\text { FBti0166223 }\end{array}$ \\
\hline UAS-lacZ & Bloomington Drosophila Stock Center & BDSC: 3956 \\
\hline rh1-Gal4 & Bloomington Drosophila Stock Center & BDSC: 8961 \\
\hline gRab7-YFP & Bloomington Drosophila Stock Center & BDSC: 62545 \\
\hline UAS-Rab7-GFP & Bloomington Drosophila Stock Center & BDSC: 42706 \\
\hline UAS-luciferase ${ }^{R N A i}$ & Bloomington Drosophila Stock Center & BDSC: 31603 \\
\hline UAS-S-GFP (UAS-NLS,NES-GFP) & Bloomington Drosophila Stock Center & BDSC: 7032 \\
\hline UAS-RanGAP & Lloyd laboratory (Zhang et. al 2015) & $\mathrm{N} / \mathrm{A}$ \\
\hline UAS-RanGAPRNAi & Bloomington Drosophila Stock Center & BDSC: 29565 \\
\hline UAS-CD8-GFP & Bloomington Drosophila Stock Center & $\begin{array}{l}\text { Flybase: } \\
\text { FBti0012685 }\end{array}$ \\
\hline UAS-Mitf-HA & Francesca Pignoni (Zhang et al. 2015b) & $\mathrm{N} / \mathrm{A}$ \\
\hline daGS-Gal4 & & $\begin{array}{l}\text { Flybase: } \\
\text { FBtp0057039 }\end{array}$ \\
\hline UAS-embargoed ${ }^{R N A i}$ & Bloomington Drosophila Stock Center & BDSC: 31353 \\
\hline Mitf duplication; Mitf ${ }^{S-R E S}$ & Francesca Pignoni (Zhang et al. 2015b) & $\begin{array}{l}\text { Flybase: } \\
\text { FBtp0115483 }\end{array}$ \\
\hline UAS-Mitf ${ }^{R N A i}$ & Bloomington Drosophila Stock Center & BDSC: 43998 \\
\hline UAS-Mitf RNAi 2 & Vienna Drosophila Resource Center & VDRC: 108519 \\
\hline UAS-Mitf ${ }^{R N A i} 3$ & Bloomington Drosophila Stock Center & BDSC: 34835 \\
\hline UAS-Rab7WT & Bloomington Drosophila Stock Center & BDSC: 23641 \\
\hline UAS-CP1EP & Bloomington Drosophila Stock Center & BDSC: 15957 \\
\hline UAS-Vha100-1EP & Bloomington Drosophila Stock Center & BDSC: 63269 \\
\hline UAS-TRPML & Bloomington Drosophila Stock Center & BDSC: 57372 \\
\hline UAS-Vha44EP & Bloomington Drosophila Stock Center & BDSC: 20140 \\
\hline UAS-VhaSFD & Bloomington Drosophila Stock Center & BDSC: 15758 \\
\hline$U A S-R a b 7^{D N}$ & Bloomington Drosophila Stock Center & BDSC: 9778 \\
\hline$U A S-C P 1^{R N A i}$ & Bloomington Drosophila Stock Center & BDSC: 32932 \\
\hline UAS-Vha100-1 ${ }^{\text {RAAi }}$ & Bloomington Drosophila Stock Center & BDSC: 26290 \\
\hline UAS-TRPML ${ }^{R N A i}$ & Bloomington Drosophila Stock Center & BDSC: 31294 \\
\hline UAS-Vha44 ${ }^{R N A i}$ & Bloomington Drosophila Stock Center & BDSC: 33884 \\
\hline UAS-VhaSFD ${ }^{R N A i}$ & Bloomington Drosophila Stock Center & BDSC: 40896 \\
\hline$w^{1118}$ & Bloomington Drosophila Stock Center & BDSC: 3605 \\
\hline Canton S & Bloomington Drosophila Stock Center & BDSC: 64349 \\
\hline \multicolumn{3}{|l|}{ Oligonucleotides (q-RT-PCR primers) } \\
\hline 5'- GCGCGGTTACTCTTTCACCA-3' & Integrated DNA Technologies & Actin forward \\
\hline
\end{tabular}


bioRxiv preprint doi: https://doi.org/10.1101/2020.06.26.173021; this version posted June 26, 2020. The copyright holder for this preprint (which was not certified by peer review) is the author/funder, who has granted bioRxiv a license to display the preprint in perpetuity. It is made available under aCC-BY 4.0 International license.

\begin{tabular}{|c|c|c|}
\hline 5'- ATGTCACGGACGATTTCACG-3' & Integrated DNA Technologies & Actin reverse \\
\hline 5'- GGGATCTAGCCACCATGGAG-3' & Integrated DNA Technologies & $\begin{array}{l}\mathrm{G}_{4} \mathrm{C}_{2} \text { repeats } \\
\text { forward (UAS-30R) }\end{array}$ \\
\hline 5'- TACCGTCGACTGCAGAGATTC-3' & Integrated DNA Technologies & $\begin{array}{l}\mathrm{G}_{4} \mathrm{C}_{2} \text { repeats } \\
\text { reverse (UAS-30R) }\end{array}$ \\
\hline 5'-AGTATCGGAGTAGATGTGCCAC-3' & Integrated DNA Technologies & mitf forward \\
\hline 5'- CGCTGAGATATTGCCTCACTTG-3' & Integrated DNA Technologies & mitf reverse \\
\hline 5'- TCTATGGCCCCTTCTTCGGA-3' & Integrated DNA Technologies & vha16-1 forward \\
\hline 5'- AATGGCAATGATACCCGCCA-3' & Integrated DNA Technologies & vha16-1 reverse \\
\hline 5'- CAAATATGGACGTGTCTTCGCT-3' & Integrated DNA Technologies & vha68-2 forward \\
\hline 5'- CCGGATCTCCGACAGTTACG-3' & Integrated DNA Technologies & vha68-2 reverse \\
\hline 5'- CGGGACTTTATCTCCCAGCC-3' & Integrated DNA Technologies & vha55 forward \\
\hline 5'-TGACCTCATCGAGAATGACCAG-3' & Integrated DNA Technologies & vha55 reverse \\
\hline 5'- TGGACTCGGAGTACCTGACC-3' & Integrated DNA Technologies & vha44 forward \\
\hline 5'- CGTCACGTTGAACAGGCAGTA-3' & Integrated DNA Technologies & vha44 reverse \\
\hline 5'- TGTTCCGTAGTGAGGAGATGG-3' & Integrated DNA Technologies & vha100-2 forward \\
\hline 5'- TCACGTTCACATTCAAGTCGC-3' & Integrated DNA Technologies & vha100-2 reverse \\
\hline 5'- GGTCAGTTCTACTTCCTCATTCG-3' & Integrated DNA Technologies & atg8a forward \\
\hline 5'- GATGTTCCTGGTACAGGGAGC-3' & Integrated DNA Technologies & atg8a reverse \\
\hline 5'- ACACGCCTCGAAACAGTGG-3' & Integrated DNA Technologies & atg9 forward \\
\hline 5'- TCAAGGTCCTCGATGTGGTTC-3' & Integrated DNA Technologies & atg9 reverse \\
\hline \multicolumn{3}{|l|}{ Recombinant DNA } \\
\hline pSF-CAG-Amp & Oxford Genetics & Cat\# 0G504 \\
\hline pSF-(G4C2)47-VFH & This paper & N/A \\
\hline pEGFP-(GA,GR, or PR)50 & Davide Trotti (Wen et. al 2014) & N/A \\
\hline mCherry-(GA,GR, or PR)50 & $\begin{array}{l}\text { This paper; adapted from } \\
\text { pEGFP-(GA,GR, or PR)50 (Wen } \\
\text { et. al 2014) }\end{array}$ & N/A \\
\hline \multicolumn{3}{|l|}{ Software and Algorithms } \\
\hline ImageJ & https://imagej.nih.gov/ij/ & $\mathrm{N} / \mathrm{A}$ \\
\hline GraphPad Prism 8 & $\begin{array}{l}\text { https://www.graphpad.com/scient } \\
\text { ific-software/prism/ }\end{array}$ & N/A \\
\hline Adobe Illustrator CC 2018 & $\begin{array}{l}\text { https://www.adobe.com/products } \\
\text { lillustrator }\end{array}$ & $\mathrm{N} / \mathrm{A}$ \\
\hline Image Pro Insight 9.1 & $\begin{array}{l}\text { http://www.mediacy.com/imagepr } \\
\underline{\mathrm{o}}\end{array}$ & $\mathrm{N} / \mathrm{A}$ \\
\hline WinWCP & $\begin{array}{l}\text { https://pureportal.strath.ac.uk/en/ } \\
\text { datasets/strathclyde- } \\
\text { electrophysiology-software- } \\
\text { winwcp-winedr }\end{array}$ & N/A \\
\hline
\end{tabular}

\section{Acknowledgments}

This work was supported by NINDS R01NS082563 (T.E.L), R01NS094239 (T.E.L and J.D.R.), F31 NS100401 (K.M.C.), ALSA (T.E.L., K.Z. and J.D.R.), and Target ALS (T.E.L., K.Z., J.D.R.,

5 and H.J.B.). K.M.C is a recipient of the P.E.O Scholar Award. H.J.B. is an Investigator of the

Howard Hughes Medical Institute. We thank Francesca Pignoni, Udai Pandey, Peng Jin, 
bioRxiv preprint doi: https://doi.org/10.1101/2020.06.26.173021; this version posted June 26, 2020. The copyright holder for this preprint

(which was not certified by peer review) is the author/funder, who has granted bioRxiv a license to display the preprint in perpetuity. It is made available under aCC-BY 4.0 International license.

Adrian Isaacs, Eric Baehrecke, Helmut Kramer, Francesca Pignoni, Gábor Juhász, Patrick

Dolph, L. Miguel Martins, the Bloomington Drosophila Stock Center (NIH P40ODO18537) and

Vienna Drosophila Research Center for Drosophila lines and/or antibodies and Shawn

Ferguson and Davide Trotti for cell lines and constructs. The Johns Hopkins NINDS

5 Multiphoton Imaging Core (NS050274) provided imaging equipment and expertise.

\section{Competing Interests}

The authors declare no competing interests.

\section{Author Contributions}

K.M.C., K.Z., and T.E.L. conceived the study and designed experiments; K.M.C., K.Z. K.R., H.S., and K.M. performed Drosophila and cell culture experiments and analyzed data. J.G. (supervised by J.D.R.) and K.M.C. performed human tissue experiments. M.S. and Z.Z. performed the TEM experiments. K.M.C., K.Z., H.J.B., and T.E.L. wrote and edited the

15 manuscript. T.E.L. supervised the project and acquired funding. 


\section{References}

Al-Sarraj, S., King, A., Troakes, C., Smith, B., Maekawa, S., Bodi, I., . . Shaw, C. E. (2011). p62 positive, TDP-43 negative, neuronal cytoplasmic and intranuclear inclusions in the cerebellum and hippocampus define the pathology of C9orf72-linked FTLD and MND/ALS. Acta Neuropathol, 122(6), 691-702. doi:10.1007/s00401-011-0911-2 10.1007/s00401-011-0911-2. Epub 2011 Nov 19.

Almeida, S., Gascon, E., Tran, H., Chou, H. J., Gendron, T. F., Degroot, S., ... Gao, F. B. (2013). Modeling key pathological features of frontotemporal dementia with C9ORF72 repeat expansion in iPSC-derived human neurons. Acta Neuropathologica, 126, 385399. doi:10.1007/s00401-013-1149-y

Balendra, R., \& Isaacs, A. M. (2018). C9orf72-mediated ALS and FTD: multiple pathways to disease. Nat Rev Neurol, 14(9), 544-558. doi:10.1038/s41582-018-0047-2

Bharadwaj, R., Cunningham, K. M., Zhang, K., \& Lloyd, T. E. (2016). FIG4 regulates lysosome membrane homeostasis independent of phosphatase function. Hum Mol Genet, 25(4), 681-692. doi:10.1093/hmg/ddv505

Boeynaems, S., Bogaert, E., Michiels, E., Gijselinck, I., Sieben, A., Jovicic, A., . . Van Den Bosch, L. (2016). Drosophila screen connects nuclear transport genes to DPR pathology in c9ALS/FTD. Sci Rep, 6, 20877. doi:10.1038/srep20877

Bouche, V., Espinosa, A. P., Leone, L., Sardiello, M., Ballabio, A., \& Botas, J. (2016). Drosophila Mitf regulates the V-ATPase and the lysosomal-autophagic pathway. Autophagy, 12(3), 484-498. doi:10.1080/15548627.2015.1134081

Chen, Y., Liu, H., Guan, Y., Wang, Q., Zhou, F., Jie, L., . . Wang, X. (2015). The altered autophagy mediated by TFEB in animal and cell models of amyotrophic lateral sclerosis. Am J Transl Res, 7(9), 1574-1587.

Chou, C. C., Zhang, Y., Umoh, M. E., Vaughan, S. W., Lorenzini, I., Liu, F., . . Rossoll, W. (2018). TDP-43 pathology disrupts nuclear pore complexes and nucleocytoplasmic transport in ALS/FTD. Nat Neurosci, 21(2), 228-239. doi:10.1038/s41593-017-0047-3

Chouhan, A. K., Guo, C., Hsieh, Y. C., Ye, H., Senturk, M., Zuo, Z., . . Shulman, J. M. (2016). Uncoupling neuronal death and dysfunction in Drosophila models of neurodegenerative disease. Acta neuropathologica communications. doi:10.1186/s40478-016-0333-4

Cipolat Mis, M. S., Brajkovic, S., Frattini, E., Di Fonzo, A., \& Corti, S. (2016). Autophagy in motor neuron disease: Key pathogenetic mechanisms and therapeutic targets. Mol Cell Neurosci, 72, 84-90. doi:10.1016/j.mcn.2016.01.012

Cirulli, E. T., Lasseigne, B. N., Petrovski, S., Sapp, P. C., Dion, P. A., Leblond, C. S., . . . Goldstein, D. B. (2015). Exome sequencing in amyotrophic lateral sclerosis identifies risk genes and pathways. Science, 347(6229), 1436-1441. doi:10.1126/science.aaa3650

40 Cooper-Knock, J., Hewitt, C., Highley, J. R., Brockington, A., Milano, A., Man, S., ... Shaw, P. J. (2012). Clinico-pathological features in amyotrophic lateral sclerosis with expansions in C9ORF72. Brain, 135(Pt 3), 751-764. doi:10.1093/brain/awr365

Cortes, C. J., \& La Spada, A. R. (2019). TFEB dysregulation as a driver of autophagy dysfunction in neurodegenerative disease: Molecular mechanisms, cellular processes, and emerging therapeutic opportunities. Neurobiol Dis, 122, 83-93. doi:10.1016/j.nbd.2018.05.012

Decressac, M., Mattsson, B., Weikop, P., Lundblad, M., Jakobsson, J., \& Bjorklund, A. (2013). TFEB-mediated autophagy rescues midbrain dopamine neurons from alpha-synuclein toxicity. Proc Natl Acad Sci U S A, 110(19), E1817-1826. doi:10.1073/pnas.1305623110 
DeJesus-Hernandez, M., Mackenzie, I. R., Boeve, B. F., Boxer, A. L., Baker, M., Rutherford, N. J., . . Rademakers, R. (2011). Expanded GGGGCC hexanucleotide repeat in noncoding region of C9ORF72 causes chromosome 9p-linked FTD and ALS. Neuron, 72(2), 245-256. doi:10.1016/j.neuron.2011.09.011

5 Donde, A., Sun, M., Jeong, Y. H., Wen, X., Ling, J., Lin, S., . . . Wong, P. C. (2020). Upregulation of ATG7 attenuates motor neuron dysfunction associated with depletion of TARDBP/TDP-43. Autophagy, 16(4), 672-682. doi:10.1080/15548627.2019.1635379

Donnelly, C. J., Zhang, P. W., Pham, J. T., Haeusler, A. R., Mistry, N. A., Vidensky, S., . . Rothstein, J. D. (2013). RNA toxicity from the ALS/FTD C9ORF72 expansion is mitigated by antisense intervention. Neuron, 80(2), 415-428. doi:10.1016/j.neuron.2013.10.015

Eftekharzadeh, B., Daigle, J. G., Kapinos, L. E., Coyne, A., Schiantarelli, J., Carlomagno, Y., . . . Hyman, B. T. (2018). Tau Protein Disrupts Nucleocytoplasmic Transport in Alzheimer's Disease. Neuron, 99(5), 925-940 e927. doi:10.1016/j.neuron.2018.07.039

15 Evans, C. S., \& Holzbaur, E. L. F. (2019). Autophagy and mitophagy in ALS. Neurobiol Dis, 122, 35-40. doi:10.1016/j.nbd.2018.07.005

Farg, M. A., Sundaramoorthy, V., Sultana, J. M., Yang, S., Atkinson, R. A., Levina, V., . . . Atkin, J. D. (2014). C9ORF72, implicated in amytrophic lateral sclerosis and frontotemporal dementia, regulates endosomal trafficking. Hum Mol Genet, 23(13), 3579-3595. doi:10.1093/hmg/ddu068

Fraldi, A., Klein, A. D., Medina, D. L., \& Settembre, C. (2016). Brain Disorders Due to Lysosomal Dysfunction. Annu Rev Neurosci, 39, 277-295. doi:10.1146/annurev-neuro070815-014031

Freibaum, B. D., Lu, Y., Lopez-Gonzalez, R., Kim, N. C., Almeida, S., Lee, K. H., . . Taylor, J. P. (2015). GGGGCC repeat expansion in C9orf72 compromises nucleocytoplasmic transport. Nature, 525(7567), 129-133. doi:10.1038/nature14974

Gao, F. B., Almeida, S., \& Lopez-Gonzalez, R. (2017). Dysregulated molecular pathways in amyotrophic lateral sclerosis-frontotemporal dementia spectrum disorder. EMBO J, 36(20), 2931-2950. doi:10.15252/embj.201797568

Gasset-Rosa, F., Chillon-Marinas, C., Goginashvili, A., Atwal, R. S., Artates, J. W., Tabet, R., . .. Lagier-Tourenne, C. (2017). Polyglutamine-Expanded Huntingtin Exacerbates AgeRelated Disruption of Nuclear Integrity and Nucleocytoplasmic Transport. Neuron, 94(1), 48-57 e44. doi:10.1016/j.neuron.2017.03.027

Gasset-Rosa, F., Lu, S., Yu, H., Chen, C., Melamed, Z., Guo, L., . . Cleveland, D. W. (2019). Cytoplasmic TDP-43 De-mixing Independent of Stress Granules Drives Inhibition of Nuclear Import, Loss of Nuclear TDP-43, and Cell Death. Neuron, 102(2), 339-357 e337. doi:10.1016/j.neuron.2019.02.038

Goode, A., Butler, K., Long, J., Cavey, J., Scott, D., Shaw, B., . . . Layfield, R. (2016). Defective recognition of LC3B by mutant SQSTM1/p62 implicates impairment of autophagy as a pathogenic mechanism in ALS-FTLD. Autophagy, 12(7), 1094-1104. doi:10.1080/15548627.2016.1170257

Goodman, L. D., Prudencio, M., Kramer, N. J., Martinez-Ramirez, L. F., Srinivasan, A. R., Lan, M., . . Bonini, N. M. (2019). Toxic expanded GGGGCC repeat transcription is mediated by the PAF1 complex in C9orf72-associated FTD. Nat Neurosci, 22(6), 863-874. doi:10.1038/s41593-019-0396-1

Grima, J. C., Daigle, J. G., Arbez, N., Cunningham, K. C., Zhang, K., Ochaba, J., . . . Rothstein, J. D. (2017). Mutant Huntingtin Disrupts the Nuclear Pore Complex. Neuron, 94(1), 93-107 e106. doi:10.1016/j.neuron.2017.03.023

Hallsson, J. H., Haflidadottir, B. S., Stivers, C., Odenwald, W., Arnheiter, H., Pignoni, F., \& Steingrimsson, E. (2004). The basic helix-loop-helix leucine zipper transcription factor 
Mitf is conserved in Drosophila and functions in eye development. Genetics, 167(1), 233-241. doi:10.1534/genetics.167.1.233

Hariri, M., Millane, G., Guimond, M. P., Guay, G., Dennis, J. W., \& Nabi, I. R. (2000). Biogenesis of multilamellar bodies via autophagy. Mol Biol Cell, 11(1), 255-268. doi:10.1091/mbc.11.1.255

Ji, Y. J., Ugolino, J., Brady, N. R., Hamacher-Brady, A., \& Wang, J. (2017). Systemic deregulation of autophagy upon loss of ALS- and FTD-linked C9orf72. Autophagy, 13(7), 1254-1255. doi:10.1080/15548627.2017.1299312

Jovicic, A., Mertens, J., Boeynaems, S., Bogaert, E., Chai, N., Yamada, S. B., . . Gitler, A. D. (2015). Modifiers of C9orf72 dipeptide repeat toxicity connect nucleocytoplasmic transport defects to FTD/ALS. Nat Neurosci, 18(9), 1226-1229. doi:10.1038/nn.4085

Kinser, R. D., \& Dolph, P. J. (2012). Cathepsin proteases mediate photoreceptor cell degeneration in Drosophila. Neurobiol Dis, 46(3), 655-662. doi:10.1016/j.nbd.2012.03.004

15 Klionsky, D. J., Abdelmohsen, K., Abe, A., Abedin, M. J., Abeliovich, H., Acevedo Arozena, A., ... Zughaier, S. M. (2016). Guidelines for the use and interpretation of assays for monitoring autophagy (3rd edition). Autophagy, 12(1), 1-222. doi:10.1080/15548627.2015.1100356

Komatsu, M., Waguri, S., Koike, M., Sou, Y. S., Ueno, T., Hara, T., . . . Tanaka, K. (2007). Homeostatic levels of p62 control cytoplasmic inclusion body formation in autophagydeficient mice. Cell, 131(6), 1149-1163. doi:10.1016/j.cell.2007.10.035

Korolchuk, V. I., Menzies, F. M., \& Rubinsztein, D. C. (2010). Mechanisms of cross-talk between the ubiquitin-proteasome and autophagy-lysosome systems. FEBS Lett, 584(7), 1393-1398. doi:10.1016/j.febslet.2009.12.047

Kramer, N. J., Carlomagno, Y., Zhang, Y. J., Almeida, S., Cook, C. N., Gendron, T. F., . . Gitler, A. D. (2016). Spt4 selectively regulates the expression of C9orf72 sense and antisense mutant transcripts. Science, 353(6300), 708-712. doi:10.1126/science.aaf7791

Kwon, I., Xiang, S., Kato, M., Wu, L., Theodoropoulos, P., Wang, T., . . McKnight, S. L. (2014). Poly-dipeptides encoded by the C9orf72 repeats bind nucleoli, impede RNA biogenesis, and kill cells. Science, 345(6201), 1139-1145. doi:10.1126/science.1254917

Le Ber, I., Camuzat, A., Guerreiro, R., Bouya-Ahmed, K., Bras, J., Nicolas, G., . . Genetic Research Network on, F. F.-A. (2013). SQSTM1 mutations in French patients with frontotemporal dementia or frontotemporal dementia with amyotrophic lateral sclerosis. JAMA Neurol, 70(11), 1403-1410. doi:10.1001/jamaneurol.2013.3849

Lee, C. W., Wilfling, F., Ronchi, P., Allegretti, M., Mosalaganti, S., Jentsch, S., .. . Pfander, B. (2020). Selective autophagy degrades nuclear pore complexes. Nat Cell Biol, 22(2), 159-166. doi:10.1038/s41556-019-0459-2

Levine, B., \& Kroemer, G. (2008). Autophagy in the pathogenesis of disease. Cell, 132(1), 2742. doi:10.1016/j.cell.2007.12.018

Li, L., Friedrichsen, H. J., Andrews, S., Picaud, S., Volpon, L., Ngeow, K., . . Goding, C. R. (2018). A TFEB nuclear export signal integrates amino acid supply and glucose availability. Nat Commun, 9(1), 2685. doi:10.1038/s41467-018-04849-7

Lin, G., Mao, D., \& Bellen, H. J. (2017). Amyotrophic Lateral Sclerosis Pathogenesis Converges on Defects in Protein Homeostasis Associated with TDP-43 Mislocalization and Proteasome-Mediated Degradation Overload.

Ling, S. C., Polymenidou, M., \& Cleveland, D. W. (2013). Converging mechanisms in ALS and FTD: disrupted RNA and protein homeostasis. Neuron, 79(3), 416-438. doi:10.1016/j.neuron.2013.07.033 

available under aCC-BY 4.0 International license.

Liu, Y., Pattamatta, A., Zu, T., Reid, T., Bardhi, O., Borchelt, D. R., . . Ranum, L. P. (2016). C9orf72 BAC Mouse Model with Motor Deficits and Neurodegenerative Features of ALS/FTD. Neuron, 90(3), 521-534. doi:10.1016/j.neuron.2016.04.005

Mackenzie, I. R., Frick, P., \& Neumann, M. (2014). The neuropathology associated with repeat expansions in the C9ORF72 gene. Acta Neuropathol, 127(3), 347-357. doi:10.1007/s00401-013-1232-4

Mao, D., Lin, G., Tepe, B., Zuo, Z., Tan, K. L., Senturk, M., . . Bellen, H. J. (2019). VAMP associated proteins are required for autophagic and lysosomal degradation by promoting a Ptdlns4P-mediated endosomal pathway. Autophagy, 15(7), 1214-1233. doi:10.1080/15548627.2019.1580103

Martini-Stoica, H., Xu, Y., Ballabio, A., \& Zheng, H. (2016). The Autophagy-Lysosomal Pathway in Neurodegeneration: A TFEB Perspective. Trends Neurosci, 39(4), 221-234. doi:10.1016/j.tins.2016.02.002

Mauvezin, C., Ayala, C., Braden, C. R., Kim, J., \& Neufeld, T. P. (2014). Assays to monitor autophagy in Drosophila. Methods, 68(1), 134-139. doi:10.1016/j.ymeth.2014.03.014

Mizielinska, S., \& Isaacs, A. M. (2014). C9orf72 amyotrophic lateral sclerosis and frontotemporal dementia: gain or loss of function? Curr Opin Neurol, 27(5), 515-523. doi:10.1097/WCO.0000000000000130

Mori, K., Weng, S. M., Arzberger, T., May, S., Rentzsch, K., Kremmer, E., . . Edbauer, D. (2013). The C9orf72 GGGGCC repeat is translated into aggregating dipeptide-repeat proteins in FTLD/ALS. Science, 339(6125), 1335-1338. doi:10.1126/science.1232927

O'Rourke, J. G., Bogdanik, L., Muhammad, A., Gendron, T. F., Kim, K. J., Austin, A., . . . Baloh, R. H. (2015). C9orf72 BAC Transgenic Mice Display Typical Pathologic Features of ALS/FTD. Neuron, 88(5), 892-901. doi:10.1016/j.neuron.2015.10.027

Ortega, J. A., Daley, E. L., Kour, S., Samani, M., Tellez, L., Smith, H. S., . . Kiskinis, E. (2020). Nucleocytoplasmic Proteomic Analysis Uncovers eRF1 and Nonsense-Mediated Decay as Modifiers of ALS/FTD C9orf72 Toxicity. Neuron, 106(1), 90-107.e113. doi:10.1016/j.neuron.2020.01.020

Palmieri, M., Impey, S., Kang, H., di Ronza, A., Pelz, C., Sardiello, M., \& Ballabio, A. (2011). Characterization of the CLEAR network reveals an integrated control of cellular clearance pathways. Hum Mol Genet, 20(19), 3852-3866. doi:10.1093/hmg/ddr306

Parkinson-Lawrence, E. J., Shandala, T., Prodoehl, M., Plew, R., Borlace, G. N., \& Brooks, D. A. (2010). Lysosomal storage disease: revealing lysosomal function and physiology. Physiology (Bethesda), 25(2), 102-115. doi:10.1152/physiol.00041.2009

Parr, C., Carzaniga, R., Gentleman, S. M., Van Leuven, F., Walter, J., \& Sastre, M. (2012). Glycogen synthase kinase 3 inhibition promotes lysosomal biogenesis and autophagic degradation of the amyloid-beta precursor protein. Mol Cell Biol, 32(21), 4410-4418. doi:10.1128/MCB.00930-12

Polito, V. A., Li, H., Martini-Stoica, H., Wang, B., Yang, L., Xu, Y., . . Z Zheng, H. (2014). Selective clearance of aberrant tau proteins and rescue of neurotoxicity by transcription factor EB. EMBO Mol Med, 6(9), 1142-1160. doi:10.15252/emmm.201303671

Pulipparacharuvil, S., Akbar, M. A., Ray, S., Sevrioukov, E. A., Haberman, A. S., Rohrer, J., \& Kramer, H. (2005). Drosophila Vps16A is required for trafficking to lysosomes and biogenesis of pigment granules. J Cell Sci, 118(Pt 16), 3663-3673. doi:10.1242/jcs.02502

Ramesh, N., \& Pandey, U. B. (2017). Autophagy Dysregulation in ALS: When Protein Aggregates Get Out of Hand. Front Mol Neurosci, 10, 263. doi:10.3389/fnmol.2017.00263

Renton, A. E., Majounie, E., Waite, A., Simon-Sanchez, J., Rollinson, S., Gibbs, J. R., . . . Traynor, B. J. (2011). A hexanucleotide repeat expansion in C9ORF72 is the cause of 
chromosome 9p21-linked ALS-FTD. Neuron, 72(2), 257-268.

doi:10.1016/j.neuron.2011.09.010

Roczniak-Ferguson, A., Petit, C. S., Froehlich, F., Qian, S., Ky, J., Angarola, B., ... Ferguson, S. M. (2012). The transcription factor TFEB links mTORC1 signaling to transcriptional control of lysosome homeostasis. Sci Signal, 5(228), ra42. doi:10.1126/scisignal.2002790

Saitoh, Y., Fujikake, N., Okamoto, Y., Popiel, H. A., Hatanaka, Y., Ueyama, M., . . Nagai, Y. (2015). p62 plays a protective role in the autophagic degradation of polyglutamine protein oligomers in polyglutamine disease model flies. J Biol Chem, 290(3), 1442-1453. doi:10.1074/jbc.M114.590281

Sardiello, M., Palmieri, M., di Ronza, A., Medina, D. L., Valenza, M., Gennarino, V. A., .. . Ballabio, A. (2009). A gene network regulating lysosomal biogenesis and function. Science, 325(5939), 473-477. doi:10.1126/science.1174447

Sasaki, S. (2011). Autophagy in spinal cord motor neurons in sporadic amyotrophic lateral sclerosis. J Neuropathol Exp Neurol, 70(5), 349-359. doi:10.1097/NEN.0b013e3182160690

Sellier, C., Campanari, M. L., Julie Corbier, C., Gaucherot, A., Kolb-Cheynel, I., OuladAbdelghani, M., . . . Charlet-Berguerand, N. (2016). Loss of C9ORF72 impairs autophagy and synergizes with polyQ Ataxin-2 to induce motor neuron dysfunction and cell death. EMBO J, 35(12), 1276-1297. doi:10.15252/embj.201593350

Senturk, M., Lin, G., Zuo, Z., Mao, D., Watson, E., Mikos, A. G., \& Bellen, H. J. (2019). Ubiquilins regulate autophagic flux through mTOR signalling and lysosomal acidification. Nat Cell Biol, 21(3), 384-396. doi:10.1038/s41556-019-0281-x

Settembre, C., Di Malta, C., Polito, V. A., Garcia Arencibia, M., Vetrini, F., Erdin, S., ... Ballabio, A. (2011). TFEB links autophagy to lysosomal biogenesis. Science, 332(6036), 1429-1433. doi:10.1126/science.1204592

Shi, K. Y., Mori, E., Nizami, Z. F., Lin, Y., Kato, M., Xiang, S., . . McKnight, S. L. (2017). Toxic PRn poly-dipeptides encoded by the C9orf72 repeat expansion block nuclear import and export. Proc Natl Acad Sci U S A, 114(7), E1111-E1117. doi:10.1073/pnas.1620293114

Shi, Y., Lin, S., Staats, K. A., Li, Y., Chang, W. H., Hung, S. T., . . Ichida, J. K. (2018). Haploinsufficiency leads to neurodegeneration in C9ORF72 ALS/FTD human induced motor neurons. Nat Med, 24(3), 313-325. doi:10.1038/nm.4490

Silvestrini, M. J., Johnson, J. R., Kumar, A. V., Thakurta, T. G., Blais, K., Neill, Z. A., . . Lapierre, L. R. (2018). Nuclear Export Inhibition Enhances HLH-30/TFEB Activity, Autophagy, and Lifespan. Cell Rep, 23(7), 1915-1921. doi:10.1016/j.celrep.2018.04.063

Solomon, D. A., Stepto, A., Au, W. H., Adachi, Y., Diaper, D. C., Hall, R., . . Hirth, F. (2018). A feedback loop between dipeptide-repeat protein, TDP-43 and karyopherin-alpha mediates C9orf72-related neurodegeneration. Brain, 141(10), 2908-2924. doi:10.1093/brain/awy241

Song, W., Wang, F., Savini, M., Ake, A., di Ronza, A., Sardiello, M., \& Segatori, L. (2013). TFEB regulates lysosomal proteostasis. Hum Mol Genet, 22(10), 1994-2009. doi:10.1093/hmg/ddt052

Sullivan, P. M., Zhou, X., Robins, A. M., Paushter, D. H., Kim, D., Smolka, M. B., \& Hu, F. (2016). The ALS/FTLD associated protein C9orf72 associates with SMCR8 and WDR41 to regulate the autophagy-lysosome pathway. Acta Neuropathol Commun, 4(1), 51. doi:10.1186/s40478-016-0324-5

Teyssou, E., Takeda, T., Lebon, V., Boillee, S., Doukoure, B., Bataillon, G., . . Millecamps, S. (2013). Mutations in SQSTM1 encoding p62 in amyotrophic lateral sclerosis: genetics and neuropathology. Acta Neuropathol, 125(4),511-522.doi:10.1007/s00401-013-10900 
Thomas, M., Alegre-Abarrategui, J., \& Wade-Martins, R. (2013). RNA dysfunction and aggrephagy at the centre of an amyotrophic lateral sclerosis/frontotemporal dementia disease continuum. Brain, 136(Pt 5), 1345-1360. doi:10.1093/brain/awt030

Torra, A., Parent, A., Cuadros, T., Rodriguez-Galvan, B., Ruiz-Bronchal, E., Ballabio, A., . . . Bove, J. (2018). Overexpression of TFEB Drives a Pleiotropic Neurotrophic Effect and Prevents Parkinson's Disease-Related Neurodegeneration. Mol Ther, 26(6), 1552-1567. doi:10.1016/j.ymthe.2018.02.022

Tran, H., Almeida, S., Moore, J., Gendron, T. F., Chalasani, U., Lu, Y., . . . Gao, F. B. (2015). Differential Toxicity of Nuclear RNA Foci versus Dipeptide Repeat Proteins in a Drosophila Model of C9ORF72 FTD/ALS. Neuron, 87(6), 1207-1214. doi:10.1016/j.neuron.2015.09.015

Ugolino, J., Ji, Y. J., Conchina, K., Chu, J., Nirujogi, R. S., Pandey, A., . . Wang, J. (2016). Loss of C9orf72 Enhances Autophagic Activity via Deregulated mTOR and TFEB Signaling. PLoS Genet, 12(11), e1006443. doi:10.1371/journal.pgen.1006443

Vodicka, P., Chase, K., Iuliano, M., Tousley, A., Valentine, D. T., Sapp, E., .. . DiFiglia, M. (2016). Autophagy Activation by Transcription Factor EB (TFEB) in Striatum of HDQ175/Q7 Mice. J Huntingtons Dis, 5(3), 249-260. doi:10.3233/JHD-160211

Walker, C., Herranz-Martin, S., Karyka, E., Liao, C., Lewis, K., Elsayed, W., . . . El-Khamisy, S. F. (2017). C9orf72 expansion disrupts ATM-mediated chromosomal break repair. Nat Neurosci, 20(9), 1225-1235. doi:10.1038/nn.4604

Wang, H., Wang, R., Xu, S., \& Lakshmana, M. K. (2016). Transcription Factor EB Is Selectively Reduced in the Nuclear Fractions of Alzheimer's and Amyotrophic Lateral Sclerosis Brains. Neurosci J, 2016, 4732837. doi:10.1155/2016/4732837

Weaver, T. E., Na, C. L., \& Stahlman, M. (2002). Biogenesis of lamellar bodies, lysosomerelated organelles involved in storage and secretion of pulmonary surfactant. Semin Cell Dev Biol, 13(4), 263-270. doi:10.1016/s1084952102000551

Webster, C. P., Smith, E. F., Bauer, C. S., Moller, A., Hautbergue, G. M., Ferraiuolo, L., ... De Vos, K. J. (2016). The C9orf72 protein interacts with Rab1a and the ULK1 complex to regulate initiation of autophagy. EMBO J, 35(15), 1656-1676. doi:10.15252/embj.201694401

Woerner, A. C., Frottin, F., Hornburg, D., Feng, L. R., Meissner, F., Patra, M., .. . Hipp, M. S. (2016). Cytoplasmic protein aggregates interfere with nucleocytoplasmic transport of protein and RNA. Science, 351(6269), 173-176. doi:10.1126/science.aad2033

Xu, Z., Poidevin, M., Li, X., Li, Y., Shu, L., Nelson, D. L., . . Jin, P. (2013). Expanded GGGGCC repeat RNA associated with amyotrophic lateral sclerosis and frontotemporal dementia causes neurodegeneration. Proc Natl Acad Sci U S A, 110(19), 7778-7783. doi:10.1073/pnas.1219643110

Yang, M., Liang, C., Swaminathan, K., Herrlinger, S., Lai, F., Shiekhattar, R., \& Chen, J. F. (2016). A C9ORF72/SMCR8-containing complex regulates ULK1 and plays a dual role in autophagy. Sci Adv, 2(9), e1601167. doi:10.1126/sciadv.1601167

Zhang, K., Daigle, J. G., Cunningham, K. M., Coyne, A. N., Ruan, K., Grima, J. C., . . Lloyd, T. E. (2018). Stress Granule Assembly Disrupts Nucleocytoplasmic Transport. Cell, 173(4), 958-971 e917. doi:10.1016/j.cell.2018.03.025

Zhang, K., Donnelly, C. J., Haeusler, A. R., Grima, J. C., Machamer, J. B., Steinwald, P., . ., Lloyd, T.E., Rothstein, J. D. (2015). The C9orf72 repeat expansion disrupts nucleocytoplasmic transport. Nature, 525(7567), 56-61. doi:10.1038/nature14973

Zhang, T., Zhou, Q., Ogmundsdottir, M. H., Moller, K., Siddaway, R., Larue, L., . . . Pignoni, F. (2015). Mitf is a master regulator of the v-ATPase, forming a control module for cellular homeostasis with v-ATPase and TORC1. J Cell Sci, 128(15), 2938-2950. doi:10.1242/jcs.173807 
bioRxiv preprint doi: https://doi org/10.1101/2020.06.26.173021; this version posted June 26, 2020. The copyright holder for this preprint (which was not certified by peer review) is the author/funder, who has granted bioRxiv a license to display the preprint in perpetuity. It is made available under aCC-BY 4.0 International license.

Zhang, Y. J., Gendron, T. F., Grima, J. C., Sasaguri, H., Jansen-West, K., Xu, Y. F., . . . Petrucelli, L. (2016). C9ORF72 poly(GA) aggregates sequester and impair HR23 and nucleocytoplasmic transport proteins. Nat Neurosci, 19(5), 668-677. doi:10.1038/nn.4272

5 Zhu, Q., Jiang, J., Gendron, T. F., McAlonis-Downes, M., Jiang, L., Taylor, A., ... Cleveland, D. W. (2020). Reduced C9ORF72 function exacerbates gain of toxicity from ALS/FTDcausing repeat expansion in C9orf72. Nat Neurosci. doi:10.1038/s41593-020-0619-5 

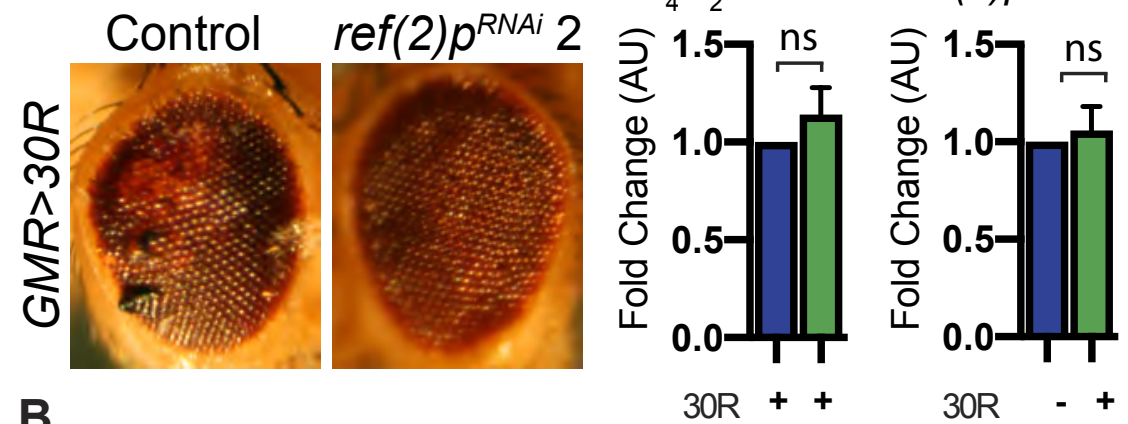

B

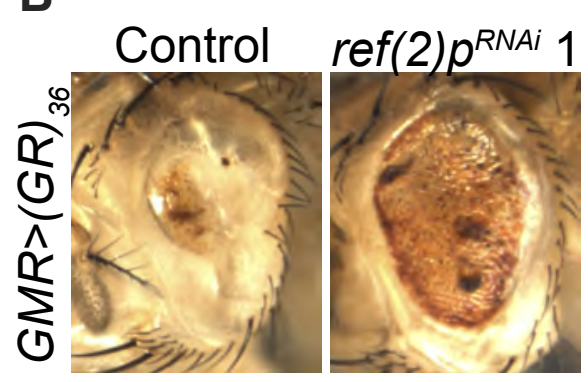

\section{$30 \mathrm{R}++$}

GMR-Gal4

Act-Gal4

\section{Supplemental Figure 1 (Related to Figure 1): Ref(2)p/p62 genetically modifies}

G4C2-HRE. A) Control and a second $\operatorname{Ref}(2) p^{R N A i}$ line $\left(\operatorname{Ref}(2) p^{H M S 00938}\right.$, see methods for detailed line information) in $G M R>30 R$ expressing Drosophila eyes at 15 days of age.

B) GMR-Gal4 expressing an alternate codon poly-GR $R_{36}$ alone or co-expressed with $\operatorname{Ref}(2) p^{R N A i}\left(\operatorname{Ref}(2) p^{H M S 00551}\right)$ at 15 days of age. D) Levels of G4C2 RNA measured by qPCR (See methods) in Drosophila expressing 30R under the control of GMR-Ga/4 either with or without $\operatorname{Ref}(2) p R N A i, \mathrm{n}=3$, Student's t-test. E) Levels of $\operatorname{Ref(2)p~RNA~in~}$ Drosophila larva expressing 30R ubiquitously under control of the Actin-Ga/4, $\mathrm{n}=5$, Student's t-test. Data represented are mean \pm SEM. n.s., not significant. 
bioRxiv preprint doi: https://doi.org/10.1101/2020.06.26.173021; this version posted June 26, 2020. The copyright holder for this preprint

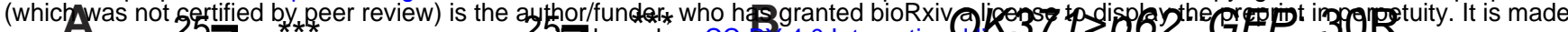

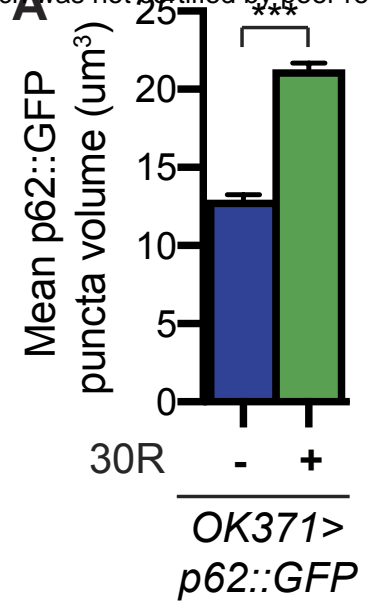

C

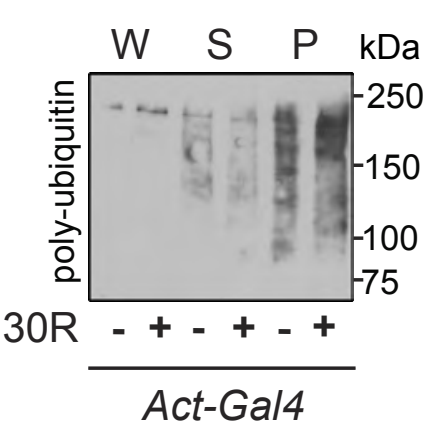

D Act/+

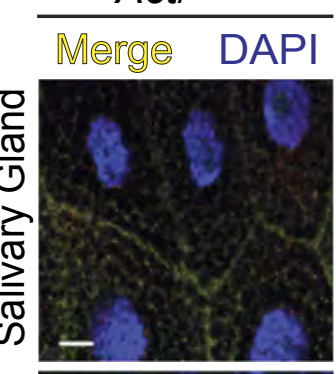

$\stackrel{0}{3}$

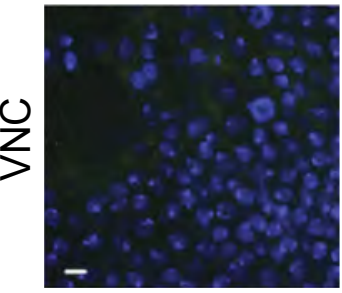

E

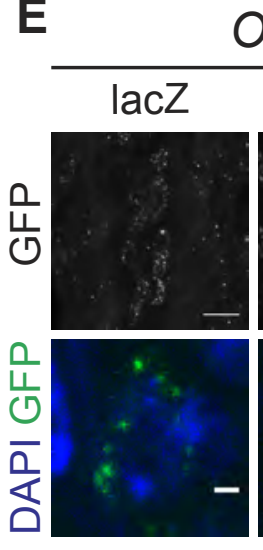

OK371>p62::GFP
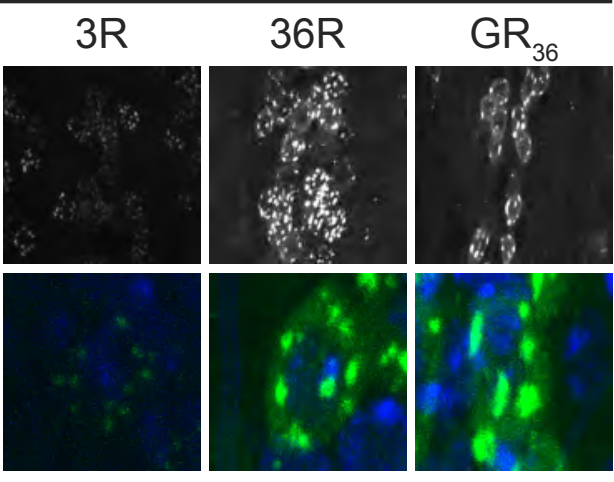

G

OK371>mCherry::Atg8
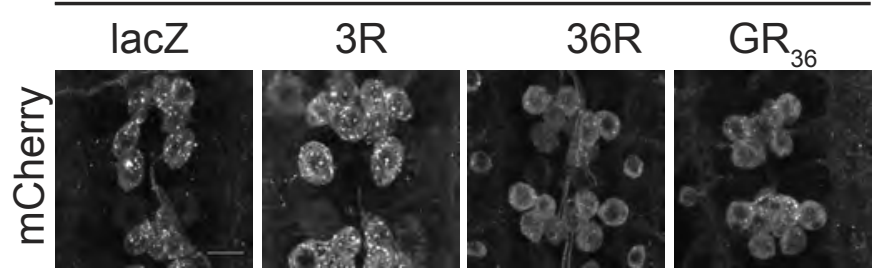

है

\section{Supplemental Figure 2}

Cunningham et al.

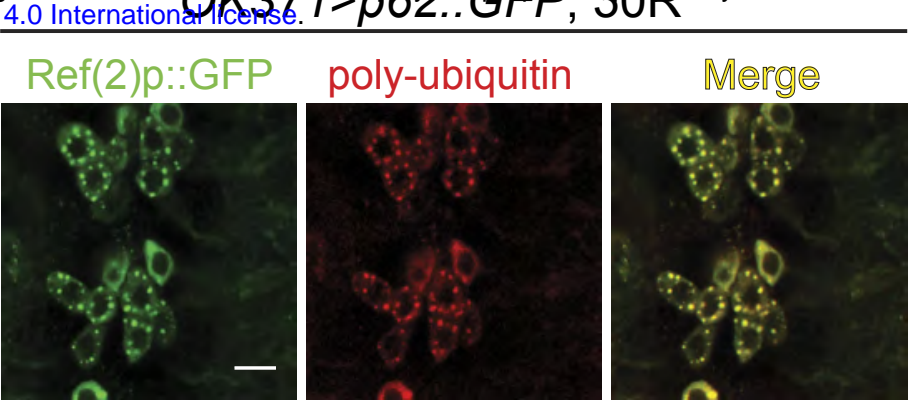

Act>30R
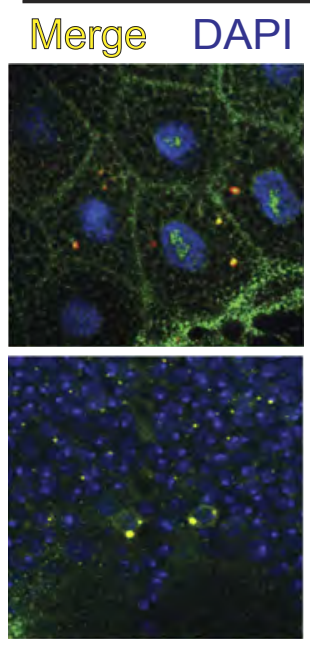

$F$
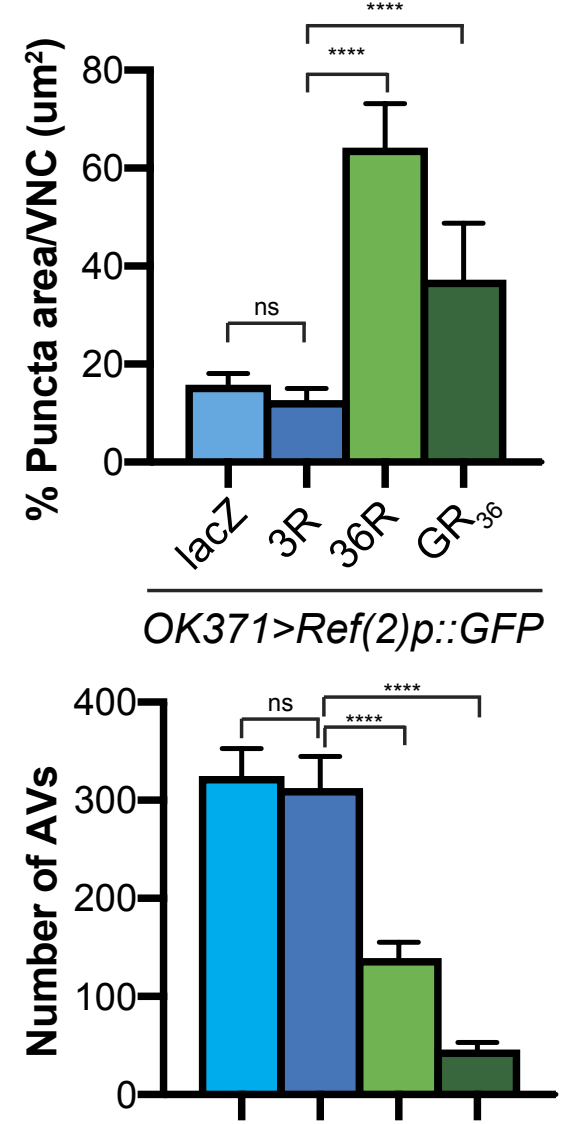

$$
\text { OK371>mCherry::Atg8 }
$$




\section{Supplemental Figure 2 (Related to Figure 2): p62::GFP aggregates in C9-ALS fly} models and co-localizes with poly-ubiquitin. A) Quantification of mean intensity and mean volume of p62::GFP puncta in OK371/+ or OK371>30R motor neuron cell bodies, $\mathrm{n}=5$ Student's t-test with Welch's correction ${ }^{* *} \mathrm{p}<.0001$. B) Example image of Drosophila OK371-Gal4 motor neurons expressing 30R; p62::GFP (green) co-stained with an anti-poly-ubiquitin (red). C) Western blot of anti-poly-ubiquitin showing the whole (W), supernatant (S) and pellet (P) fractions of Drosophila L3 larvae ubiquitously expressing 30R under the control of Act-Gal4. D) Images of Drosophila L3 larvae ActGal4/+ or Act>30R ubiquitously expressing G4C2 repeats showing anti-Ref(2)p (red) and anti-ubiquitin (green) staining in the larval salivary gland (top), ventral nerve cord (VNC) (bottom). E) OK371>p62::GFP showing the VNC (top) or a representative cell body (bottom) with two control (UAS-lacZ or UAS-3R) and two alternate C9-ALS model lines, one expressing a $\mathrm{G} 4 \mathrm{C} 2$ repeat expansion (UAS-36R) and another expressing the alternate codon arginine dipeptide GR $\left(U A S-G R_{36}\right)$. F) Quantification of the total GFP+ area of p62::GFP positive puncta in A). G) OK371>mCherry::Atg8 showing the VNC (top) or a representative cell body (bottom, scale bar represents 1um) with two control overexpression (UAS-lacZ or UAS-3R) and two alternate C9-ALS model lines, one expressing a G4C2 repeat expansion (UAS-36R) and another expressing the alternate codon arginine dipeptide GR (UAS-GR $\left.R_{36}\right)$. H) Quantification of mCherry:Atg8 puncta in the motor neuron cell bodies in $(G)$. Data represented are mean \pm SEM. Scale bar represents $10 \mu \mathrm{m}$. n.s., not significant, ${ }^{* * *} p<0.001,{ }^{* * *} p<0.0001$. 
bioRxiv preprint doi: https://doi.org/10.1101/2020.06.26.173021; this version posted June 26, 2020. The copyright holder for this preprint (which was not certified by peer review) is the author/funder, who has granted bioBxiv a license to display the preprint in perpetuity. It is made A Elavyalisunder aCC-BY 4.0 Internatibay íbónse.

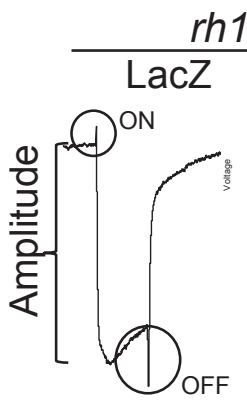

$n=10$
1-Gal4

$\frac{r h 1-\mathrm{Gal} 4}{\mathrm{LaCZ} 30 \mathrm{R}}$

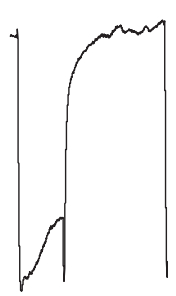

$\mathrm{n}=10$

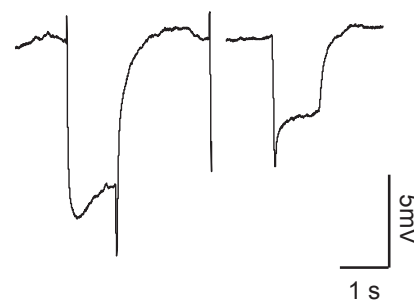

$\mathrm{n}=6$

$\mathrm{n}=7$
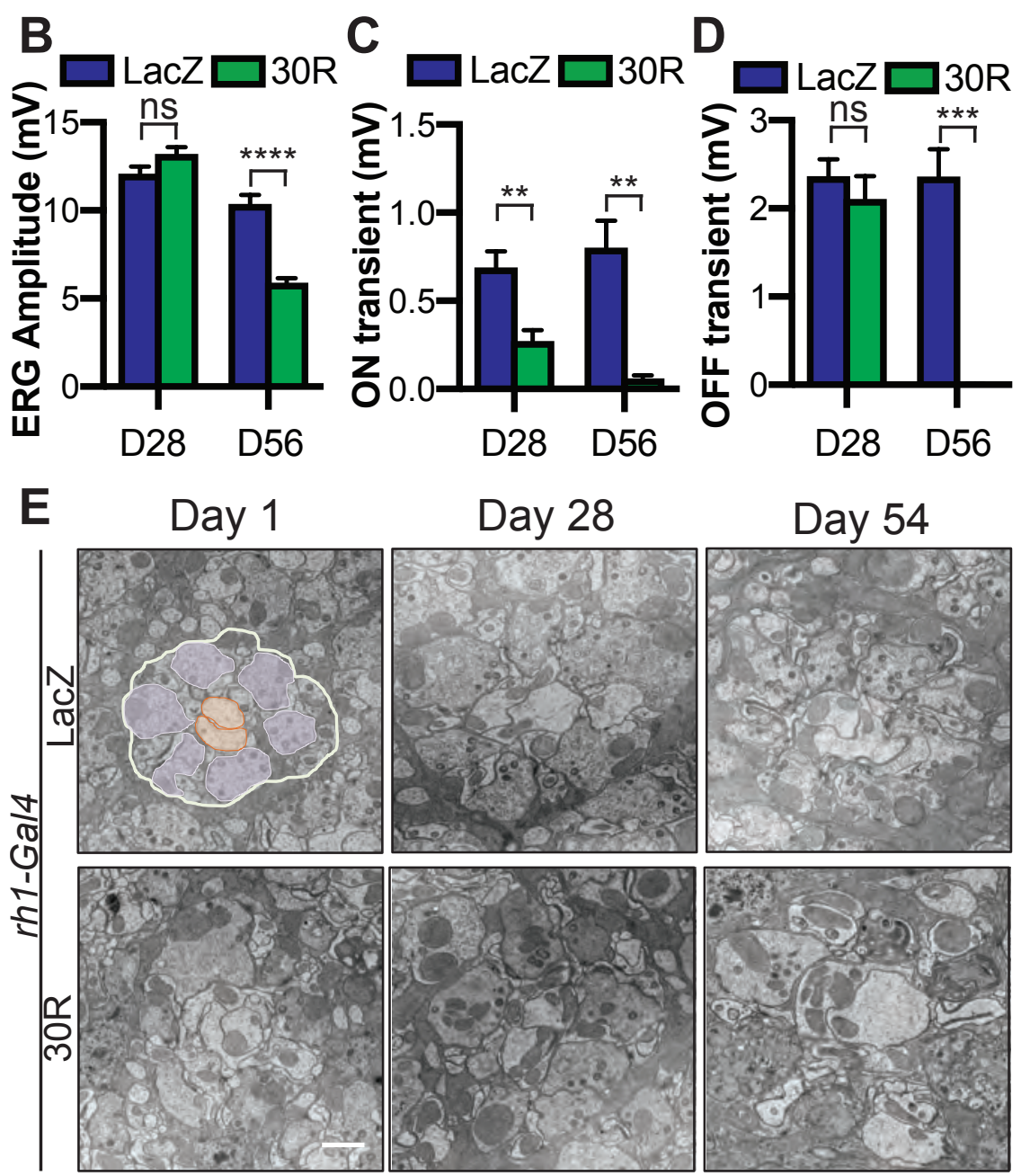

Day 54

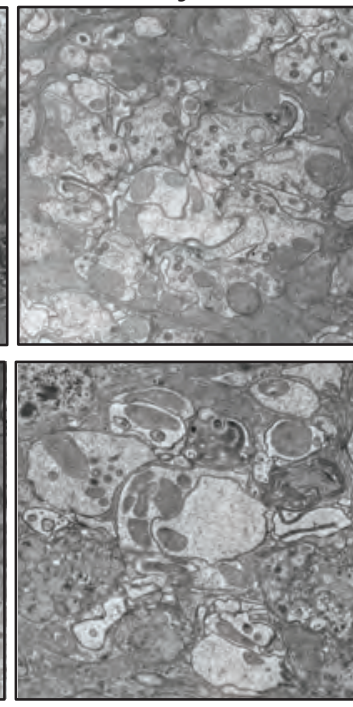

Supplemental Figure 3 Cunningham et al. 


\section{Supplemental Figure 3 (Related to Figure 3): Progressive synapse degeneration} in G4C2-expressing photoreceptor neurons. A) Representative electroretinogram (ERG) of rhodopsin1-Gal4 driving LacZ (control) or 30R in adult eyes at Day 28 or Day 56 after eclosion. B) Quantification of mean ERG amplitude in A. C) Quantification of mean ON transient amplitude in A. D) Quantification of mean OFF transient amplitude in A. Student's t-test, $n=10,10,6,7$. E) Transmission electron micrscopy (TEM) of terminals (synapses) in Rhodopsin1-Gal4 driving UAS-LacZ (control) or 30R adult eyes at Day 1, Day 28, and Day 54 after eclosion. For the Day 1 control image, presynaptic terminals are pseudocolored in purple and post-synaptic terminals are pseudocolored in orange. Data represented are mean \pm SEM. Scale bar represents $1 \mu$ m. n.s., not significant ${ }^{*}, p<0.05,{ }^{* *}, p<0.01,{ }^{* * *} p<0.001,{ }^{* * *} p<0.0001$ 

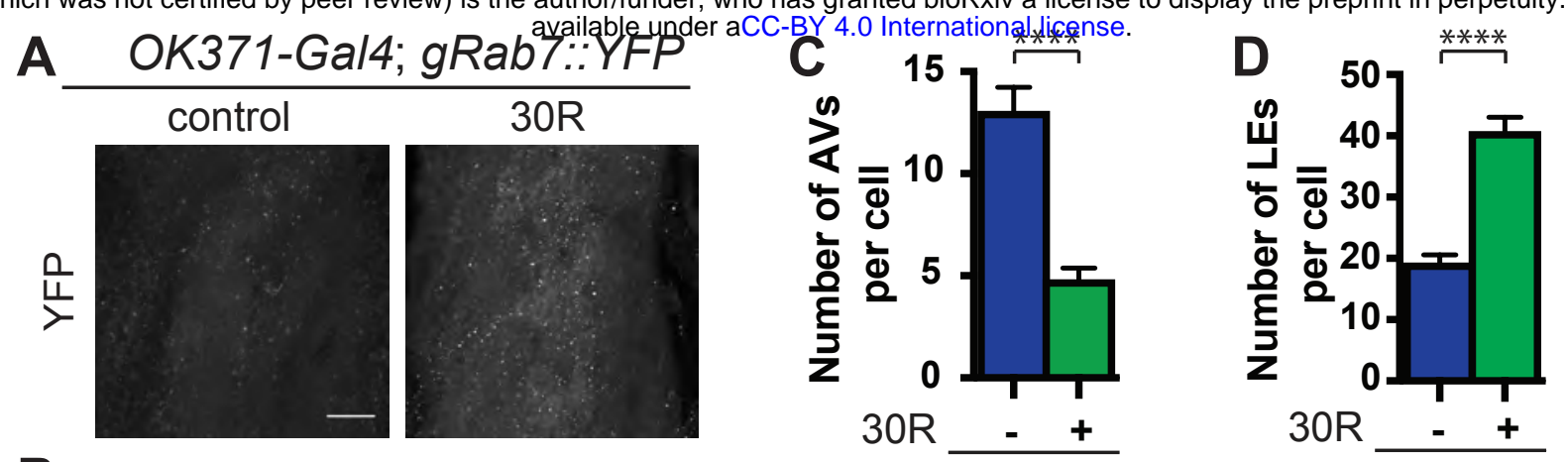

B OK371>Rab7::GFP; mCherry::Atg8

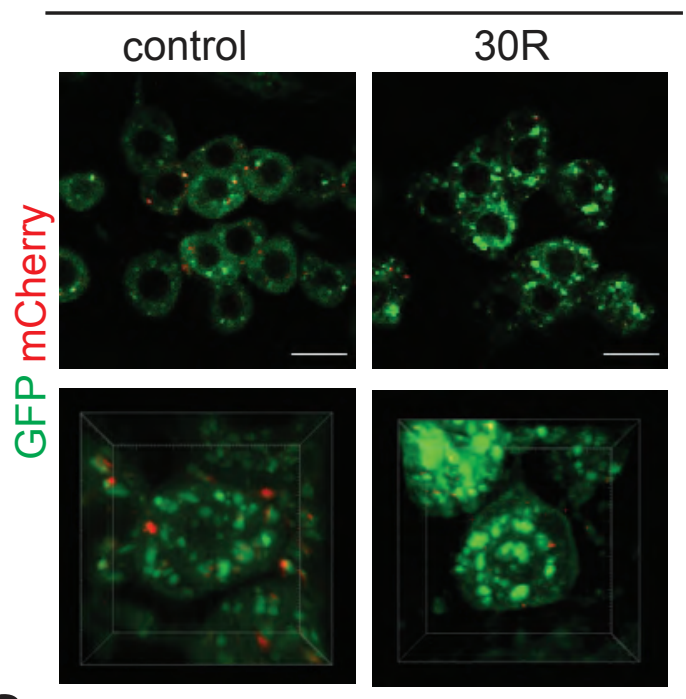

OK371>Rab7::GFP; OK371>Rab7::GFP; mCherry::Atg8 mCherry::Atg8

E

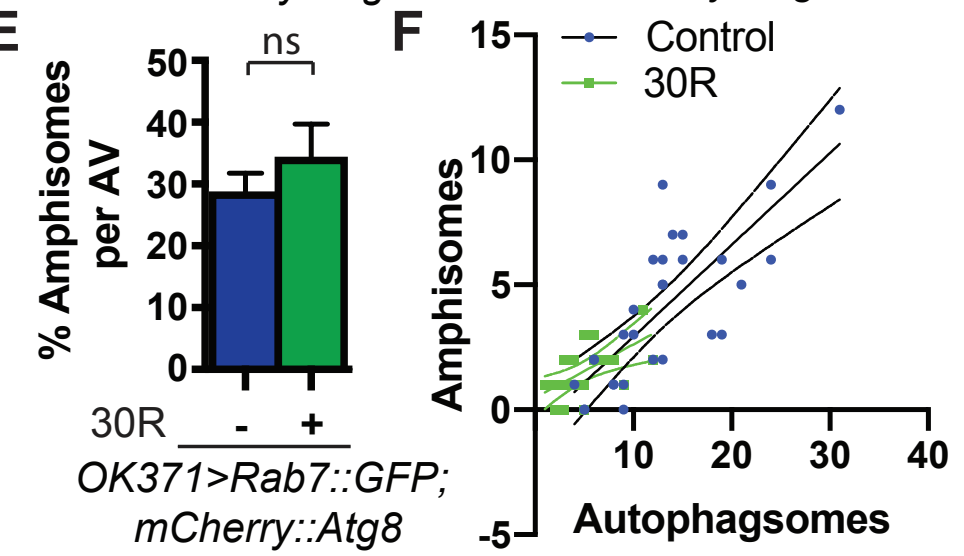

G Rapamycin (uM)

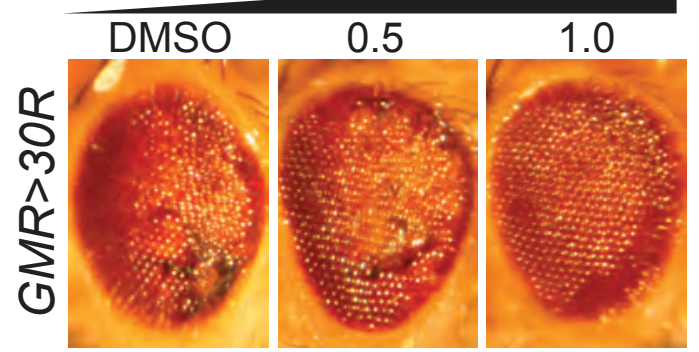

\section{Trehalose (\%)}
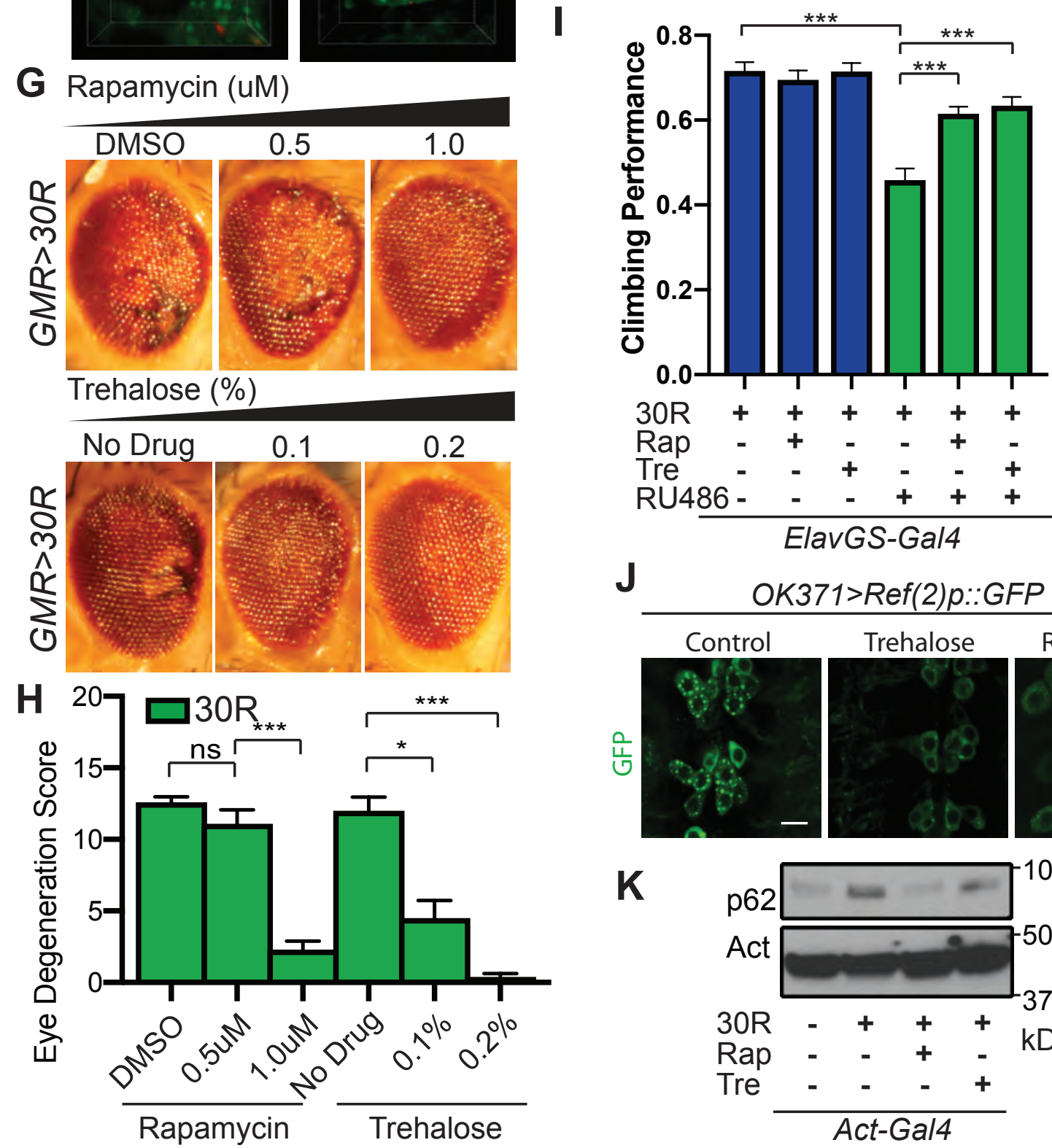

J
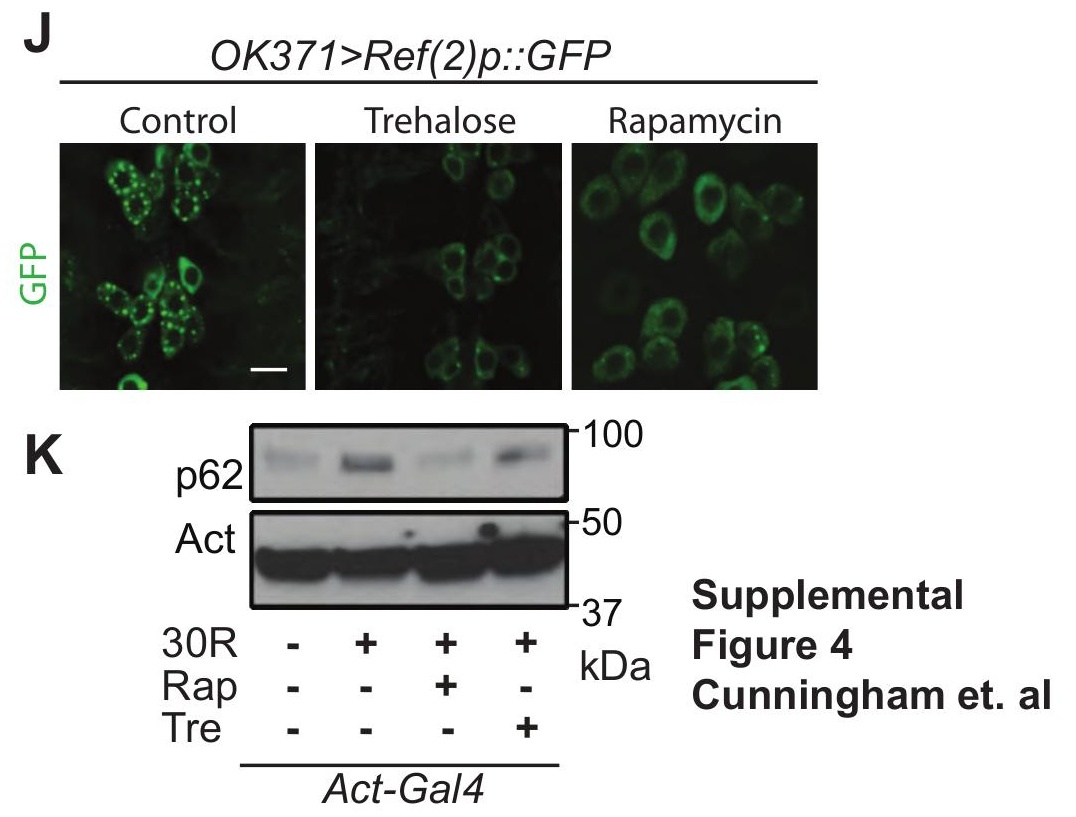


\section{Supplemental Figure 4 (Related to Figure 2 and 3): Rescuing G4C2-mediated}

lysosome defects reduces neurodegeneration. A) $O K 371 /+$ or $O K 371>30 \mathrm{R}$ with genomically tagged Rab7::YFP in the larval ventral nerve cord. B) Control OK371 motor neurons co-expressing UAS-Rab7::GFP and UAS-mCherry::Atg8 in the ventral nerve cord (top). Representative single cell (bottom). C) Quantification of number of Atg8+ vesicles autophagic vesicles per cell, Student's t-test. D) Quantification of number of Rab7+ late endo/lysosomes (LE) per cell (Student's t-test). E) Mean number of amphisomes (co-localized mCherry::Atg8 and Rab7::GFP) per cell normalized to the total number of autophagosomes. F) Number of amphisomes (co-localized mCherry::Atg8 and Rab7::GFP) spots plotted against number of autophagic vesicles for OK371/+ control and OK371>30R motor neurons. G) 15-day old Drosophila eyes expressing 30R using GMR-Gal4 of flies fed with increasing concentrations of rapamycin (DMSO, $0.5 \mu \mathrm{M}$, or $1 \mu \mathrm{M}$ ) and trehalose (no drug, $0.1 \%$, or $0.2 \%$ ). H) Quantification of degeneration in (G), One-way ANOVA, followed by multiple comparisons. I) Adult flies expressing 30R under the control of the inducible, panneuronal elavGS-Gal4 driver have decreased climbing ability after 7 days compared to non-induced controls, which is rescued by supplementing the food with rapamycin or trehalose, One-way ANOVA with Bonferroni's Multiple Comparison's test. (J) Drosophila motor neurons expressing Ref(2)p::GFP and 30R from $3^{\text {rd }}$ instar larvae fed DMSO (control) or $1.0 \mu \mathrm{M}$ rapamycin or $0.2 \%$ trehalose. (E) Western of whole Drosophila larvae expressing no repeats or $30 \mathrm{R}$ and fed DMSO or $1.0 \mathrm{MM}$ rapamycin or $0.2 \%$ trehalose and blotted for $\operatorname{Ref}(2) p$. Data represented are mean + SEM. Scale bars represent $10 \mu$ m. n.s., not significant ${ }^{*}, p<0.05,{ }^{* * *}, p<0.001,{ }^{* * *}, p<0.0001$ 
bioRxiv preprint doi: https://doi.org/10.1101/2020.06.26.173021; this version posted June 26, 2020. The copyright holder for this preprint (which was not certified by peer review) is the author/funder, who has granted bioRxiv a license to display the preprint in perpetuity. It is made A available under aCC-BY 4.0 International license.

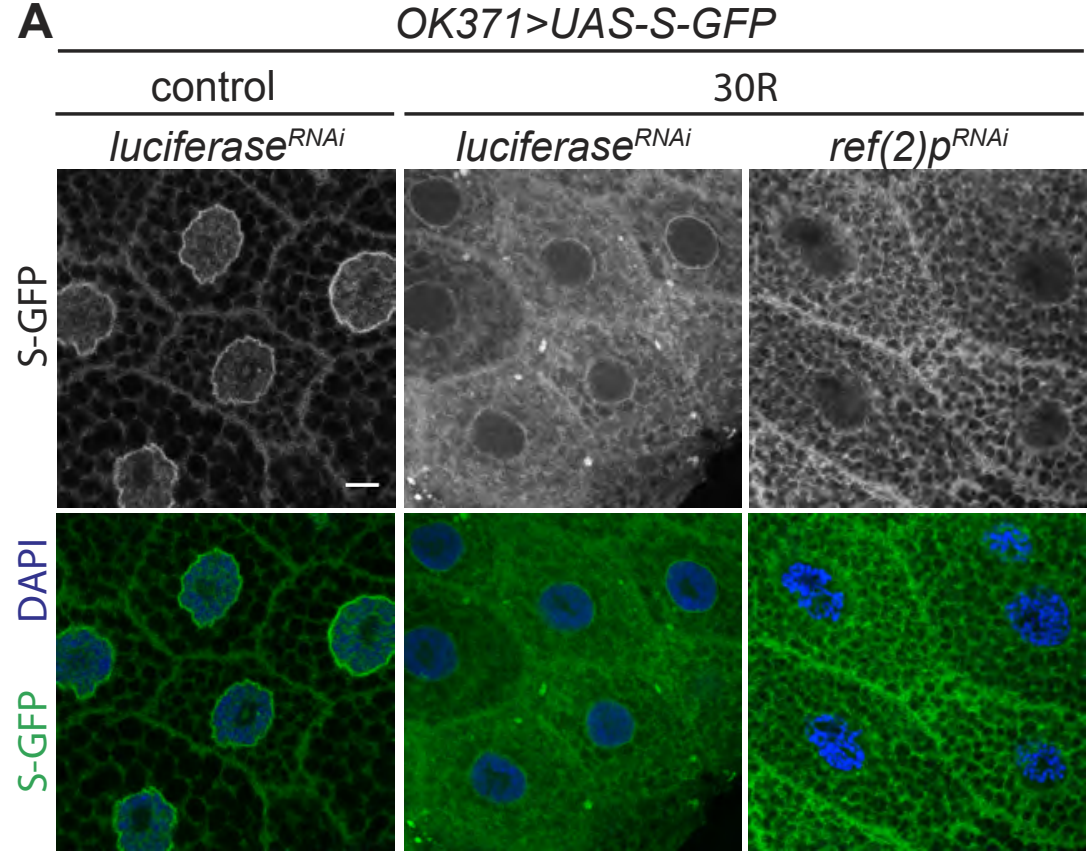

B

OK371>UAS-S-GFP; 30R

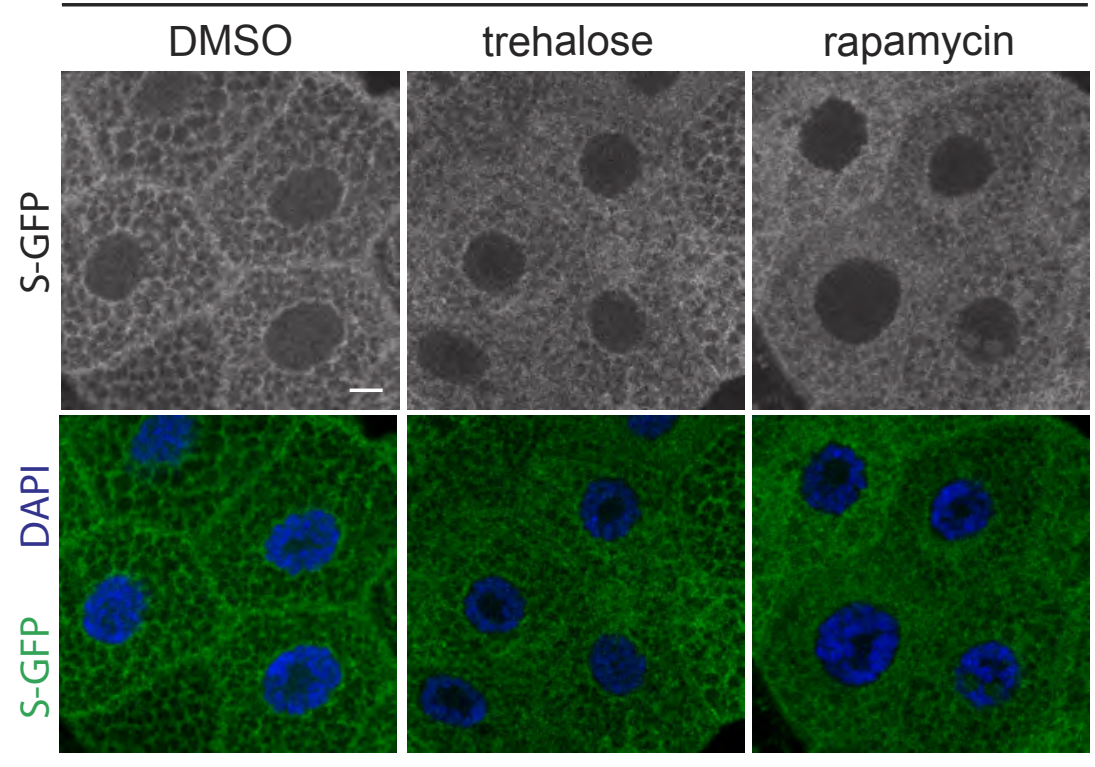

C

OK371>p62::GFP
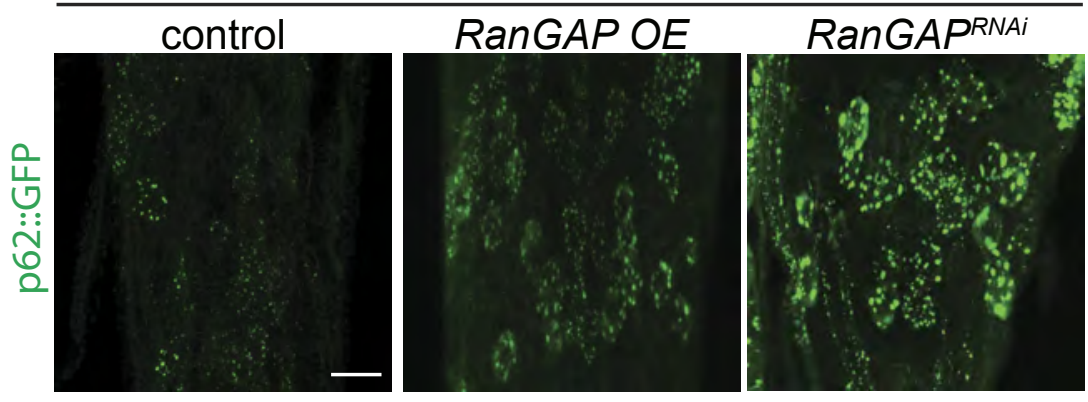

Supplemental Figure 5

Cunningham et al 


\section{Supplemental Figure 5 (Related to Figure 4). Nucleocytoplasmic transport}

disruption is upstream of autophagic defects. (A) Images of the nucleocytoplasmic transport marker shuttle-GFP (S-GFP) in control and OK371>30R with either luciferase (control) or $\operatorname{Ref}(2) p$ RNAi expressed in Drosophila salivary glands. (B) Images of L3 salivary gland expressing S-GFP in OK371>30R Drosophila after feeding supplemented with either control (DMSO), $0.2 \%$ trehalose, or $1.0 \mu \mathrm{m}$ rapamycin. C) Representative images of ventral nerve cords expressing either control, knockdown of RanGAP (UASRanGAPRAi), of overexpression of RanGAP (UAS-RanGAP), a master regulator of nucleocytoplasmic transport along with autophagy receptor Ref(2)P::GFP in OK371 motor neurons. Data represented are mean \pm SEM. Scale bars represent $10 \mu \mathrm{m}$. 


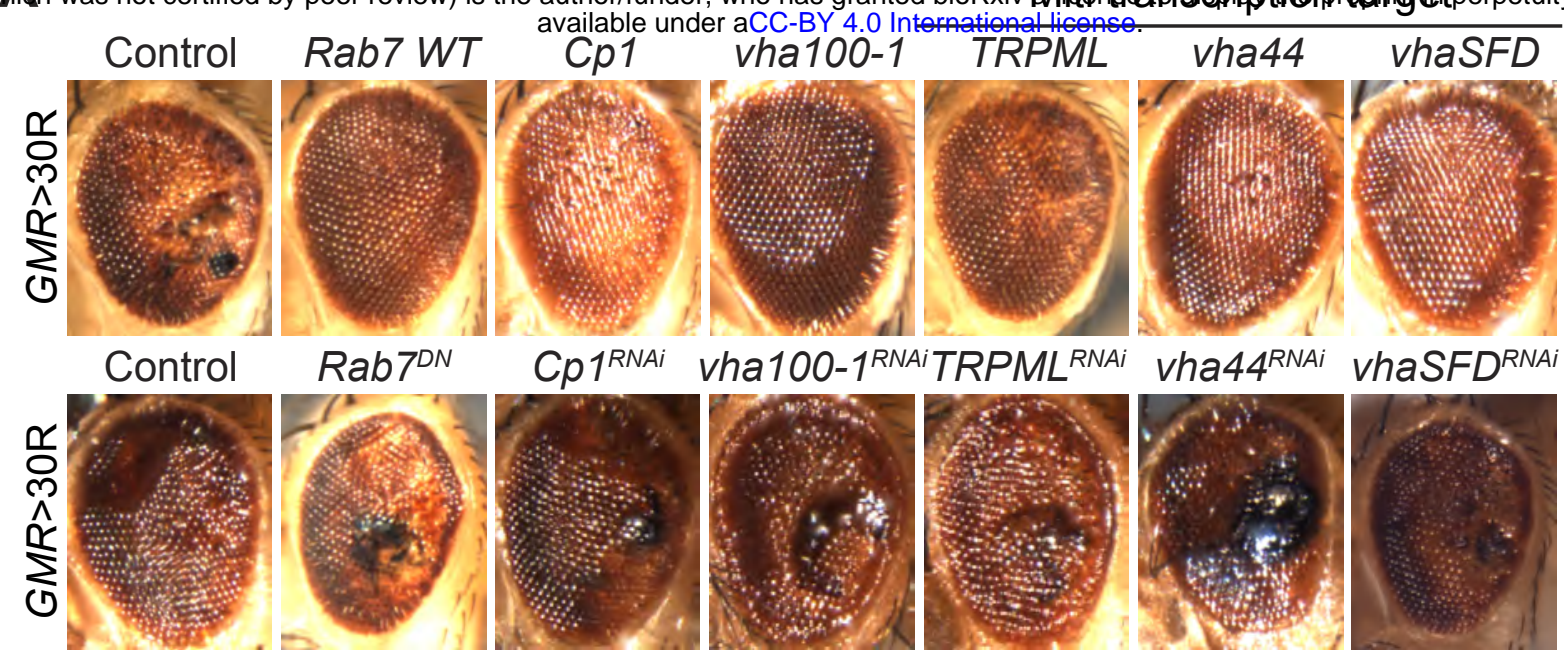

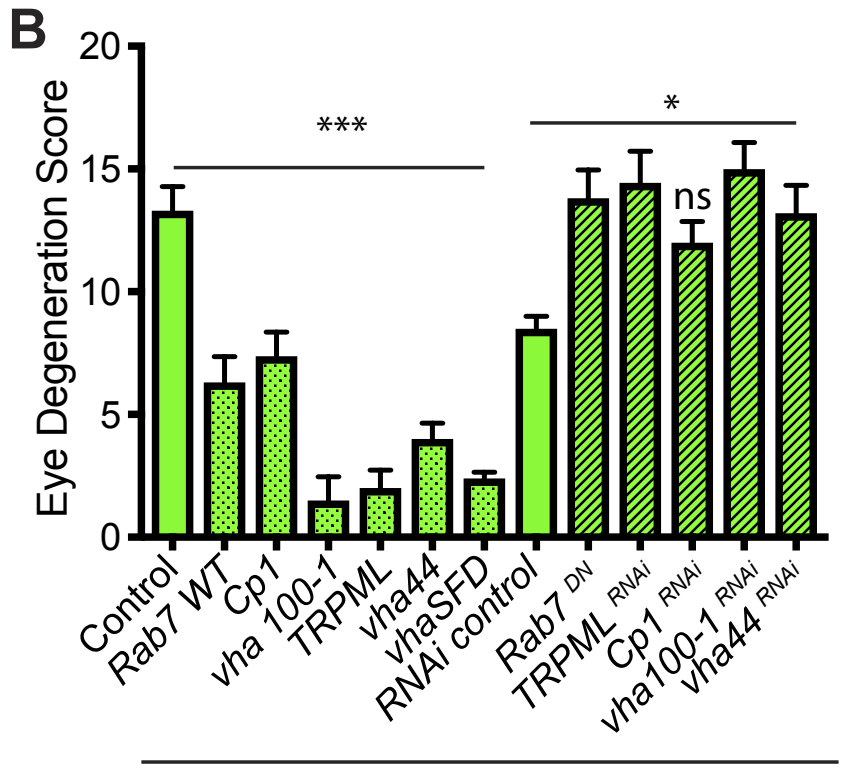

$G M R>30 R$
C

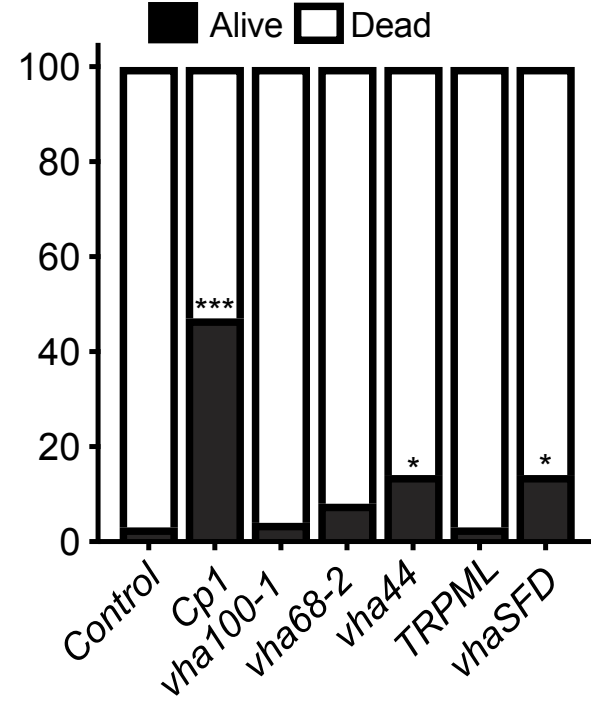

OK371>30R

\section{Supplemental Figure 6 (Related to Figure 6). Genetic manipulation of lysosomes}

rescues degeneration caused by G4C2 expression. A) 15-day old Drosophila eyes

expressing 30R under the control of the GMR-Gal4 driver accompanying

overexpression (top) or knockdown (bottom) of lysosomal genes. (B) Quantification of external eye degeneration in (A), see methods. One-way ANOVA, ${ }^{*}, p<0.05,{ }^{* * *}, p<0.001$ $\mathrm{N}=10,10,10,10,8,4,5,8$ adult flies. (C) Percent of pupal eclosion in Drosophila expressing 30R under the control of the motor neuron OK371-Gal4 driver with UAS-lacZ (control), or overexpression of lysosomal genes $\mathrm{N}=89,120,88,146,119,123,105$ pupa from 3 replicates, Fisher's exact test, ${ }^{*}, \mathrm{p}<.0 .05,{ }^{* * *}, \mathrm{p}<0.001$ Data represented are mean + SEM 

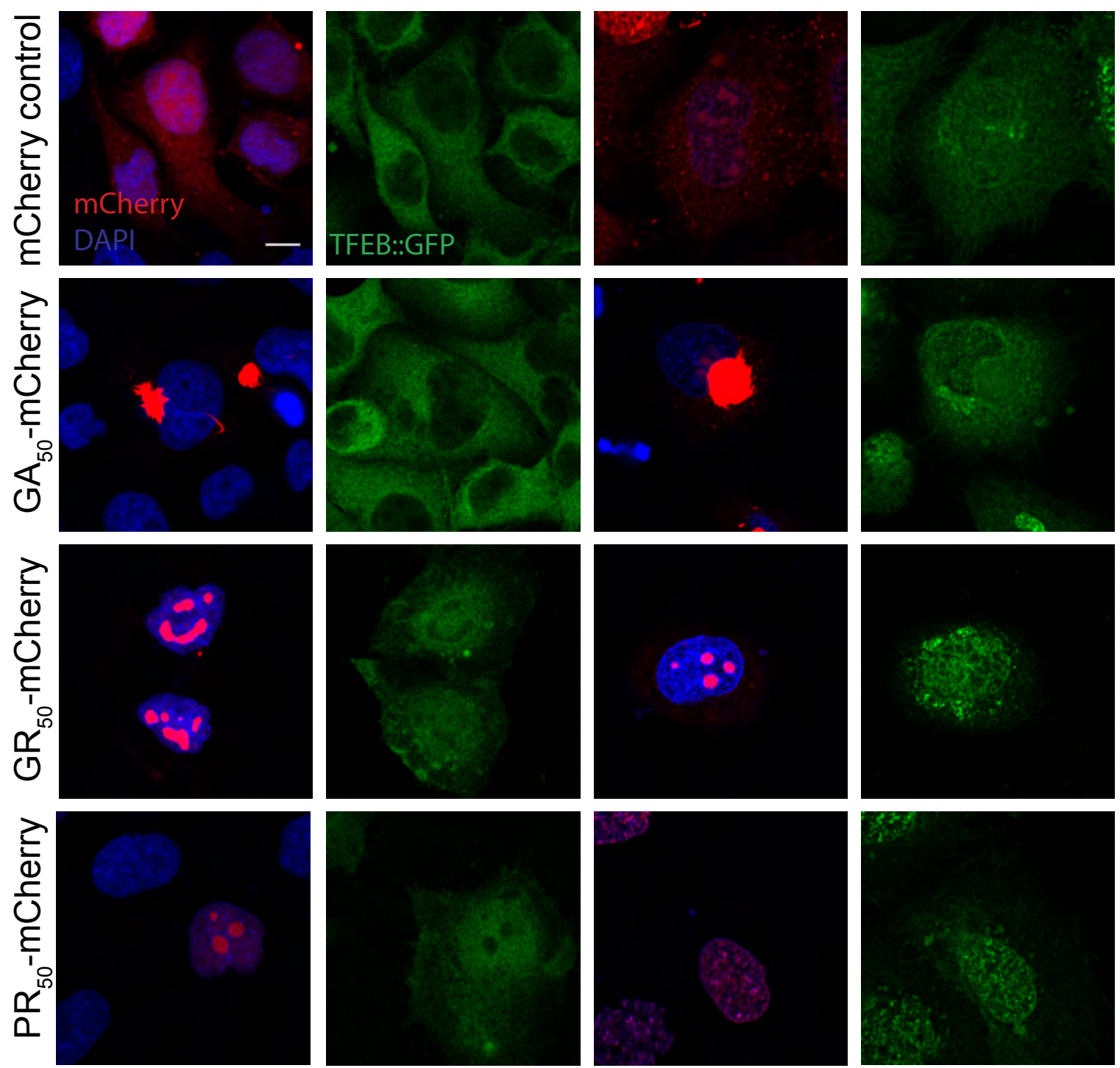

B

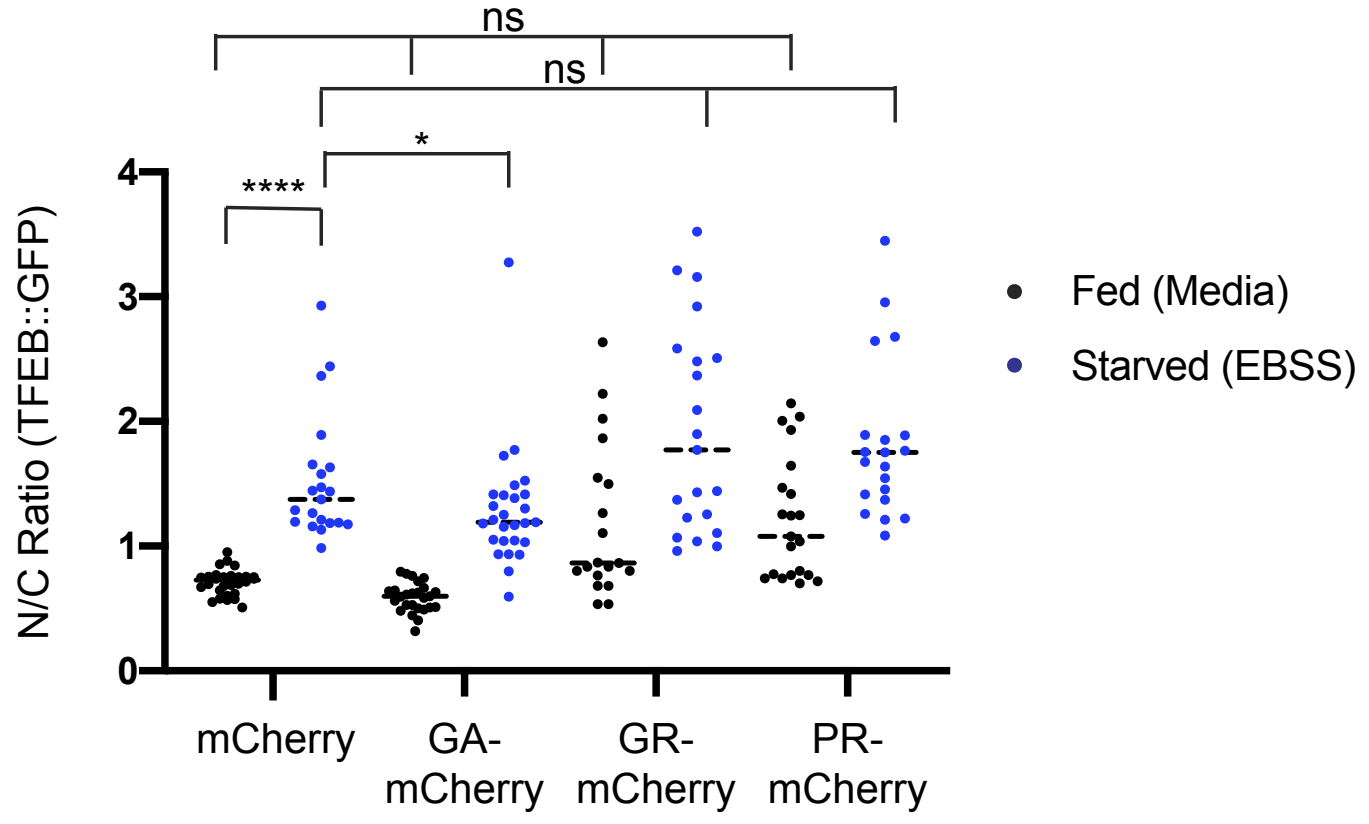

C

Nuclear Fraction

Cytoplasmic Fraction
C9-ALS: actin

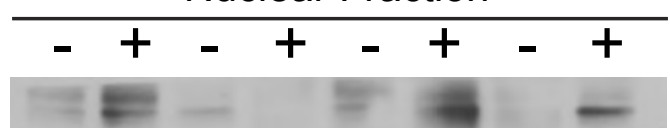

histone $\mathrm{H} 3$

$\begin{array}{lllllll}- & + & - & - & + & - & +\end{array}$

\section{3}




\section{Supplemental Figure 7 (Related to Figure 7). DPRs affect TFEB import in HeLa}

Cells. A) Representative images of transfection of codon-optimized poly-GA-mCherry, poly-GR-mCherry, and poly-PR-mCherry into HeLa cells with stably incorporated TFEBGFP. B) Quantification of (A), One-way ANOVA with multiple comparisons. C) Nuclear and cytoplasmic fractions of human motor cortex samples in Figure $7 \mathrm{c}$ blotted for cytoplasmic (actin) and nuclear (histone H3) housekeeping controls. Scale bars represent 20um. n.s., not significant ${ }^{*}, p<0.05,{ }^{* *}, p<0.01,{ }^{* * *} p<0.001,{ }^{* * * *} p<0.0001$ 


\section{Supplemental Tables}

Supplemental Table 1. Candidate Screen of autophagy-related genes. Flies expressing 30 G4C2 repeats in the eye under control of GMR-GAL4 were crossed to the indicated UAS

5 line and scored for enhancement $(<0)$ or suppression $(>0)$ as described (Zhang et al., 2015a).

\begin{tabular}{|c|c|c|}
\hline Drosophila stock & Effect on gene & Eye Score \\
\hline UAS-Atg6rNAi & RNAi line & -3.5 \\
\hline UAS-Atg18aRNAi & RNAi line & -3 \\
\hline UAS-Atg 1 & cDNA overexpression & -3 \\
\hline UAS-Atg7 & cDNA overexpression & -2.5 \\
\hline UAS-Atg101 RNAi & RNAi line & -2 \\
\hline UAS-Atg8aRNAi & RNAi line & -2 \\
\hline UAS-Atg5RNAi & RNAi line & -2 \\
\hline UAS-Atg6RNAi & RNAi line & -2 \\
\hline UAS-Atg8aRNAi & RNAi line & -2 \\
\hline UAS-Ref(2)p prNAi & RNAi line & -2 \\
\hline UAS-Atg14 RNAi & RNAi line & -2 \\
\hline UAS-Atg16RNAi & RNAi line & -1.5 \\
\hline UAS-Atg16 $6_{R N A i}$ & RNAi line & -1.5 \\
\hline UAS-Atg17 $7_{R N A i}$ & RNAi line & -1.5 \\
\hline UAS-Atg6rNAi & RNAi line & -1 \\
\hline UAS-Atg8bRNAi & RNAi line & -1 \\
\hline UAS-bchsRNAi & RNAi line & -1 \\
\hline UAS-Atg8bRNAi & RNAi line & -1 \\
\hline UAS-Atg $9_{R N A i}$ & RNAi line & -0.5 \\
\hline UAS-Atg18aRNAi & RNAi line & -0.5 \\
\hline UAS-GyffRAi & RNAi line & -0.5 \\
\hline Atg600096 & P-element insertion & 0 \\
\hline UAS-Atg4brNAi & RNAi line & 0 \\
\hline$U A S-A \operatorname{tg} 17_{E P}$ & EP overexpression & 0 \\
\hline bchs58 & EMS mutagenesis & 0 \\
\hline UAS-Atg4aRNAi & RNAi line & 0 \\
\hline UAS-Atg4aRNAi & RNAi line & 0 \\
\hline UAS-Atg10 $10_{R N A i}$ & RNAi line & 0 \\
\hline UAS-Atg16 RNAi & RNAi line & 0 \\
\hline UAS-Atg9 $9_{R N A i}$ & RNAi line & 0 \\
\hline UAS-IttRNAi & RNAi line & 0 \\
\hline UAS-Atg $7_{R N A i}$ & RNAi line & 0 \\
\hline Atg7d06996 & P-element insertion & 0 \\
\hline UAS-Atg4aRNAi & RNAi line & 1 \\
\hline UAS-Atg8aRNAi & RNAi line & 1 \\
\hline Bchs 17 & EMS mutagenesis & 1 \\
\hline UAS-Atg8aEP & EP overexpression & 1 \\
\hline UAS-Atg2EP & EP overexpression & 1 \\
\hline UAS-Atg $7_{R N A i}$ & RNAi line & 1 \\
\hline UAS-Atg13RNAi & RNAi line & 1.5 \\
\hline UAS-Atg14RNAi & RNAi line & 1.5 \\
\hline UAS-Atg3EP & EP overexpression & 2 \\
\hline UAS-Atg18bRNAi & RNAi line & 2 \\
\hline
\end{tabular}


bioRxiv preprint doi: $h t t p s: / / d o i . o r g / 10.1101 / 2020.06 .26 .173021$ : this version posted June 26, 2020. The copyright holder for this preprint (which was not certified by peer review) is the author/funder, who has granted bioRxiv a license to display the preprint in perpetuity. It is made available under aCC-BY 4.0 International license.

\begin{tabular}{|l|l|l|}
\hline$U A S-A \operatorname{tg} 2 R N A i$ & RNAi line & 2 \\
\hline Snap29 B6-21 & EMS & 2 \\
\hline UAS-Atg3RNAi & RNAi line & 3 \\
\hline UAS-Atg2RNAi & RNAi line & 3 \\
\hline UAS-Atg18bRNAi & RNAi line & 3 \\
\hline Atg4bPog97 & P-element insertion & 4 \\
\hline UAS-bchs-HA & cDNA overexpression & 4 \\
\hline
\end{tabular}

\section{Supplemental Table 2 (Related to Figure 7): Demographics of human patients}

\begin{tabular}{|l|l|l|l|l|l|l|}
\hline & Patient ID & Diagnosis & Gender & $\begin{array}{l}\text { Age at } \\
\text { death }\end{array}$ & PMI (hrs) & $\begin{array}{l}\text { C9orf72 } \\
\text { mutation }\end{array}$ \\
\hline Control & 70 & $\begin{array}{l}\text { Multiple } \\
\text { Medical }\end{array}$ & Female & 59 & 7 & No \\
\hline & 91181 & Cardiac arrest & Male & 53 & 12 & No \\
\hline & 91268 & Cardiac failure & Male & 76 & 12 & No \\
\hline & 73 & $\begin{array}{l}\text { Cardiovascular } \\
\text { failure }\end{array}$ & Male & 74 & 4 & No \\
\hline C9-ALS & 88 & FTD/ALS & Male & 59 & 10 & Yes \\
\hline & 67 & Familial ALS & Male & 47 & 5 & Yes \\
\hline & 1629 & FTD/ALS & Female & 55 & NA & Yes \\
\hline & Varelli & ALS & Male & 47 & NA & Yes \\
\hline
\end{tabular}

\section{Supplemental Table 3. Drosophila Stocks used in this study}

\begin{tabular}{|c|c|c|c|}
\hline & \multicolumn{3}{|l|}{ Experimental Models: Organisms/Strains } \\
\hline Stock Name & Drosophila Stock & Source & Identifier \\
\hline GMR-Gal4 & $\begin{array}{l}w[1118] ; \\
P\{w[+m W . h s]=G a w B\} \text { VGlut[OK371] }\end{array}$ & BDSC & BDSC:1104 \\
\hline $30 \mathrm{R}$ & $w[1118] ; U A S-\left(G_{4} C_{2}\right)_{30}$ & $\begin{array}{l}\text { Peng Jin (Xu et } \\
\text { al. 2013) }\end{array}$ & $\begin{array}{l}\text { FlyBase: } \\
\text { FBal0294759 }\end{array}$ \\
\hline $\begin{array}{l}\text { TRiP } \\
\text { background } \\
\text { control }\end{array}$ & $y[1] v[1] ; P\{y[+t 7.7]=$ CaryP $\} a t t P 2$ & BDSC & BDSC: 36303 \\
\hline $\begin{array}{l}\text { UAS- } \\
\text { Ref(2)pRNAi } 1\end{array}$ & $\begin{array}{l}y[1] s c\left[^{*}\right] v[1] \operatorname{sev}[21] ; P\{y[+t 7.7] \\
v[+t 1.8]=T R i P . H M S 00551\} \text { attP2 }\end{array}$ & BDSC & BDSC: 36111 \\
\hline $\begin{array}{l}\text { UAS-Ref(2)p- } \\
\text { HA }\end{array}$ & & $\begin{array}{l}\text { L.M. Martins (de } \\
\text { Castro et al. } \\
\text { 2013) }\end{array}$ & $\begin{array}{l}\text { Flybase: } \\
\text { FBtp0089618 }\end{array}$ \\
\hline $\begin{array}{l}\text { vGlut-OK371- } \\
\text { Gal4; }\end{array}$ & $\begin{array}{l}w[1118] ; \\
P\{w[+m W . h s]=G a w B\} \text { VGlut[OK371] }\end{array}$ & BDSC & $\begin{array}{l}\text { Flybase: } \\
\text { FBal0194519 }\end{array}$ \\
\hline $\begin{array}{l}\text { UAS- } \\
\text { Ref(2)pRNAi 2; }\end{array}$ & $\begin{array}{l}y[1] s c\left[^{*}\right] v[1] \operatorname{sev}[21] ; P\{y[+t 7.7] \\
v[+t 1.8]=T R i P . H M S 00938\} a t t P 2\end{array}$ & BDSC & BDSC: 33978 \\
\hline elavGS-Gal4 & $w\left[^{\star}\right] ;$ P $\{$ elav-Switch.O\}GSG301 & Adrian Isaacs & $\begin{array}{l}\text { Flybase: } \\
\text { FBtp0015149 }\end{array}$ \\
\hline
\end{tabular}


bioRxiv preprint doi: $h t t p s: / / d o i . o r g / 10.1101 / 2020.06 .26 .173021$; this version posted June 26, 2020. The copyright holder for this preprint (which was not certified by peer review) is the author/funder, who has granted bioRxiv a license to display the preprint in perpetuity. It is made available under aCC-BY 4.0 International license.

\begin{tabular}{|c|c|c|c|}
\hline $\operatorname{Ref}(2) p_{o d 3}$ & & $\begin{array}{l}\text { Eric Baehrecke } \\
\text { (Shravage et al. } \\
\text { 2013) }\end{array}$ & $\begin{array}{l}\text { Flybase: } \\
\text { FBal0032568 }\end{array}$ \\
\hline$U A S-G R_{36}$ & $\begin{array}{l}w[1118] ; P\{\{y[+t 7.7] w[+m C]=U A S- \\
\text { poly-GR.PO-36\}attP40 }\end{array}$ & $\begin{array}{l}\text { Adrian lsaacs } \\
\text { (Mizielinska et al. } \\
\text { 2014) }\end{array}$ & \\
\hline Act-Gal4 & $\begin{array}{l}y[1] w\left[^{*}\right] ; P\{A c t 5 C- \\
\text { GAL4\}17bFO1/TM6B, Tb1 }\end{array}$ & BDSC & $\begin{array}{l}\text { Flybase: } \\
\text { FBti0183703 }\end{array}$ \\
\hline $\begin{array}{l}\text { UAS-Ref(2)p- } \\
\text { GFP }\end{array}$ & & $\begin{array}{l}\text { Thomas Neufeld } \\
\text { (Chang et al } \\
\text { 2009) }\end{array}$ & $\begin{array}{l}\text { Flybase: } \\
\text { FBtp0041098 }\end{array}$ \\
\hline $\begin{array}{l}\text { UAS-mCherry- } \\
\text { Atg8 }\end{array}$ & $\begin{array}{l}y[1] w[1118] ; P\{w[+m C]=U A S p-G F P- \\
m C h e r r y-\text { Atg } 8 a\} 2\end{array}$ & BDSC & BDSC: 37749 \\
\hline $\begin{array}{l}\text { UAS-GFP- } \\
\text { Lamp }\end{array}$ & $\left.w{ }^{*}\right] ; P\{w[+m C]=U A S-G F P-L A M P\} 2$ & $\begin{array}{l}\text { Helmut Kramer } \\
\text { (Pulipparacharuvil } \\
\text { et al. 2005) }\end{array}$ & $\begin{array}{l}\text { Flybase: } \\
\text { FBtp0041063 }\end{array}$ \\
\hline $\begin{array}{l}\text { UAS-3R; } \\
\text { UAS- }\left(G_{4} C_{2}\right)_{3}\end{array}$ & & $\begin{array}{l}\text { Adrian Isaacs } \\
\text { (Mizielinska et al. } \\
\text { 2014) }\end{array}$ & $\begin{array}{l}\text { Flybase: } \\
\text { FBal0304407 }\end{array}$ \\
\hline $\begin{array}{l}\text { UAS-36R; } \\
\text { UAS- }\left(G_{4} C_{2}\right)_{36}\end{array}$ & & $\begin{array}{l}\text { Adrian Isaacs } \\
\text { (Mizielinska et al. } \\
\text { 2014) }\end{array}$ & $\begin{array}{l}\text { Flybase: } \\
\text { FBti0166223 }\end{array}$ \\
\hline UAS-lacZ & $\begin{array}{l}w[1118] ; P\{w[+m C]=U A S- \\
\text { lacZ.NZ\}J312 }\end{array}$ & BDSC & BDSC: 3956 \\
\hline Rh1-Gal4 & $P\{r y[+t 7.2]=r h 1-G A L 4\} 3, r y[506]$ & BDSC & BDSC: 8961 \\
\hline gRab7-YFP & $w[1118] ; T I\{T I\} R a b 7[E Y F P]$ & BDSC & BDSC: 62545 \\
\hline $\begin{array}{l}\text { UAS-Rab7- } \\
\text { GFP }\end{array}$ & & BDSC & BDSC: 42706 \\
\hline $\begin{array}{l}\text { UAS- } \\
\text { luciferasernAi }\end{array}$ & $\begin{array}{l}y[1] v[1] ; P\{y[+t 7.7] \\
v[+t 1.8]=T R i P . J F 01355\} a t t P 2\end{array}$ & BDSC & BDSC: 31603 \\
\hline UAS-S-GFP; & $\begin{array}{l}w[1118] ; P\{w[+m C]=U A S-N L S- \\
N E S[+]-G F P\} 5 A\end{array}$ & BDSC & BDSC: 7032 \\
\hline UAS-RanGAP & & $\begin{array}{l}\text { Lloyd lab (Zhang } \\
\text { et. al 2015) }\end{array}$ & \\
\hline $\begin{array}{l}\text { UAS- } \\
\text { RanGAPRNAi; }\end{array}$ & $\begin{array}{l}y[1] v[1] ; P\{y[+t 7.7] \\
v[+t 1.8]=T R i P . J F 03244\} a t t P 2 / T M 3, \\
S b[1]\end{array}$ & BDSC & BDSC: 29565 \\
\hline $\begin{array}{l}\text { UAS-CD8- } \\
\text { GFP }\end{array}$ & $\begin{array}{l}y[1] w\left[{ }^{*}\right] ; P\{w[+m C]=U A S- \\
m C D 8:: G F P . L\} L L 5\end{array}$ & BDSC & $\begin{array}{l}\text { Flybase: } \\
\text { FBti0012685 }\end{array}$ \\
\hline UAS-Mitf-HA & & $\begin{array}{l}\text { Francesca } \\
\text { Pignoni (Zhang et } \\
\text { al. 2015) }\end{array}$ & \\
\hline daGS-Gal4 & $w\left[^{\star}\right] ; P\{w[+m C]=d a-G S G A L 4 . T\}$ & & $\begin{array}{l}\text { Flybase: } \\
\text { FBtp0057039 }\end{array}$ \\
\hline $\begin{array}{l}\text { UAS- } \\
\text { embargoed }\end{array}$ & $\begin{array}{l}y[1] v[1] ; P\{y[+t 7.7] \\
v[+t 1.8]=T R i P . J F 01311\} a t t P 2\end{array}$ & BDSC & BDSC: 31353 \\
\hline $\begin{array}{l}\text { Mitf } \\
\text { duplication; } \\
\text { MitfSI-RES }\end{array}$ & & $\begin{array}{l}\text { Francesca } \\
\text { Pignoni (Zhang et } \\
\text { al. 2015b) }\end{array}$ & $\begin{array}{l}\text { Flybase: } \\
\text { FBtp0115483 }\end{array}$ \\
\hline Mitf RNAi & $\begin{array}{l}y[1] s c\left[^{*}\right] v[1] \operatorname{sev}[21] ; P\{y[+t 7.7] \\
v[+t 1.8]=\text { TRiP.HMS02712\}attP2 }\end{array}$ & BDSC & BDSC: 43998 \\
\hline
\end{tabular}


bioRxiv preprint doi: https://doi.org/10.1101/2020.06.26.173021: this version posted June 26, 2020. The copyright holder for this preprint (which was not certified by peer review) is the author/funder, who has granted bioRxiv a license to display the preprint in perpetuity. It is made available under aCC-BY 4.0 International license.

\begin{tabular}{|c|c|c|c|}
\hline$U A S-R a b 7 w T$ & $\begin{array}{l}y[1] w[*] ; P\{w[+m C]=U A S p- \\
\text { YFP.Rab7 }\} 21 / S M 5\end{array}$ & BDSC & BDSC: 23641 \\
\hline UAS-CP1EP & $\begin{array}{l}y[1] w[67 c 23] ; P\{w[+m C] \\
y[+m D i n t 2]=E P g y 2\} C p 1[E Y 05806]\end{array}$ & BDSC & BDSC: 15957 \\
\hline $\begin{array}{l}\text { UAS-Vha100- } \\
\text { 1EP }\end{array}$ & $\begin{array}{l}w[1118] ; P\{w[+m C]=E P\} \text { Vha100- } \\
1[G 4514] / T M 6 C, S b[1]\end{array}$ & BDSC & BDSC: 63269 \\
\hline UAS-TRPML & & $\begin{array}{l}\text { Kartik } \\
\text { Venkatachalam }\end{array}$ & $\begin{array}{l}\text { Flybase: } \\
\text { FBti0162438 }\end{array}$ \\
\hline UAS-Vha44EP & $\begin{array}{l}y[1] w[67 c 23] ; P\{w[+m C] \\
y[+m \text { Dint2] }=E P g y 2\} \text { Vha44[EY02202] }\end{array}$ & BDSC & BDSC: 20140 \\
\hline $\begin{array}{l}\text { UAS- } \\
\text { VhaSFDEP }\end{array}$ & $\begin{array}{l}y[1] w[67 c 23] ; P\{w[+m C] \\
y[+m D i n t 2]=E P g y 2\} \text { VhaSFD[EY04644] } \\
/ C y O\end{array}$ & BDSC & BDSC: 15758 \\
\hline$U A S-R a b 7 D N$ & $\begin{array}{l}y[1] w\left[{ }^{\star}\right] ; P\{w[+m C]=U A S p- \\
\text { YFP.Rab7.T22N\}06 }\end{array}$ & BDSC & BDSC: 9778 \\
\hline UAS-CP1RNAi & $\begin{array}{l}y[1] s c\left[^{*}\right] v[1] \operatorname{sev}[21] ; P\{y[+t 7.7] \\
v[+t 1.8]=T R i P . H M S 00725\} a t t P 2\end{array}$ & BDSC & BDSC: 32932 \\
\hline $\begin{array}{l}\text { UAS-Vha100- } \\
1_{\text {RNAi }}\end{array}$ & $\begin{array}{l}y[1] v[1] ; P\{y[+t 7.7] \\
v[+t 1.8]=T R i P . J F 02059\} a t t P 2\end{array}$ & BDSC & BDSC: 26290 \\
\hline $\begin{array}{l}\text { UAS- } \\
\text { TRPMLRNAi }\end{array}$ & $\begin{array}{l}y[1] v[1] ; P\{y[+t 7.7] \\
v[+t 1.8]=T R i P . J F 01239\} a t t P 2\end{array}$ & BDSC & BDSC: 31294 \\
\hline $\begin{array}{l}\text { UAS- } \\
\text { Vha44RNAi }\end{array}$ & $\begin{array}{l}y[1] s c\left[^{*}\right] v[1] \operatorname{sev}[21] ; P\{y[+t 7.7] \\
v[+t 1.8]=T R i P . H M S 00821\} \text { attP2 }\end{array}$ & BDSC & BDSC: 33884 \\
\hline $\begin{array}{l}\text { UAS- } \\
\text { VhaSFD RNAi }\end{array}$ & $\begin{array}{l}y[1] s c\left[{ }^{*}\right] v[1] \operatorname{sev}[21] ; P\{y[+t 7.7] \\
v[+t 1.8]=T R i P . H M S 02144\} \text { attP40 }\end{array}$ & BDSC & BDSC: 40896 \\
\hline UAS-Atg6RNAi & $\begin{array}{l}y[1] s c\left[^{*}\right] v[1] ; P\{y[+t 7.7] \\
v[+t 1.8]=T R i P . H M S 01483\} \text { attP2 }\end{array}$ & BDSC & BDSC: 35741 \\
\hline $\begin{array}{l}\text { UAS- } \\
\text { Atg18aRNAi }\end{array}$ & $\begin{array}{l}\left.y[1] s c{ }^{*}\right] v[1] ; P\{y[+t 7.7] \\
v[+t 1.8]=\text { TRiP.HMS01193\}attP2 }\end{array}$ & BDSC & BDSC: 34714 \\
\hline UAS-Atg1 & $y[1] w\left[^{*}\right] ; P\{w[+m C]=U A S-\operatorname{Atg} 1 . S\} 6 B$ & BDSC & BDSC: 51655 \\
\hline UAS-Atg7 & $w[1118] ; P\{w[+m C]=U A S-A \operatorname{tg} 7\}$ & BDSC & NA \\
\hline $\begin{array}{l}\text { UAS- } \\
\text { Atg101 RNAi }\end{array}$ & $\begin{array}{l}y[1] s c\left[^{*}\right] v[1] ; P\{y[+t 7.7] \\
v[+t 1.8]=T R i P . H M S 01349\} \text { attP2 }\end{array}$ & BDSC & BDSC: 34360 \\
\hline UAS-Atg8aRnAi & $\begin{array}{l}\left.y[1] s c{ }^{\star}\right] v[1] ; P\{y[+t 7.7] \\
v[+t 1.8]=T R i P . H M S 01328\} a t t P 2\end{array}$ & BDSC & BDSC: 34340 \\
\hline UAS-Atg5RNAi & $\begin{array}{l}y[1] v[1] ; P\{y[+t 7.7] \\
v[+t 1.8]=T R i P . J F 02703\} \text { attP2 }\end{array}$ & BDSC & BDSC: 27551 \\
\hline UAS-Atg6 $6_{R N A i}$ & $\begin{array}{l}y[1] s c\left[^{*}\right] v[1] ; P\{y[+t 7.7] \\
v[+t 1.8]=T R i P . H M S 01244\} a t t P 2\end{array}$ & BDSC & BDSC: 34899 \\
\hline UAS-Atg8aRNAi & $\begin{array}{l}y[1] v[1] ; P\{y[+t 7.7] \\
v[+t 1.8]=T R i P . J F 02895\} a t t P 2 \\
e\left[{ }^{*}\right] / T M 3, S b[1]\end{array}$ & BDSC & BDSC: 28989 \\
\hline $\begin{array}{l}\text { UAS- } \\
\operatorname{Ref}(2) p_{R N A i}\end{array}$ & $\begin{array}{l}y[1] s c\left[^{*}\right] v[1] ; P\{y[+t 7.7] \\
v[+t 1.8]=T R i P . H M S 00551\} \text { attP2 }\end{array}$ & BDSC & $\begin{array}{l}\text { BDSC: } 36111 \\
(\operatorname{Ref}(2) p K D 1)\end{array}$ \\
\hline UAS-Atg14RNAi & $\begin{array}{l}y[1] s c\left[^{*}\right] v[1] ; P\{y[+t 7.7] \\
v[+t 1.8]=\text { TRiP.GL00318\}attP2 }\end{array}$ & BDSC & BDSC: 55398 \\
\hline UAS-Atg16RNAi & $\begin{array}{l}y[1] s c\left[^{*}\right] v[1] ; P\{y[+t 7.7] \\
v[+t 1.8]=\text { TRiP.HMS01347\}attP2 }\end{array}$ & BDSC & BDSC: 34358 \\
\hline
\end{tabular}


bioRxiv preprint doi: https://doi.org/10.1101/2020.06.26.173021: this version posted June 26, 2020. The copyright holder for this preprint (which was not certified by peer review) is the author/funder, who has granted bioRxiv a license to display the preprint in perpetuity. It is made available under aCC-BY 4.0 International license.

\begin{tabular}{|c|c|c|c|}
\hline UAS-Atg16RNAi & $\begin{array}{l}y[1] v[1] ; P\{y[+t 7.7] \\
v[+t 1.8]=T R i P . H M J 22265\} a t t P 40 / C y O\end{array}$ & BDSC & BDSC: 58244 \\
\hline$U A S-A \operatorname{tg} 17_{R N A i}$ & $\begin{array}{l}y[1] s c\left[^{*}\right] v[1] ; P\{y[+t 7.7] \\
v[+t 1.8]=T R i P . H M S 01611\} \text { attP2/TM3 } \\
S b[1]\end{array}$ & BDSC & BDSC: 36918 \\
\hline UAS-Atg6RNAi & $\begin{array}{l}y[1] v[1] ; P\{y[+t 7.7] \\
v[+t 1.8]=T R i P . J F 02897\} a t t P 2\end{array}$ & BDSC & BDSC: 28060 \\
\hline UAS-Atg8bRNAi & $\begin{array}{l}y[1] s c\left[^{*}\right] v[1] ; P\{y[+t 7.7] \\
v[+t 1.8]=\text { TRiP.HMS01245\}attP2 }\end{array}$ & BDSC & BDSC: 34900 \\
\hline UAS-bchsRNAi & $\begin{array}{l}y[1] v[1] ; P\{y[+t 7.7] \\
v[+t 1.8]=\text { TRiP.HMJ02083\}attP40 }\end{array}$ & BDSC & BDSC: 42517 \\
\hline UAS-Atg8brNAi & $\begin{array}{l}y[1] v[1] ; P\{y[+t 7.7] \\
v[+t 1.8]=\text { TRiP.JF02706\}attP2 }\end{array}$ & BDSC & BDSC: 27554 \\
\hline$U A S-A t g 9_{R N A i}$ & $\begin{array}{l}y[1] v[1] ; P\{y[+t 7.7] \\
v[+t 1.8]=T R i P . J F 02891\} \text { attP2 }\end{array}$ & BDSC & BDSC: 28055 \\
\hline $\begin{array}{l}\text { UAS- } \\
\text { Atg18aRNAi }\end{array}$ & $\begin{array}{l}y[1] v[1] ; P\{y[+t 7.7] \\
v[+t 1.8]=T R i P . J F 02898\} a t t P 2\end{array}$ & BDSC & BDSC: 28061 \\
\hline UAS-Gyf $f_{R N A i}$ & $\begin{array}{l}y[1] v[1] ; P\{y[+t 7.7] \\
v[+t 1.8]=\text { TRiP.HM05106\}attP2 }\end{array}$ & BDSC & BDSC: 28896 \\
\hline Atg600096 & $\begin{array}{l}r y[506] P\{r y[+t 7.2]=P Z\} A t g 6[00096] / \\
T M 3, \operatorname{ry}[R K] \text { Sb[1] Ser[1] }\end{array}$ & BDSC & BDSC: 11487 \\
\hline UAS-Atg4bRNAi & $\begin{array}{l}y[1] v[1] ; P\{y[+t 7.7] \\
v[+t 1.8]=\text { TRiP.HMS04249\}attP2 }\end{array}$ & BDSC & BDSC: 56046 \\
\hline UAS-Atg17EP & $\begin{array}{l}y[1] w[67 c 23] ; P\{w[+m C] \\
y[+m D \text { int2]=EPgy2 }\} \text { Atg 17[EY03045] }\end{array}$ & BDSC & BDSC: 15618 \\
\hline bchs58 & $\begin{array}{l}y[1] w\left[{ }^{*}\right] ; P\{w[+m C]=E P\} E P 2299, \\
b c h s[58] / C y O\end{array}$ & BDSC & BDSC: 9887 \\
\hline UAS-Atg4aRNAi & $\begin{array}{l}y[1] s c\left[^{\star}\right] v[1] ; P\{y[+t 7.7] \\
v[+t 1.8]=T R i P . H M S 01482\} \text { attP2 }\end{array}$ & BDSC & BDSC: 35740 \\
\hline UAS-Atg4aRNAi & $\begin{array}{l}y[1] v[1] ; P\{y[+t 7.7] \\
v[+t 1.8]=\text { TRiP.GLC01355\}attP40 }\end{array}$ & BDSC & BDSC: 44421 \\
\hline UAS-Atg10RNAi & $\begin{array}{l}y[1] v[1] ; P\{y[+t 7.7] \\
v[+t 1.8]=\text { TRiP.HMS02026\}attP40 }\end{array}$ & BDSC & BDSC: 40859 \\
\hline UAS-Atg16RNAi & $\begin{array}{l}y[1] s c\left[^{*}\right] v[1] ; P\{y[+t 7.7] \\
v[+t 1.8]=\text { TRiP.HMS01347\}attP2 }\end{array}$ & BDSC & BDSC: 34358 \\
\hline$U A S-A \operatorname{tg} 9_{R N A i}$ & $\begin{array}{l}y[1] s c\left[^{*}\right] v[1] ; P\{y[+t 7.7] \\
v[+t 1.8]=\text { TRiP.HMS01246\}attP2 }\end{array}$ & BDSC & BDSC: 34901 \\
\hline UAS-ItRNAi & $\begin{array}{l}\left.y[1] s c{ }^{*}\right] v[1] ; P\{y[+t 7.7] \\
v[+t 1.8]=T R i P . H M S 00190\} \text { attP2/TM3 } \\
S b[1]\end{array}$ & BDSC & BDSC: 34871 \\
\hline$U A S-A \operatorname{tg} 7_{R N A i}$ & $\begin{array}{l}y[1] s c\left[^{\star}\right] v[1] ; P\{y[+t 7.7] \\
v[+t 1.8]=T R i P . H M S 01358\} \text { attP2/TM3, } \\
S b[1]\end{array}$ & BDSC & BDSC: 34369 \\
\hline Atg7do6996 & $\begin{array}{l}w[1118] ; \\
P\{w[+m C]=X P\} \operatorname{Atg} 7[d 06996] / C y O\end{array}$ & BDSC & BDSC: 19257 \\
\hline UAS-Atg4aRNAi & $\begin{array}{l}y[1] v[1] ; P\{y[+t 7.7] \\
v[+t 1.8]=T R i P . J F 03003\} \text { attP2 }\end{array}$ & BDSC & BDSC: 28367 \\
\hline UAS-Atg8aRNAi & $\begin{array}{l}y[1] v[1] ; P\{y[+t 7.7] \\
v[+t 1.8]=T R i P . H M J 22416\} a t t P 40\end{array}$ & BDSC & BDSC: 58309 \\
\hline Bchs17 & $\begin{array}{l}y[1] w\left[^{*}\right] ; P\{w[+m C]=E P\} E P 2299, \\
b c h s[17] / C y O\end{array}$ & BDSC & BDSC: 9888 \\
\hline UAS-Atg8aEP & $w[1118] P\{w[+m C]=E P\}$ Atg8a[EP362] & BDSC & BDSC: 10107 \\
\hline$U A S-A t g 2 E P$ & $\begin{array}{l}w[1118] ; \\
P\{w[+m C]=E P\} A \operatorname{tg} 2[E P 3697] / T M 6 B, \\
T b[1]\end{array}$ & BDSC & BDSC: 17156 \\
\hline
\end{tabular}


bioRxiv preprint doi: https://doi.org/10.1101/2020.06.26.173021. this version posted June 26, 2020. The copyright holder for this preprint (which was not certified by peer review) is the author/funder, who has granted bioRxiv a license to display the preprint in perpetuity. It is made available under aCC-BY 4.0 International license.

\begin{tabular}{|c|c|c|c|}
\hline UAS-Atg7 $7_{R N A i}$ & $\begin{array}{l}y[1] s c\left[^{*}\right] v[1] ; P\{y[+t 7.7] \\
v[+t 1.8]=T R i P . H M S 01358\} \text { attP2/TM3, } \\
\text { Sb[1] }\end{array}$ & BDSC & BDSC: 34369 \\
\hline UAS-Atg13RNAi & $\begin{array}{l}y[1] v[1] ; P\{y[+t 7.7] \\
v[+t 1.8]=T R i P . H M S 02028\} a t t P 40\end{array}$ & BDSC & BDSC: 40861 \\
\hline UAS-Atg14RNAi & $\begin{array}{l}y[1] v[1] ; P\{y[+t 7.7] \\
v[+t 1.8]=T R i P . H M S 02025\} a t t P 40 / C y O\end{array}$ & BDSC & BDSC: 40858 \\
\hline$U A S-A \operatorname{tg} 3 E P$ & $\begin{array}{l}y[1] w[67 c 23] ; P\{w[+m C] \\
y[+m D i n t 2]=E P g y 2\} A \operatorname{tg} 3[E Y 08396]\end{array}$ & BDSC & BDSC: 16429 \\
\hline $\begin{array}{l}\text { UAS- } \\
\text { Atg18bRNAi }\end{array}$ & $\begin{array}{l}y[1] s c\left[^{*}\right] v[1] ; P\{y[+t 7.7] \\
v[+t 1.8]=T R i P . H M S 01194\} a t t P 2\end{array}$ & BDSC & BDSC: 34715 \\
\hline$U A S-A \operatorname{tg} 2 R N A i$ & $\begin{array}{l}y[1] v[1] ; P\{y[+t 7.7] \\
v[+t 1.8]=T R i P . J F 02786\} a t t P 2\end{array}$ & BDSC & BDSC: 27706 \\
\hline Snap29 & $\begin{array}{l}w\left[^{*}\right] ; P\{r y[+t 7.2]=n e o F R T\} 42 D \\
\text { Snap29[B6-21]/CyO, } \\
P\{w[+m C]=G A L 4-t w i . G\} 2.2, \\
P\{w[+m C]=U A S-2 x E G F P\} A H 2.2\end{array}$ & BDSC & BDSC: 56818 \\
\hline UAS-Atg3RNAi & $\begin{array}{l}\left.y[1] s C{ }^{*}\right] v[1] ; P\{y[+t 7.7] \\
v[+t 1.8]=\text { TRiP.HMS01348\}attP2 }\end{array}$ & BDSC & BDSC: 34359 \\
\hline UAS-Atg2RNAi & $\begin{array}{l}y[1] s c\left[{ }^{*}\right] v[1] ; P\{y[+t 7.7] \\
v[+t 1.8]=\text { TRiP.HMS01198\}attP2 }\end{array}$ & BDSC & BDSC: 34719 \\
\hline $\begin{array}{l}\text { UAS- } \\
\text { Atg18bRNAi }\end{array}$ & $\begin{array}{l}y[1] s c\left[^{*}\right] v[1] ; P\{y[+t 7.7] \\
v[+t 1.8]=\text { TRiP.HMS01194\}attP2 }\end{array}$ & BDSC & BDSC: 34715 \\
\hline Atg4bPo997 & $\begin{array}{l}y[1] w\left[{ }^{\star}\right] ; \\
P\{w[+m C]=\operatorname{lac} W\} \operatorname{Atg} 4 b[P 0997]\end{array}$ & BDSC & BDSC: 36340 \\
\hline UAS-bchs-HA & $y[1] w\left[^{\star}\right] ; P\{w[+m C]=U A S-b c h s . H A\} 32$ & BDSC & BDSC: 51636 \\
\hline
\end{tabular}

Abbreviation: BDSC (Bloomington Drosophila Stock Center) 